\title{
EMU DEEPENING AND SOVEREIGN DEBT SPREADS: USING POLITICAL SPACE TO ACHIEVE POLICY SPACE
}

BANCODEESPAÑA

Eurosistema

Documentos de Trabajo

N. ${ }^{\circ} 2103$

Iván Kataryniuk, Víctor Mora-Bajén and Javier J. Pérez 
EMU DEEPENING AND SOVEREIGN DEBT SPREADS: USING

POLITICAL SPACE TO ACHIEVE POLICY SPACE 
EMU DEEPENING AND SOVEREIGN DEBT SPREADS: USING

POLITICAL SPACE TO ACHIEVE POLICY SPACE ${ }^{(*)}$

Iván Kataryniuk, Víctor Mora-Bajén and Javier J. Pérez

BANCO DE ESPAÑA

(*) We thank Aitor Erce, Pietro Rizza, Dominik Thaler, and seminar participants at a Banco de España seminar for their comments and suggestions. The views expressed in this paper are the authors' and do not necessarily reflect those of the Banco de España or the Eurosystem. We thank Fernando Peinado for his outstanding research assistance.

Documentos de Trabajo. N. ${ }^{\circ} 2103$ 
The Working Paper Series seeks to disseminate original research in economics and finance. All papers have been anonymously refereed. By publishing these papers, the Banco de España aims to contribute to economic analysis and, in particular, to knowledge of the Spanish economy and its international environment.

The opinions and analyses in the Working Paper Series are the responsibility of the authors and, therefore, do not necessarily coincide with those of the Banco de España or the Eurosystem.

The Banco de España disseminates its main reports and most of its publications via the Internet at the following website: http://www.bde.es.

Reproduction for educational and non-commercial purposes is permitted provided that the source is acknowledged.

C BANCO DE ESPAÑA, Madrid, 2021

ISSN: 1579-8666 (on line) 


\begin{abstract}
Sovereign spreads within the European Monetary Union (EMU) arise because markets price-in heterogeneous country fundamentals, but also re-denomination risks, given the incomplete nature of EMU. This creates a permanent risk of financial fragmentation within the area. In this paper we claim that political decisions that signal commitment to safeguarding the adequate functioning of the euro area influence investors' valuations. We focus on decisions conducive to enhancing the institutional framework of the euro area ("EMU deepening"). To test our hypothesis we build a comprehensive narrative of events (decisions) from all documents and press releases issued by the Council of the EU and the European Council during the period January 2010 to March 2020. We categorize the events as dealing with: (i) economic and financial integration; (ii) fiscal policy; (iii) bailouts. With our extremely rich narrative at hand, we conduct event-study regressions with daily data to assess the impact of events on sovereign bond yields and find that indeed decisions on financial integration drive down periphery spreads. Moreover, while decisions on key subjects present a robust effect, this is not the case with prior discussions on those subjects at the Council level. Finally, we show that the impacts arise from reductions in peripheral sovereign spreads, and not by the opposite movement in core countries. We conclude that EU policy-makers have at their disposal signicant "political space" to reduce fragmentation and gain "policy space".
\end{abstract}

Keywords: Euro area, EU integration, sovereign spreads, financial fragmentation.

JEL classification: E44, F36, G14. 


\section{Resumen}

La aparición de diferenciales soberanos dentro de la Unión Económica y Monetaria (UEM) responde a que los mercados incorporan en el precio de la deuda pública los fundamentales heterogéneos de los países, pero también el riesgo de redenominación, dada la naturaleza incompleta de la UEM. Esto crea un riesgo permanente de fragmentación financiera. En este documento afirmamos que las decisiones políticas que señalan compromiso por salvaguardar el funcionamiento adecuado del área del euro influyen en los diferenciales soberanos. Para ello, analizamos las decisiones políticas que conducen a mejorar el marco institucional del área del euro. Con el fin de probar nuestra hipótesis, elaboramos una narrativa que clasifica las discusiones y las decisiones reflejadas en todos los documentos y comunicados de prensa emitidos por el Consejo de la Unión Europea (UE) y por el Consejo Europeo durante el período de enero de 2010 a marzo de 2020. Clasificamos los eventos en: (i) integración económica y financiera; (ii) política fiscal, y (iii) rescates. A partir de esta detallada narrativa, llevamos a cabo un análisis de eventos con datos diarios para evaluar el impacto de las decisiones en los diferenciales soberanos. Los resultados señalan que las decisiones sobre integración financiera reducen los diferenciales en los países periféricos. Además, si bien las decisiones sobre temas clave presentan un efecto robusto, este no es el caso de los debates previos sobre estos temas en el ámbito del Consejo. Finalmente, mostramos que los impactos surgen de reducciones en los diferenciales soberanos periféricos, y no por el movimiento opuesto en los países centrales. Concluimos que los responsables políticos de la UE pueden tomar decisiones dentro del «espacio político», que permiten reducir la fragmentación y ganar «espacio para políticas económicas».

Palabras clave: área del euro, integración de la UE, diferenciales soberanos, fragmentación financiera.

Códigos JEL: E44, F36, G14. 


\section{Introduction}

Political events, public speeches and the ability of policy makers to reach agreements can influence sovereign spreads. This is particularly relevant for the European Monetary Union (EMU), as market perceptions of risk, sometimes beyond what fundamentals would tell (Aizenman et al. (2013), De Grauwe (2011); De Grauwe and Ji (2012); Saka et al. (2015)), are entangled with redenomination risk, the possibility that the euro area would break up and bonds would no longer be denominated in the common currency (Gros (2018)). These considerations were quite evident during the debate to set up a European Recovery Fund in 2020 to combat the consequences of the COVID-19 crisis, when news about its size and characteristics were able to drive down market perceptions of credit risk against a background of rising public debt ratios and a profound economic downturn. ${ }^{1}$

The literature has dealt with the issue of how policy announcements or actions affect financial markets mainly by focusing on some specific, salient events, most notably in the area of monetary policy (Bergman et al. (2019); Rivolta (2014); Ait-Sahalia et al. (2012)) In this paper, we take a broader approach. To study how political decisions on European integration affect the sovereign bond market, we collect all documents and press releases by the Council of the EU and the European Council (in their different forms) over the past decade, and identify within this corpus when political decisions were made. Then, we classify the decisions by theme, distinguishing between decisions that refer to one country in particular (Excessive Deficit Procedures or bailout programs being prominent examples) from those that concern the European Union (or the euro area) as a whole. Thus, we compile a complete narrative of European institution's decisions regarding economic and financial integration, and fiscal polcy decisions. This permits us to study the effects of these decisions on financial markets without the need of determining, ex-ante, what decisions drive sovereign yields.

We employ event study methods to confront our narrative with the dynamics of euro area countries' sovereign bond yields. The hypothesis is that noteworthy decisions reduce the sovereign spread of those countries regarded as riskier with respect to the risk-free equivalent bond. The ulterior motive is that markets are expected to behold the euro area as a true economic integrated area if political decisions in this direction are taken.

The main finding of the paper is that final Council decisions on financial integration drive down sovereign spreads in the periphery. The effect is robust to the inclusion of a complete set

\footnotetext{
${ }^{1}$ See Bank of Spain (2020)).
} 
of liquidity, fiscal, credit ratings and financial controls, and also to the consideration of several pre-identified events and ECB meetings. Moreover, we show that the effects on spreads arise mainly from the reduction in peripheral euro area countries' yields, and not by the opposite movement in core countries. Interestingly, we do not find statistically robust effects for meetings not leading to decisions. Thus, our results suggest that the political will to advance in European integration is a key factor to reduce the risks of financial fragmentation in the euro area. Governments could take advantage of this "political space" and reduce pressure on national yields, thus gaining "policy space".

The rest of the paper is structured as follows. In Section 2 we briefly review the related literature, while in sections 3 and 4 we detail the construction on the narrative and the empirical methodology used in the analysis. In turn, we present the main results of our analysis in Section 5 and some concluding remarks in Section 6.

\section{Brief literature review}

A wealth of the literature has focused over the past decade on the reasons underlying the observed heterogeneity in the dynamics of euro area governments' bond yields. There is a broad literature on how sovereign yields' and spreads' dynamics are determined by fundamental variables linked to debt sustainability, such as debt ratios and potential growth (see, among others, Barrios et al. (2009), Sgherri and Zoli (2009), Von Hagen et al. (2011), Barbosa et al. (2010), Poghosyan (2014), Beirne and Fratzscher (2013), Aizenman et al. (2013), Costantini et al. (2014), Afonso et al. (2015) or Georgoutsos and Migiakis (2018))

Within this broad framework, our paper is connected to a strand of the economic literature that moves one step away from traditional determinants. De Grauwe (2011) and De Grauwe and Ji (2012) claim that market overreaction about the perception of default risk might provoke a self-fulfilling solvency crisis. Some studies, such as Dewachter et al. (2015) quantify that at least $20 \%$ of the spread of periphery countries is not explained by fundamentals. Beirne and Fratzscher (2013) and Von Hagen et al. (2011) report evidence that the increase in spreads during the euro crisis in 2010-2012 took place mainly because of a different evaluation of fundamentals by the market in relation to the period prior to the crisis.

The acknowledgment that spreads might react to economic news and institutional statements has inspired a growing number of economic studies. Mohl and Sondermann (2013) find that news about the situation of banks are one of the main elements explaining the 
increase in the pressure on sovereign yields across the euro area. Similarly, Afonso et al. (2020) focus on fiscal decisions, finding that excessive deficit procedures increase the bond spread significantly. In the context of monetary policy, the literature is even more developed. Focusing on Europe, Altavilla et al. (2014) obtained that ECB announcements on Outright Monetary Transactions (OMT) had a significant repercussion by reducing yields in Spain and Italy, but leaving French and German yields unchanged. Rivolta (2014) report that bond acquisition by the ECB caused a reduction in bond spreads in the Eurozone.

Finally, and closer to this paper, some authors have investigated the effects of news related to the general European governance framework. As an example, Bergman et al. (2019) investigates the effects of a set of decisions of European policymakers on banking CDS and sovereign yields. They find that ECB actions, such as the announcement of the Outright Monetary Transactions programme, lowered spreads, and so did the announcement of fiscal agreements in the Council. However, reforms in the banking system had little to no effect. Beetsma et al. (2013) assert that the presence of more negative news about one of the peripheral countries causes sovereign yields in that country to increment, as well as spillover effects in the rest of the peripheral countries. In line with the last finding, the literature has drilled into how contagion coming from news and announcements spreads across countries in

Figure 1: Evolution of 10 year sovereign yields and public debt (as \% of GDP)

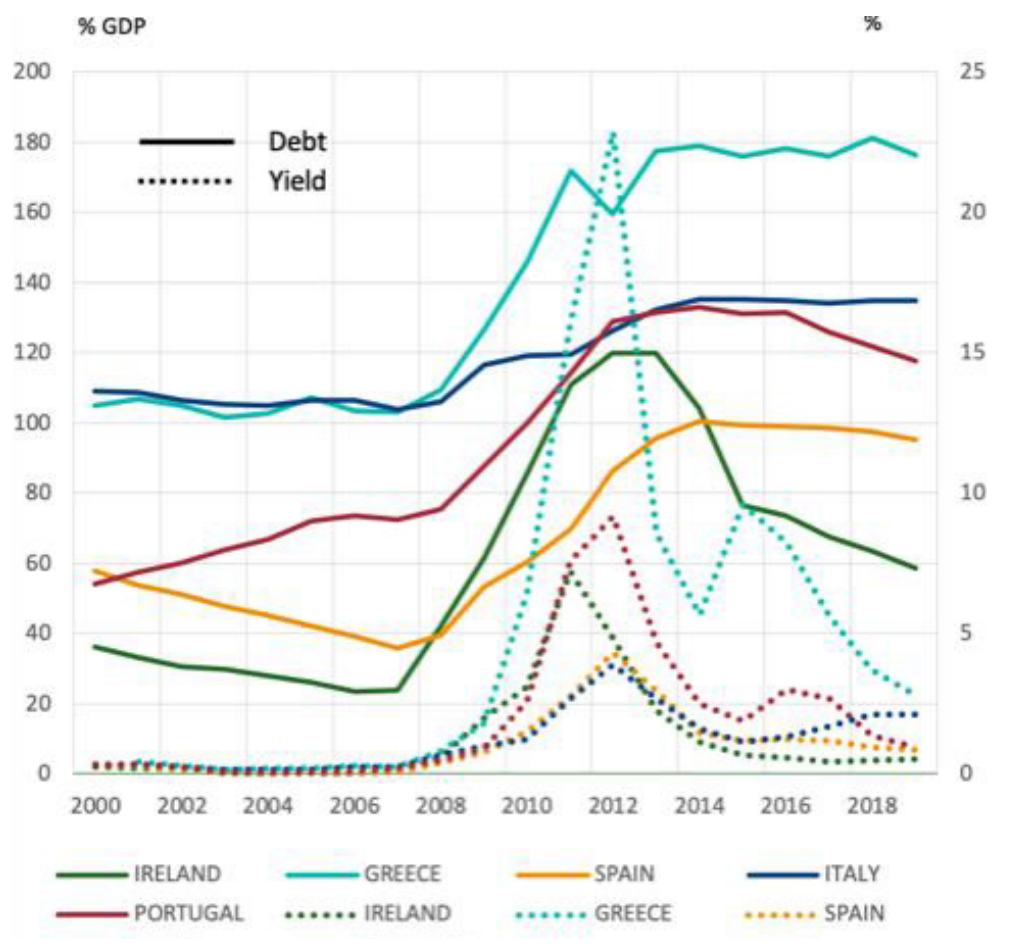

Source: Eurostat. 
the euro area. An example of this is Bahaj (2020). The authors construct a narrative that focuses on national news affecting one particular country and document how they spread to other countries considered as risky.

\section{The narrative dataset: overview}

We build a comprehensive narrative that ranges from January 2010 until March 2020. In this section we give an overview of its main elements. We provide more details in annexes $\mathrm{A}$ and $\mathrm{B}$.

In order to construct the narrative, we compile all ECOFIN, Eurogroup and European Council meetings and press releases that have taken place within this period. These documents are available at the website of the Consilium. The starting date is determined by the lack of (or only partial) availability of documents in the public domain prior to 2010 . In total, we have collected documents from 516 unique meetings in the period that is being assessed. In order to check that we do not miss any significant meeting or press release, we read throughout all the compiled documents and double-checked all references made within these to previous meetings.

Figure 2: Discussion of topics of relevance over time.

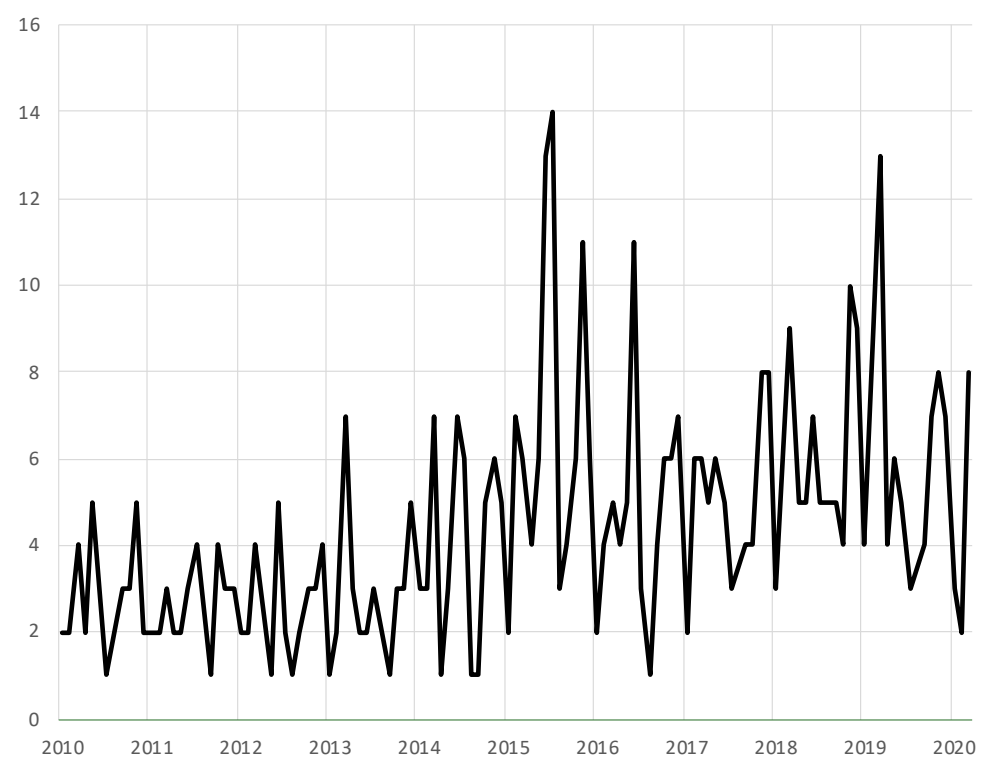

Note: the figure dates the 516 meetings identified from the documents. 
The narrative includes each meeting or press release as many times as they deal with discussions including a specific policy topic of relevance for our study. In total, the 516 meetings reference 689 discussions. Should there not be any topic of significance, the meeting will also have one entry in the dataset. Figure 2 depicts how these events are distributed over time. There is a peak in 2015 that corresponds to the Greek crisis in the context of the third bailout programme and the referendum that took place. In addition, there is another concentration of meetings in the Summer of 2016, linked to Brexit-related discussions. There is a third spike in 2019, that shows an increase in the political willingness to discuss euro area deepening.

We classify all the discussions (either ending up in a decision or not) in two main groups. The first group includes events that affect one country. Within this group, we distinguish two categories: (i) crisis management: this category involves mainly bailout programs and their revisions; (ii) all topics about national fiscal policy procedures or instruments, most notably excessive deficit procedures, either opened or closed. The second group includes events that affect all countries. Within this latter class we include topics related to: (i) common fiscal policies, like those dealing with common fiscal resources or aimed at enhancing budgetary discipline from a cross-country perspective and/or at a supranational level, such as the approval of the so-called Six-Pack and Two-Pack, or the debates around the Budgetary Instrument for Convergence and Competitiveness (BICC); (ii) financial integration topics, like, for instance, the creation of new institutions, such as the European Stability Mechanism (ESM) or the Single Supervisory Mechanism (SSM); Capital Markets Union (CMU) project, as they have tended to be smaller in scope than the ones included in the previous category and they are clustered within specific years.

Once we have a complete classification of discussions and country-specific decisions, we move towards discerning whether a final decision has been taken on a given topic of interest related to the euro area as a whole.

The usual procedure to approve any law at the supranational level commences with a proposal coming from the European Commission (Figure 3). On the basis of the proposal, the Council debates and adopts a stance in order to negotiate with Parliament. In these negotiations, both institutions agree on the amendments, and the legislation is finally adopted. We consider this moment as the "decision" point. Sometimes, the agreed legislation takes some time until it enters into force, with some delays related, for example, to approval by national parliaments. Let the approval procedure of the so-called "Two-Pack" serve as an 
Figure 3: Simplified decision path at the European level

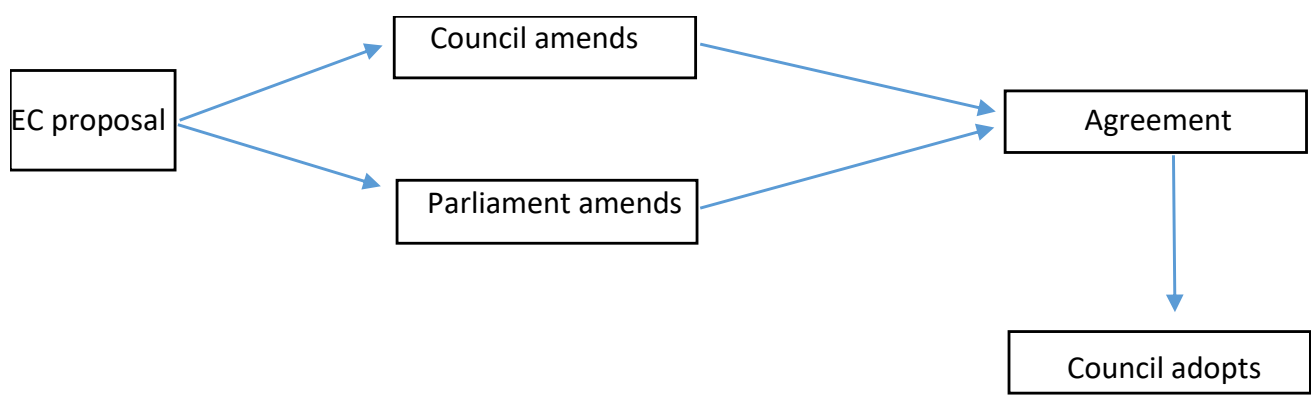

illustrative example. On November 23, 2011, the European Commission launched its initiatives for a second package of proposals for the strengthening of economic governance. The Council took note on November 30 and that marks the initial step of the approval procedure in our narrative. The regulation was debated within the Council, that subsequently agreed on a final stance for negotiations with the Parliament on November 13, 2012. On February 20, 2013, the Council and the Parliament reached an agreement. Finally, on May 13, 2013, the Council finally adopted the Two-Pack. This latter date is considered as the final decision in our narrative.

Sometimes, we find that a European forum discusses several topics, but only decides on a few ones. For example, in the Eurogroup meeting of 4 December 2018 Ministers discussed about BICC, the European Deposit Insurance Scheme (EDIS), the ESM reform and the backstop for the Single Resolution Fund (SRF), but only took decisions on the last two. As a result, our database will register four discussions but only two decisions in that day.

We only include as decisions the final adoption of a specific legislative piece, and therefore we do not register as decisions roadmap documents, such as the "Five Presidents Report". This comes as a consequence of the fact that these documents tend to be very general and do not change any specific policy. For decisions that do not have to go through the Parliament (for instance, the establishment of the European Financial Stability Facility, EFSF, and the European Financial Stabilisation Mechanism, EFSM), the same consideration about the final decision holds. Another example is the ESM approval procedure, which covers a long stretch of time, throughout which the Council took intermediate decisions about partial features of the instrument, but only the final ESM Treaty was subjected to national parliament's approval. In this case, we included the decisions changing relevant parts of the policy framework. 
For our analysis, it is important to discern between topics leading to decisions from those that were discussed but did not lead to decisions. For example, there are 16 discussions in our narrative about EDIS, with no decisions taken at all. Similarly, we register 13 discussions about the treatment of Non Performing Loans, until a decision was made on 9 April 2019. As can be seen in figures 4 and 5 , decisions are clustered around some years. That is the

\section{Figure 4: Distribution over time of decisions}

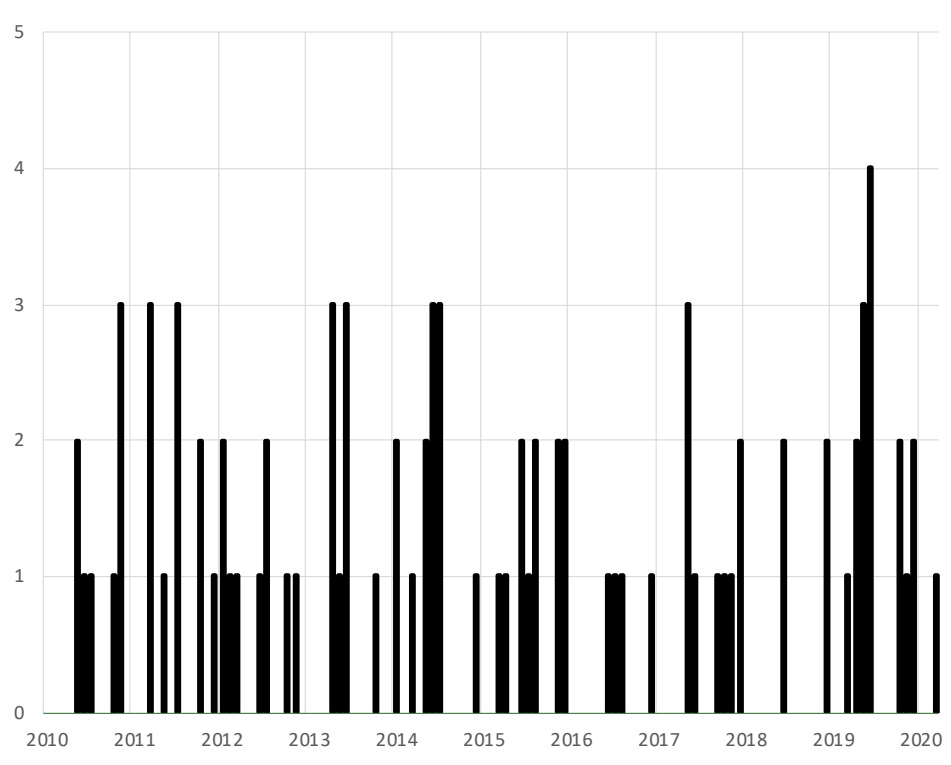

Note: The figure shows the distribution over time of the 91 decisions identified.

Figure 5: Distribution over time of decisions by topic

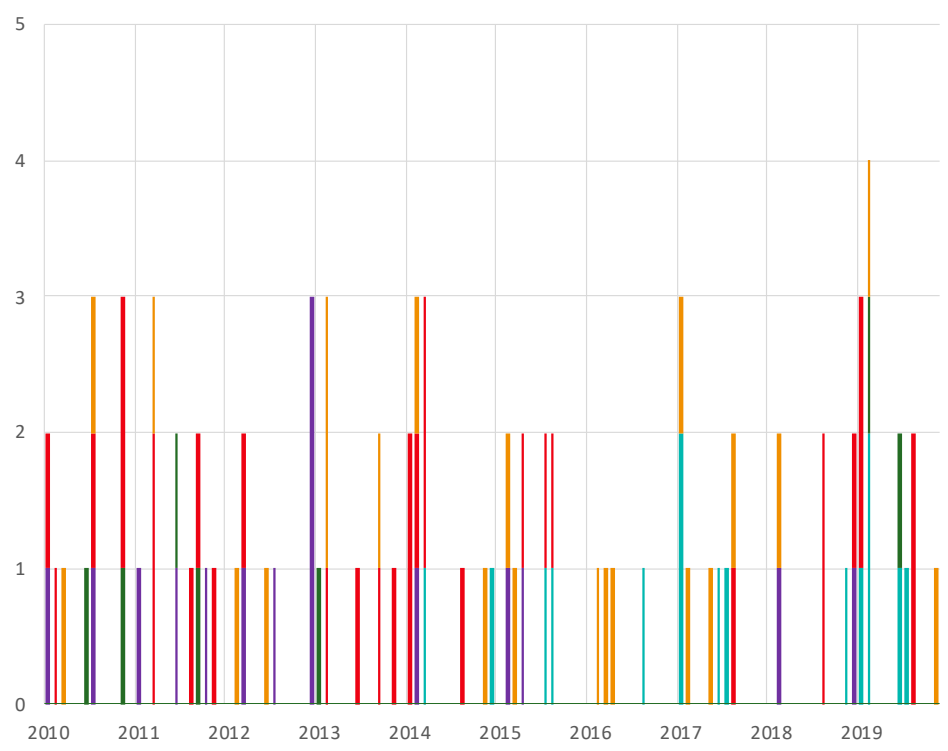

Note: The figure shows the distribution by topic of the 91 decisions identified. 
case for bailout decisions, in the period between 2011 and 2015, or decisions regarding the Capital Markets Union project, from 2015 to 2019. The complete narrative of decisions, together with a summary table, is provided in Annex A.

In total, we categorize 91 decisions, among which 37 do refer to individual events (either of a fiscal nature or of a crisis management nature), and 54 on economic integration. As stated before, we further classify the latter 54 decisions in three groups: (i) 32 decisions are associated with financial integration (see Table 1); (ii) 7 are decisions related to fiscal policy and fiscal coordination (see Table 2); (iii) 15 decisions related to the CMU (see Table B.3). A complete breakdown of events is provided in Annex B.

\section{Empirical exercise: methodology}

We use a panel data regression with fixed effects to link daily changes in euro area countries' 10 year sovereign yield spreads with narrative events. We focus on the so-called peripheral euro area countries, namely Italy, Spain, Portugal, Ireland and Greece. In this model we introduce dummies for decision dates and the coefficients will capture the effect of being in such a day with respect to any other day. Thus, these dummies would capture the effect of a certain decision that has been taken on a certain date, under the assumption that no other important economic or political events have taken place within the same day, which would render the effect impossible to disentangle. Given that a meeting might provoke uncertainty or ex-ante expectations and that the consequences of any decision might be persistent or delayed, we use a window of three days prior and following the decision in order to evaluate the impact on sovereign yields.

We estimate the following equation for a panel of data pertaining to euro area countries:

$$
\triangle y_{i t}=\alpha+\sum_{t=-3}^{t=+3} \beta_{t} * \text { decision }_{t}+\gamma * X_{i t}+\varepsilon_{i t}
$$

Where subscript i represents a country and subscript t refers to each working day when markets open. $y$ captures a change in the spread with respect to the risk-free OIS yield (or the Bund yield) from one day to the next. The main variable of interest is decision. We only include a dummy for the decisions affecting all countries at the same time. Notice that some decision are made in the same date, and they are considered as a single event. As explained later, we will use country-specific decisions as controls. For decisions that fall into a non-working day, we move all yields and financial controls in subsequent dates as many 
Table 1: Decisions related to financial integration (finance and banking)

\begin{tabular}{|c|c|}
\hline Date & Main decisions \\
\hline $5 / 9 / 10$ & EFSM \\
\hline $6 / 7 / 10$ & EFSF \\
\hline $11 / 17 / 10$ & ESMA, ESRB, EBA and EIOPA \\
\hline $3 / 11 / 11$ & EFSF/ESM ceiling EUR 440 bn \\
\hline $3 / 25 / 11$ & Article 136/Euro Plus Pact \\
\hline $7 / 11 / 11$ & ESM first version \\
\hline $7 / 21 / 11$ & Increase flexibility EFSF/ESM \\
\hline $12 / 9 / 11$ & EFSF/ESM ceiling EUR 500 bn \\
\hline $1 / 30 / 12$ & ESM Treaty ready/Fiscal Compact \\
\hline $3 / 30 / 12$ & EFSF/ESM ceiling EUR 700 bn \\
\hline $7 / 9 / 12$ & EFSF/ESM as efficient conduct of market operations \\
\hline $6 / 20 / 13$ & Main feautres recapitalisation instrument ESM \\
\hline $10 / 15 / 13$ & SSM \\
\hline $1 / 28 / 14$ & Single mortgage credit \\
\hline $3 / 3 / 14$ & National Deposit Guarantee Schemes (DGS) \\
\hline $5 / 6 / 14$ & Harmonisation national rules on bank recovery and resolution \\
\hline $5 / 21 / 14$ & Bank Resolution Fund \\
\hline $6 / 10 / 14$ & Operationalisation framework ESM recapitalisation instrument \\
\hline $7 / 8 / 14$ & ToR capital shortfalls and budget sharing \\
\hline $7 / 14 / 14$ & SRM \\
\hline $12 / 19 / 14$ & Contributions to SRB \\
\hline $8 / 4 / 15$ & EFSM shield non-euro area countries \\
\hline $11 / 30 / 15$ & SRM confirmation (January 1, 2016) \\
\hline $12 / 8 / 15$ & Public bridge financing for SRF \\
\hline $12 / 7 / 17$ & Bank creditor hierarchy and IFRS accounting standards \\
\hline $12 / 4 / 18$ & Operationalisation common backstop SRF \\
\hline $12 / 4 / 18$ & ESM reform \\
\hline $4 / 9 / 19$ & Non-Performing Loans (NPL) framework \\
\hline $5 / 14 / 19$ & Banking risk reduction package \\
\hline $5 / 14 / 19$ & Over-the-counter (OTC) framework \\
\hline $12 / 2 / 19$ & Revision banking supervision framework \\
\hline $12 / 4 / 19$ & ESM backstop \\
\hline
\end{tabular}

Table 2: Decisions related to fiscal policy

\begin{tabular}{ll} 
Fecha & Decision \\
\hline $10 / 21 / 10$ & European Semester \\
\hline $3 / 25 / 11$ & Euro Plus Pact \\
\hline $10 / 4 / 11$ & Six-Pack \\
\hline $1 / 30 / 12$ & Fiscal Compact \\
\hline $5 / 13 / 13$ & Two-pack \\
\hline $6 / 13 / 19$ & Main features BICC \\
\hline $10 / 9 / 19$ & Final approval BICC \\
\hline
\end{tabular}


days as necessary to assign the next working day data point to the decision date. The same procedure applies to the next dates within the window period. Event t-3 marks all dates that are 3 days prior to the event, t-2 all of those that are 2 days prior to the event, and so it goes until $\mathrm{t}+3$, which collects all dates 3 days posterior to the event.

Our coefficients of interest are $\beta_{0}$ and $\beta_{1}$ because of a potential lagged effect that may arise from the fact that typically, when meetings are held in working days, they last until markets are already closed.

As controls, we include the spread between the bid and the ask price of the ten year sovereign bond, as a proxy for liquidity in the market, the first difference of the EONIA rate, the VIX and the first difference in stock exchange returns of a given country. Moreover, we control for changes in credit ratings, by including a dummy which takes value of 1 if there has been a downgrade of the national sovereign rating, - 1 if there has been an upgrade and 0 otherwise. We control for monetary policy with the use of several variables. First, we include a dummy in the date of each ECB meeting, which takes a value of 1 if there has been a monetary policy meeting at that date and 0 otherwise. However, this would capture only average effects, and there are some specific events that might need a more direct treatment. As a result, we include a period dummy that takes value of 1 between the date of the "whatever it takes" statement (July 26, 2012) and the day that the ECB announced the extension of the Asset Purchase Programme (APP) to sovereign bonds (January 22, 2015). From that date up until the current date, we include a dummy that take value of 1 to account for the APP period. Definition and sources of all variables can be found in Table 4 .

We include two other variables from our narrative to control for political events. One of them is a dummy taking value of 1 when there are positive fiscal decisions (e.g. and EDP in closed) and -1 when there are negative decisions (e.g. an EDP is open), following Afonso et al. (2020) (see Annex A for details). The other dummy takes a value of 1 when there are decisions related to bailouts and 0 otherwise (see Annex B for details). A set of the most significant European Commission proposals for European integration are also incorporated.

Finally, we also include day-of-the-week dummies in all regressions given that the days in which meetings are usually held are not random: it is more likely that $t=0$ corresponds to a Monday or a Tuesday. Moreover, in some specifications, we include a lag of the dependent variable, to correct for the possible time dependence of our variable of interest.

Under the usual assumptions that the errors are not correlated with the explanatory variables in every $t$ and the expected value of the residuals is zero, each of the estimated 
coefficients will be equal to the population coefficients. Thus, each of the betas will capture the true abnormal return in each day within the assessment window. By aggregating the effect of the betas, the statistical inference will take into account the mean yield and mean variances across countries in each day, as Pynnönen (2005) suggests for event windows that are over-lapping across countries. However, for testing purposes, the assumption that errors are not serially correlated or cross-correlated is unlikely to hold. Sovereign yields' changes are expected to depend on their behavior in previous days. Furthermore, country i yields will also be subject to how other country j yields behave, as others authors have proven (Bahaj (2020); Bhanot et al. (2012); Mink and De Haan (2013);Missio and Watzka (2011). Therefore, the errors are corrected for cross-correlation and autocorrelation.

Sovereign spreads may influence the legislative process. For example, an increase around a Council meeting might put pressure to approve one legislative package. Moreover, some topics might be introduced (and approved) in the agenda because of market pressure. This would create a problem of endogeneity in our estimations, and would bias our results. In order to check if this is the case, we run standard predictability tests by reverting the order of causality, by running regressions of the following type:

$$
\text { decision }_{t}=\alpha_{0}+\alpha_{1} \triangle y_{i t-1}+\alpha_{2} \triangle y_{i t-2}+\gamma X_{i t}+\varepsilon_{i t}
$$

and

$$
\text { decision }_{t+1}=\alpha_{0}+\alpha_{1} \triangle y_{i t}+\alpha_{2} \triangle y_{i t-1}+\gamma X_{i t}+\varepsilon_{i t+1}
$$

for the considered euro area countries (Greece, Ireland, Italy, Portugal and Spain). The results of this exercise are shown in Table 3. The table shows results using OLS for the estimation (a logit regression approach yielded similar outcomes). For both $t=0$ (first column) and $t=1$ (second column) there is no evidence of spreads leading to a particular decision in a given date.

Table 3: Predictability-tests: null hypothesis that the spread variable does not Granger-cause the narrative variable.

\begin{tabular}{lcc}
\hline & Equation (2) & Equation (3) \\
\hline F test & 0.738 & 0.818 \\
p-value for F test: & 0.390 & 0.366 \\
\hline
\end{tabular}

Note: The table shows the results of a F-test, to determine if the coefficients of the regression of the spreads on the date of the events are jointly different from zero. The dependent variable are the financial and fiscal decisions affecting all countries at the same time. Standard errors are robust to heteroskedasticity and autocorrelation. 


\section{Results}

We show the main results of our analysis in tables 5,6 , and 7 .

First, in Table 5 we show the results of the regressions when the dependent variable is the 10-year sovereign yield spread with respect to the OIS risk-free rate. In column 1 we present the results for the case in which we include only general controls. As can be seen, political decisions play a role in shaping the behavior of bond yields in peripheral countries: the spreads decrease by over 4 basis points, on average, in each following day to any relevant decision taken by the European institutions. For the sake of the illustration, we show the coefficients of the event variables (within two-standard deviations bands) in figure 6. In addition, some anticipation effect is visible in prior days, whereby an increase in the spread may be anticipating some uncertainty around the outcome of the policy discussion, given the traditional hurdle around decisions in the European Union. Finally, there is no reversion of the effects in the following days after the event. One could argue that this could be expected, as the events considered change the structural nature of economic integration in the European Union, and, subsequently, change the expected path of future repayments of the debt. Nonetheless, this argumentation is merely speculative, and we leave further inquiries into this issue for further research.

Our key result is robust to the inclusion of additional control variables in column 2 , the exclusion of Greece (column 3) and the addition of up to 4 lags of the dependent variable (for the sake of brevity, we only display column 4 with one lag, since the results with more lags are very similar). The reduction for the case of Spain halves but stays significant (column 5), whereas there seems to be no effect for the Italian spread (column 6). In column 7 we set Spain and Italy together, where the latter seems to be rendering the coefficient of $t+1$ insignificant. Column 8 shows the outcome when only Ireland and Portugal are considered, while column 9 shows the results for Greece only. The relatively smaller countries experience a larger and more significant reduction in spreads after European decisions in comparison to the two larger Southern countries. When we stick only to financial integration events, the coefficient stays significant but the magnitude gets diminished (column 10). This outcome is consistent with Gerlach et al. (2010), who find that, apart from the effect of fundamentals, up to one percentage point of the increase in the spreads in the Eurozone during the financial crisis can be explained by global banking risks. Adding decisions related to Capital Markets Union (column 11) also drives the coefficient down.

Control variables display the expected signs in most cases. We find a positive effect of the VIX on spreads, even though it is only statistically significant in the regressions that include 
Figure 6: Estimated impact of narrative decisions on 10-year spreads with respect to the OIS. Peripheral countries. General control variables.

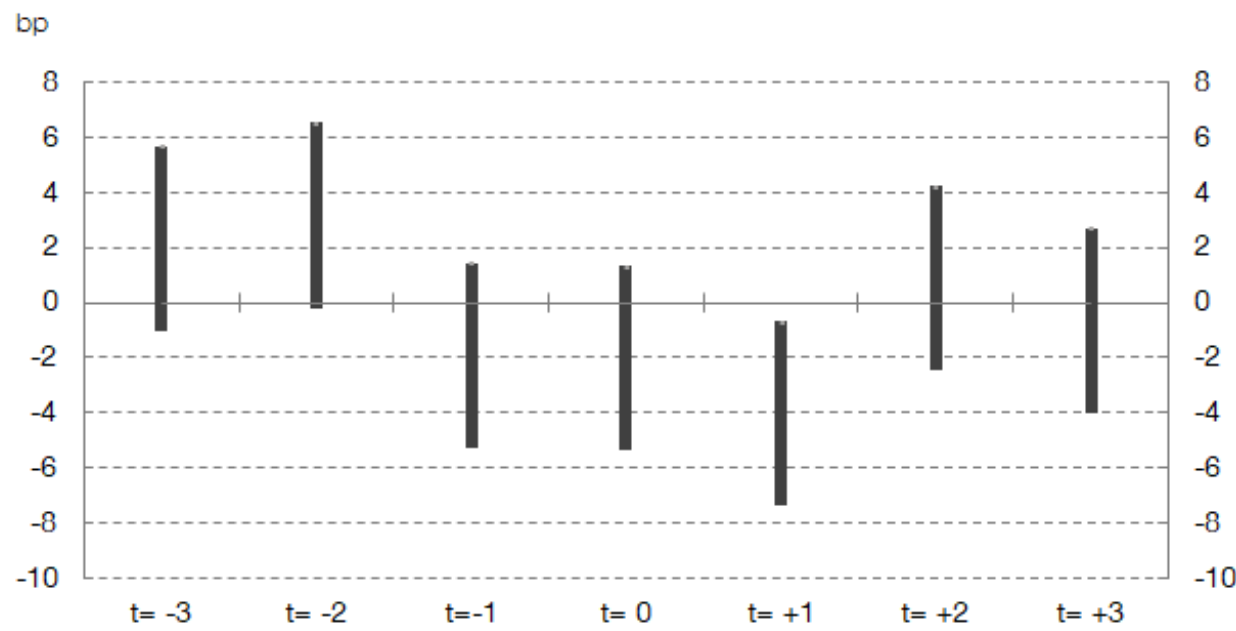

Note: The figure shows the impact on average and two standard deviations. The specification corresponds to the one in column 1 of Table 5 .

Greece. The stock market index is negative and significant in all regressions. The EONIA and the change in credit ratings are not significant in our regression. The result on the bid-ask spread merits some attention, as it has a negative coefficient in the baseline. Investigating further, this effect only appears either in regressions with Greece, and, in particular, when taking into account the year 2012. Given that this is the year of the Greek debt restructuring, the liquidity crunch coincided with a moment when spreads were detached from fundamentals. Regarding the control variables taken from our narrative dataset, fiscal and bailout decisions do not show a statistically significant effect. Notwithstanding, regarding the latter, there is, as expected, a significant negative sign of more than 6 basis points in the regression that involves Ireland and Portugal. Similarly to Krishnamurthy et al. (2018), we find a very significant effect of monetary policy decisions on spreads, in particular, related to the "whatever it takes" date. This effect weathers out throughout the whole period but it does not vanish completely. From our set of important Commission proposals, the SSM, the "Two-pack" and the BICC seem to have a remarkable and significant effect in some of our specifications, which is consistent with the results in Bergman et al. (2019). The publication of the "Five Presidents Report" also had a remarkable impact in bringing confidence to the market. 
Second, in Table 6 we show the results of the regressions when the dependent variable is the 10-year sovereign yield spread with respect to the equivalent German instrument. Results are qualitatively very close to the OIS-referenced ones. Interestingly, the anticipation effect whereby spreads increase prior to the decision gets somewhat amplified in this case, most likely reflecting that the uncertainty about a positive outcome leads investors to move to the German reference, a traditional safe haven for euro paper.

Finally, in Table 7 we present a placebo exercise. One potential source of concern could be that in the policy-making process in Europe previous steps are more likely to lead to market reactions. In order to test that this does not happen, we center our daily window around the meeting taking place prior to each decision (which is labeled as a 0 in the original regression). Typically, the previous document or meeting announces an agreement with the European Parliament or gives away details of an agreement or the reconciliation of different views in the Council ${ }^{2}$. Looking at the outputs, the previous positive reaction of the markets on day $t+1$ does not appear, corroborating that our choice of the date of the decision is robust.

Our results are robust to the choice of the length of the window for the event analysis. Anticipation effects might take place in a framework where European meetings are scheduled well in advance. Moreover, topics are usually discussed in previous meetings, information is usually leaked in advance and institutions tend to signal proximity to the agreement. As explained, this was the reasoning leading us to choose a window of three days centered around the event day. However, there is a trade-off, to the extent that a longer window could lead to biased estimates, since it could be picking up the effect of any other relevant event that occurs within the window. Thus, we run the same regression tightening the event period to one day prior and following the event. Our main result, that is, the finding of a reduction in spreads the day after the decision, remains significant and very similar in magnitude, which implies that the outcome is robust to different windows.(Tables C.1 and Table C.2 in the appendix).

In Table 8 we present an additional robustness piece. Given that the spreads we focus on in our analysis are the difference between the yields of bonds considered more risky with respect to safer references, one may wonder if the results reflect genuine reductions in peripheral sovereign yields, or the opposite movement in core countries. In the table we

\footnotetext{
${ }^{2}$ Notice that the number of decisions change slightly, as in some cases previous discussion refers to a date outside our sample period and in other cases some decisions that are taken in packages (like those referring to $\mathrm{CMU}$ ) are discussed separately in the previous instances. See more details in the notes of Table 7
} 
show regressions for a pool that takes into account additional countries: the peripheral ones (Italy, Spain, Portugal, Ireland and Greece) and the so-called "core" ones (Germany, France and The Netherlands). The outcomes of our empirical explorations are clear: the results are genuinely led by the reduction in riskier countries' yields.

\section{Conclusions}

We have investigated how European policy-making institutions have an influence on the behaviour of sovereign yields in the euro area. We build on previous research that shows the importance of news and institutional statements on euro area sovereign bond yields. We innovate by creating a whole narrative that compiles all the meetings and press releases published by the main relevant policy-making European institutions between 2010 and 2020 . This allows us to remove the subjective ex-ante judgment as to what decisions are more important and should be included. Moreover, the collected database opens new research paths in the area of text analysis. We find that the day after a major political decision is made at the European level, the spread of those countries considered to be more risky gets reduced. Thus, our paper suggests that EU policymakers enjoy ample political space that could be used to reduce (risks of) financial fragmentation, insofar as this space is used to deepen the economic and monetary union. 
Table 4: Variable definition and sources

Spreads

Decision $\mathrm{t}=\mathrm{n}$

BIDASKDIEZ

EONIA

VIX

ECB

STOCK

MOODY

DEFICIT

BAILOUT

BAILOU

WIT_period

APP

APP period

Two pack

SSM

BICC

SRM

EDIS

EMF

ER5
10 (or 5) year spread with Bund, or spread with respect to the OIS

Dummy $=1$ when a decision on integration (for all countries) is taken, window $=\mathrm{n}$ days

Bid-ask spread of the 10-year bond for each country

Change in the EONIA rate

Dummy $=1$ when an ECB meeting is held

Increase in the country stock index

Dummy $=1$ when there is a downorade in ratings, -1 when there is an uporade

Dummy=1 when a fiscal decision involves a positive development in one country (closing of EDP), $=-1$ when is negative (open of EDP)

Dummy for crisis management decisions involving one country. Dummy $=1$ when a bailout programme is approved

Dummy $=1$ in $7 / 26 / 2012$, the date of Draghi's "Whatever it takes" speech

Dummy $=1$ from WIT start to the APP start

Dummy $=1$ from $1 / 22 / 2015$, when the Asset Purchase Programme of the ECB started Dinny=1 fom the start of the APP

Dummy $=1$ the date of the two pack proposal of the European Commission

Dummy $=1$ the date of the SSM proposal of the European Commission

Dummy $=1$ the date of the BICC proposal of the European Commission

Dummy $=1$ the date of the SRM proposal of the European Commission

Dummy $=1$ the date of the EDIS proposal of the European Commission

Dummy $=1$ the date of the EMF proposal of the European Commission

Dummy $=1$ the date of the PR5 proposal of the European Commission
FAME Information Services See text

FAME Information Services Datastream Datastream ECB

$$
\begin{gathered}
\text { Datastream } \\
\text { Moody's }
\end{gathered}
$$

Moody's

See main text

See main text

Own elaboration

Own elaboration

Own elaboration

Own elaboration

Own elaboration

Own elaboration

Own elaboration

Own elaboration

Own elaboration

Own elaboration

Own elaboration 
Table 5: Results using as dependent variable the 10-year spread with respect to the OIS risk-free reference.

\begin{tabular}{|c|c|c|c|c|c|c|c|c|c|c|c|}
\hline VARIABLES & (1) & (2) & (3) & (4) & (5) & (6) & (7) & (8) & (9) & (10) & (11) \\
\hline \multirow[t]{2}{*}{ Decision $t-3$} & 2.292 & 1.999 & $2.448^{* *}$ & 2.160 & $3.305^{* * *}$ & $3.537^{* * *}$ & $3.399^{* * *}$ & 0.819 & 0.555 & $3.290 *$ & 1.465 \\
\hline & $(1.683)$ & (1.623) & $(1.146)$ & $(1.643)$ & $(1.268)$ & $(1.300)$ & $(1.204)$ & $(1.557)$ & $(2.340)$ & $(1.774)$ & $(1.415)$ \\
\hline \multirow[t]{2}{*}{ Decision t-2 } & $3.156^{*}$ & $3.119^{*}$ & 1.223 & $3.538^{* *}$ & 1.657 & 0.365 & 1.121 & 0.961 & $5.414^{* *}$ & $4.547^{* *}$ & $2.415^{*}$ \\
\hline & $(1.692)$ & (1.633) & $(1.150)$ & $(1.650)$ & $(1.273)$ & (1.307) & (1.209) & $(1.560)$ & $(2.352)$ & $(1.782)$ & $(1.417)$ \\
\hline \multirow[t]{2}{*}{ Decision $t-1$} & -1.910 & -1.527 & -0.840 & -1.322 & -1.272 & -0.181 & -0.523 & -2.081 & -2.729 & -1.706 & -0.220 \\
\hline & (1.669) & (1.635) & $(1.151)$ & (1.681) & $(1.294)$ & $(1.324)$ & $(1.226)$ & $(1.585)$ & $(2.392)$ & $(1.813)$ & (1.438) \\
\hline \multirow[t]{2}{*}{ Decision $t$} & -2.002 & 1.821 & $2.496^{* *}$ & 1.656 & 1.511 & 1.442 & 1.506 & $3.226^{* *}$ & 0.830 & 1.820 & 1.818 \\
\hline & $(1.667)$ & (1.631) & $(1.148)$ & $(1.653)$ & $(1.269)$ & $(1.306)$ & $(1.206)$ & $(1.558)$ & $(2.352)$ & $(1.778)$ & (1.418) \\
\hline Decision $t+1$ & $\begin{array}{l}-4.038 * * \\
(1667)\end{array}$ & $-4.378^{* * *}$ & $-3.122^{* * *}$ & $-4.386 * * *$ & $-2.241^{*}$ & $\begin{array}{l}-1.312 \\
(1.293)\end{array}$ & $\begin{array}{l}-1.762 \\
(1.197)\end{array}$ & $-5.436^{* * * *}$ & $\begin{array}{l}-6.330 * * * \\
(2335)\end{array}$ & $\begin{array}{l}-3.035^{*} \\
(1.785)\end{array}$ & $\begin{array}{l}-2.945 * * \\
(1.409)\end{array}$ \\
\hline \multirow{2}{*}{ Decision $t+2$} & 0.886 & 0.513 & 0.00773 & 0.105 & $\begin{array}{l}1.200) \\
-0.519\end{array}$ & $\begin{array}{l}1.290) \\
-0.203\end{array}$ & $\begin{array}{l}1.197) \\
-0.462\end{array}$ & 1.787 & 0.628 & 0.766 & $\begin{array}{l}1.409) \\
-0.521\end{array}$ \\
\hline & $\begin{array}{l}0.000 \\
(1.666)\end{array}$ & $\begin{array}{l}0.010 \\
(1.608)\end{array}$ & $(1.133)$ & $(1.630)$ & $\begin{array}{l}-0.019 \\
(1.253)\end{array}$ & $\begin{array}{l}-0.285) \\
(1.285)\end{array}$ & $\begin{array}{l}-0.402 \\
(1.189)\end{array}$ & $(1.536)$ & $\begin{array}{l}0.020 \\
(2.319)\end{array}$ & $(1.781)$ & $(1.405)$ \\
\hline Decision $\mathrm{t}+3$ & $\begin{array}{l}-0.685 \\
(1.683)\end{array}$ & $\begin{array}{l}-0.529 \\
(1.622)\end{array}$ & $\begin{array}{l}-0.362 \\
(1.140)\end{array}$ & $\begin{array}{l}-0.613 \\
(1.643)\end{array}$ & $\begin{array}{c}0.362 \\
(1.252)\end{array}$ & $\begin{array}{l}-0.574 \\
(1.306)\end{array}$ & $\begin{array}{r}-0.0198 \\
(1.194)\end{array}$ & $\begin{array}{l}-0.551 \\
(1.558)\end{array}$ & $\begin{array}{l}-0.943 \\
(2.347)\end{array}$ & $\begin{array}{l}-1.169 \\
(1.798)\end{array}$ & $\begin{array}{l}-0.990 \\
(1.414)\end{array}$ \\
\hline BIDASK & $\begin{array}{c}-2.688^{* * *} \\
(0.669)\end{array}$ & $\begin{array}{l}-2.857^{* * * *} \\
(0.660)\end{array}$ & 0.678 & $-2.920^{* * *}$ & 4.998 & 2.005 & 3.044 & -0.542 & $-3.498^{* * *}$ & $-3.069^{* * * *}$ & $-2.975^{* * *}$ \\
\hline \multirow{2}{*}{$\triangle$ EONIA } & $\begin{array}{c}(0.669) \\
-1.180\end{array}$ & $\begin{array}{l}(0.660) \\
-0.983\end{array}$ & $\begin{array}{c}(1.022) \\
0.531\end{array}$ & $\begin{array}{l}(0.678) \\
-1.665\end{array}$ & $\begin{array}{l}(8.480) \\
-2.005\end{array}$ & $\begin{array}{l}(14.79) \\
-2.994\end{array}$ & $\begin{array}{l}(8.823) \\
-2.588\end{array}$ & $\begin{array}{l}(1.104) \\
3.582\end{array}$ & & $\begin{array}{l}(0.667) \\
0.0472\end{array}$ & $\begin{array}{l}(0.667) \\
-1.623\end{array}$ \\
\hline & $(2.970)$ & $(2.882)$ & $(2.030)$ & $(2.908)$ & $(2.290)$ & $(2.351)$ & $(2.174)$ & $(2.771)$ & $(4.102)$ & $(2.912)$ & $\begin{array}{l}-1.020 \\
(2.904)\end{array}$ \\
\hline \multirow[t]{2}{*}{ VIX } & $0.217^{* * *}$ & $0.181^{* * * *}$ & $\begin{array}{l}0.0111 \\
0.01200\end{array}$ & $0.196^{* * *}$ & -0.0381 & -0.0354 & -0.0276 & -0.0167 & $0.309^{* * * *}$ & $0.206^{* * *}$ & $0.193^{* * *}$ \\
\hline & $(0.0356)$ & $(0.0418)$ & $(0.0300)$ & $(0.0435)$ & $(0.0309)$ & $(0.0308)$ & $(0.0290)$ & $(0.0393)$ & $(0.0634)$ & $(0.0434)$ & $(0.0435)$ \\
\hline $\mathrm{ECB}$ & $\begin{array}{c}1.253 \\
(1.002)\end{array}$ & $\begin{array}{c}0.204 \\
(1.046)\end{array}$ & $\begin{array}{l}-1.115 \\
(0.732)\end{array}$ & $\begin{array}{c}0.135 \\
(1.061)\end{array}$ & $\begin{array}{l}-1.446^{*} \\
(0.814)\end{array}$ & $\begin{array}{c}-2.186 * * * \\
(0.832)\end{array}$ & $\begin{array}{l}-1.871^{* *} \\
(077 ?)\end{array}$ & $\begin{array}{l}-0.192 \\
(1009)\end{array}$ & $\begin{array}{l}1.848 \\
(1513\end{array}$ & 0.135 & 0.165 \\
\hline \multirow[t]{2}{*}{$\triangle \mathrm{STOCK}$} & $-0.0235^{* * *}$ & $-0.0208^{* * *}$ & $-0.0164^{* * *}$ & $-0.0205^{* * *}$ & $-0.0256^{* * *}$ & $\begin{aligned} & (0.01292) * * *\end{aligned}$ & $-0.0148^{* * *}$ & $-0.0655^{* * * *}$ & $-0.0723^{* * *}$ & $-0.0213^{* * * *}$ & $-0.0212^{* * *}$ \\
\hline & $(0.000826)$ & $(0.000834)$ & $(0.000569)$ & $(0.000841)$ & $(0.00121)$ & $(0.000555)$ & $(0.000610)$ & $(0.00318)$ & $(0.00448)$ & $(0.000826)$ & $(0.000828)$ \\
\hline \multirow[t]{2}{*}{ MOODY } & -0.863 & -1.109 & -2.737 & -3.355 & 1.090 & -1.295 & 0.0502 & -3.342 & -4.777 & -3.506 & -3.419 \\
\hline & $(3.165)$ & $(3.115)$ & $(1.810)$ & (3.101) & $(2.783)$ & (3.779) & $(2.403)$ & $(2.563)$ & $(4.103)$ & (3.036) & $(3.047)$ \\
\hline \multirow{2}{*}{\multicolumn{2}{|c|}{ DEFICIT }} & 1.391 & 0.585 & 1.655 & 1.710 & 2.017 & 1.995 & -1.924 & 0.800 & 1.204 & 1.329 \\
\hline & & $(2.230)$ & $(1.580)$ & $(2.257)$ & $(1.752)$ & $(1.790)$ & $(1.661)$ & $(2.148)$ & $(3.215)$ & $(2.230)$ & $(2.251)$ \\
\hline \multicolumn{2}{|l|}{ BAILOUT } & $\begin{array}{l}-3.559 \\
(3.079)\end{array}$ & $\begin{array}{l}-2.480 \\
(2.117)\end{array}$ & $\begin{array}{l}-3.745 \\
(3114)\end{array}$ & $\begin{array}{l}-0.995 \\
(2.330)\end{array}$ & $\begin{array}{l}0.402 \\
(2.383)\end{array}$ & $\begin{array}{l}0.0205 \\
(2.210)\end{array}$ & $\begin{array}{l}-6.579^{* *} \\
(3.036)\end{array}$ & $\begin{array}{l}-7.376 \\
(4.607)\end{array}$ & $\begin{array}{l}-3.962 \\
(3.090)\end{array}$ & $\begin{array}{l}-3.592 \\
(3.110)\end{array}$ \\
\hline \multirow{2}{*}{\multicolumn{2}{|c|}{ WIT }} & $-38.37^{* * *}$ & $-26.79 * * *$ & $-37.73^{* * *}$ & $-49.74^{* * *}$ & $-36.95 * * *$ & $-44.71^{* * *}$ & 4.344 & $-31.78^{*}$ & $-37.37^{* * *}$ & $-37.57 * * *$ \\
\hline & & $(10.38)$ & $(6.632)$ & $(10.47)$ & $(7.373)$ & $(7.507)$ & $(6.976)$ & $\begin{array}{l}4.044 \\
(10.97)\end{array}$ & $(17.38)$ & $(10.39)$ & $(10.46)$ \\
\hline \multirow{2}{*}{\multicolumn{2}{|c|}{ WIT period }} & $-1.876^{* * * *}$ & $-1.418^{* * *}$ & $-1.782^{* * *}$ & $-1.056^{* *}$ & $-0.834^{*}$ & $-0.947^{* *}$ & $-1.664^{* *}$ & $-2.508^{* *}$ & $-1.868^{* * *}$ & $-1.804^{* * * *}$ \\
\hline & & $(0.639)$ & $(0.458)$ & $(0.667)$ & $(0.473)$ & $(0.501)$ & $(0.448)$ & $(0.657)$ & $(1.009)$ & $(0.664)$ & $(0.666)$ \\
\hline APP & & -9.463 & 5.348 & -8.582 & 4.352 & 7.325 & 5.484 & 4.833 & -17.58 & -8.388 & -8.562 \\
\hline & & $(9.356)$ & $(6.623)$ & $(9.465)$ & $(7.357)$ & $(7.516)$ & $(6.979)$ & $(8.842)$ & $(13.21)$ & $(9.383)$ & $(9.461)$ \\
\hline APP period & & $-1.227^{* *}$ & $-0.849^{* *}$ & $-1.175^{* *}$ & $-0.771^{*}$ & -0.592 & -0.662 & $-1.234^{* *}$ & $-1.743^{*}$ & $-1.228^{* *}$ & $-1.207^{* *}$ \\
\hline & & $(0.568)$ & $(0.411)$ & $(0.592)$ & $(0.446)$ & $(0.461)$ & $(0.416)$ & $(0.614)$ & $(0.898)$ & $(0.590)$ & $(0.589)$ \\
\hline Two pack & & -12.17 & -4.199 & $-17.73^{*}$ & -7.455 & 8.803 & 0.900 & -8.304 & $-45.82^{* * *}$ & $-19.13^{*}$ & $-17.82^{*}$ \\
\hline & & $(10.37)$ & $(6.632)$ & $(10.46)$ & $(7.340)$ & $(7.492)$ & $(6.960)$ & $(10.96)$ & $(17.39)$ & $(10.39)$ & $(10.46)$ \\
\hline SSM & & -16.72 & $-21.82^{* * *}$ & -16.01 & $-37.19^{* * *}$ & $-19.72^{* * *}$ & $-29.64^{* * *}$ & -8.295 & -0.139 & -15.73 & -15.81 \\
\hline & & (10.38) & $(6.646)$ & (10.49) & $(7.367)$ & $(7.533)$ & $(6.982)$ & (10.98) & $(17.45)$ & $(10.42)$ & (10.48) \\
\hline BICC & & -8.279 & -8.149 & -9.366 & -8.314 & $-16.09^{* *}$ & -11.21 & -5.830 & -7.833 & -9.402 & -9.304 \\
\hline & & $(9.308)$ & (6.590) & (9.417) & $(7.327)$ & $(7.522)$ & $(6.95$ & $(8.79$ & (13.14) & (9.335) & (9.412) \\
\hline SRM & & 9.816 & 2.595 & 9.813 & 9.400 & 6.48 & 8.0 & -0.9 & 10. & & 9.788 \\
\hline & & $(9.311)$ & (6.591) & (9.418) & $(7.328)$ & $(7.479)$ & (6.949) & (8.795) & (13.14) & (9.336) & (9.413) \\
\hline EDIS & & $\begin{array}{l}-0.500 \\
(9.308)\end{array}$ & $\begin{array}{l}-4.690 \\
(6.590)\end{array}$ & $\begin{array}{l}-0.378 \\
(9.416\end{array}$ & $\begin{array}{l}-5.130 \\
(7.320\end{array}$ & $\begin{array}{l}-8.181 \\
(7.477\end{array}$ & $\begin{array}{l}-6.658 \\
6.945)\end{array}$ & $\begin{array}{l}-3.381 \\
(8.790\end{array}$ & $\begin{array}{l}4.454 \\
(1314)\end{array}$ & $\begin{array}{l}-0.333 \\
(9.334)\end{array}$ & $\begin{array}{l}-0.496 \\
(9.411\end{array}$ \\
\hline EMF & & $\begin{array}{l}(9.308) \\
2.007\end{array}$ & $\begin{array}{l}(6.590) \\
0.490\end{array}$ & $(9.416)$ & $(7.320)$ & $\begin{array}{l}(7.477) \\
-0.598\end{array}$ & $(6.945)$ & $\begin{array}{l}(8.790) \\
0.482\end{array}$ & $\begin{array}{l}(13.14) \\
2.433\end{array}$ & $(9.334)$ & $(9.411)$ \\
\hline EIMT & & $(9.450)$ & $\begin{array}{l}0.490 \\
(6.688)\end{array}$ & $\begin{array}{l}1.311 \\
(9.562)\end{array}$ & $\begin{array}{l}1.901 \\
(7.435)\end{array}$ & $\begin{array}{l}-0.598 \\
(7.595)\end{array}$ & $\begin{array}{l}0.576 \\
(7.054)\end{array}$ & $\begin{array}{l}0.482 \\
(8.933)\end{array}$ & $\begin{array}{l}2.433 \\
(13.35)\end{array}$ & $\begin{array}{l}1.795 \\
(9.506)\end{array}$ & $\begin{array}{l}0.171 \\
(9.518)\end{array}$ \\
\hline PR5 & & $-35.93^{* * *}$ & $-11.43^{*}$ & $-36.20^{* * *}$ & -5.901 & -6.096 & -7.496 & -14.06 & $-52.61^{* * * *}$ & $-35.95 * * *$ & $-35.92^{* * *}$ \\
\hline & & $(9.319)$ & $(6.605)$ & $(9.438)$ & $(7.349)$ & $(7.522)$ & $(6.970)$ & $(8.792)$ & $(13.18)$ & $(9.359)$ & $(9.433)$ \\
\hline Constant & $-2.747 * * *$ & $-134.9 * * *$ & $-65.47 * * *$ & $-129.5^{* * *}$ & 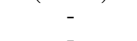 & & $-28.98^{* * * *}$ & $-95.76 * * *$ & $-173.2^{* * * *}$ & -1.596 & -1.606 \\
\hline & & & & YE & $\mathrm{YF}$ & $\mathrm{YE}$ & & & & & \\
\hline $\begin{array}{l}\text { Observations } \\
\text { Obs }\end{array}$ & 12,407 & 12,407 & 9,864 & $\begin{array}{c}\mathrm{Y}, 2 \mathrm{~S} \\
12,200\end{array}$ & $\begin{array}{l}\text { YES } \\
2,521\end{array}$ & $\begin{array}{l}\text { Y ES } \\
2,520\end{array}$ & $\begin{array}{l}\text { YES } \\
5,041\end{array}$ & $\begin{array}{r}\text { YES } \\
4,661\end{array}$ & $\begin{array}{l}\text { YES } \\
7,159\end{array}$ & $\begin{array}{c}\text { YES } \\
12,175\end{array}$ & $\begin{array}{c}\text { YES } \\
12,195\end{array}$ \\
\hline Countries & 5 & 5 & 4 & 5 & 1 & 1 & 2 & 2 & 3 & 5 & 5 \\
\hline R-squared & 0.018 & 0.028 & 0.093 & 0.033 & 0.222 & 0.222 & 0.202 & 0.131 & 0.048 & 0.025 & 0.023 \\
\hline
\end{tabular}

${ }^{* *},{ }^{*}$ : significance at the 1 percent, 5 percent and 10 percent levels. Robust standard errors in parenthesis.

Notes: (1) Limited subset of control variables; (2) All controls; (3) No Greece; (4) Introduction of 1 lag of the dependent variable; (5) Only Spain; (6) Only Italy; (7) Spain and Italy; (8) Ireland and Portugal; (9) Ireland, Portugal and Greece; (10) Events are only the financial-banking subset; (11)

$\mathrm{CMU}$ decisions are added to the baseline decisions. Columns 1 to 9 include 39 relevant decisions on financial and fiscal integration for the euro area as a whole; column 10 contains 32 decisions (only financial integration) and column 11 includes 54 decisions. 
Table 6: Results using as dependent variable the 10-year spread with respect to Germany.

\begin{tabular}{|c|c|c|c|c|c|c|c|c|c|c|c|}
\hline VARIABLES & (1) & (2) & (3) & (4) & (5) & (6) & (7) & (8) & (9) & (10) & (11) \\
\hline Decision t-3 & $2.978^{*}$ & 2.647 & $3.092^{* * *}$ & $2.817^{*}$ & $4.012 * * *$ & $4.282 * * *$ & $4.115^{* * *}$ & 1.717 & 1.243 & $3.928^{* *}$ & 2.199 \\
\hline & (1.694) & $(1.630)$ & $(1.127)$ & (1.658) & $(1.305)$ & $(1.339)$ & $(1.245)$ & $(1.545)$ & $(2.352)$ & $(1.794)$ & $(1.426)$ \\
\hline Decision $t-2$ & $\begin{array}{l}2.330 \\
(1.704)\end{array}$ & $\begin{array}{c}2.249 \\
(1.641)\end{array}$ & $\begin{array}{c}0.329 \\
(1.132)\end{array}$ & $\begin{array}{c}2.688 \\
(1.665)\end{array}$ & $\begin{array}{l}0.820 \\
(1.310)\end{array}$ & $\begin{array}{l}-0.476 \\
(.346)\end{array}$ & $\begin{array}{c}0.294 \\
(1.249)\end{array}$ & $\begin{array}{l}0.0221 \\
(1.548)\end{array}$ & $\begin{array}{l}4.497^{*} \\
(2.365)\end{array}$ & $\begin{array}{l}3.643^{* *} \\
(1.802)\end{array}$ & $\begin{array}{c}2.284 \\
(1.428)\end{array}$ \\
\hline Decision $\mathrm{t}-1$ & -1.348 & $\begin{array}{l}(1.041) \\
-0.979\end{array}$ & -0.282 & $\begin{array}{l}(1.003) \\
-0.821\end{array}$ & -0.787 & 0.371 & 0.00185 & $\begin{array}{l}-1.635 \\
-1.061\end{array}$ & $\begin{array}{l}-2.304 \\
-2.304\end{array}$ & $\begin{array}{l}-1.099 \\
-1.00\end{array}$ & $\begin{array}{l}(1.428) \\
-0.501\end{array}$ \\
\hline & $\begin{array}{l}(1.681) \\
\text { (1) }\end{array}$ & $(1.643)$ & $(1.133)$ & $(1.697)$ & $(1.332)$ & (1.363) & $(1.268)$ & $(1.573)$ & $(2.405)$ & $(1.833)$ & $(1.450)$ \\
\hline Decision $\mathrm{t}$ & $\begin{array}{l}-0.545 \\
(1.680)\end{array}$ & $\begin{array}{l}3.483^{* * *} \\
(1.639)\end{array}$ & $\begin{array}{c}4.158^{* * * *} \\
(1.130)\end{array}$ & $\begin{array}{l}3.308^{* * *} \\
(1.669)\end{array}$ & $\begin{array}{l}3.315^{* * *} \\
(1.306)\end{array}$ & $\begin{array}{l}3.164^{* * *} \\
(1.345)\end{array}$ & $\begin{array}{c}3.279^{* * * *} \\
(1.246)\end{array}$ & $\begin{array}{c}4.768^{* * * *} \\
(1.546)\end{array}$ & $\begin{array}{l}2.370 \\
(2.366)\end{array}$ & $\begin{array}{l}3.327^{*} \\
(1.798)\end{array}$ & $\begin{array}{l}3.024^{* * *} \\
(1.430)\end{array}$ \\
\hline Decision $t+1$ & $-4.440^{* * *}$ & $-4.695^{* * *}$ & $-3.414^{* * *}$ & $-4.695^{* * *}$ & $-2.662^{* *}$ & $\begin{array}{l}-1.732 \\
\end{array}$ & $-2.175^{*}$ & $-5.604^{* * *}$ & $-6.583^{* * *}$ & $-3.800^{* *}$ & $-3.118^{* *}$ \\
\hline e & $(1.679)$ & $(1.627)$ & $(1.122)$ & $(1.657)$ & $(1.296)$ & $(1.331)$ & $(1.237)$ & $(1.534)$ & $(2.349)$ & $(1.806)$ & $(1.421)$ \\
\hline Decision $t+2$ & 0.142 & -0.346 & -0.887 & -0.820 & -1.254 & -0.953 & -1.213 & 0.849 & -0.334 & 0.382 & -1.191 \\
\hline 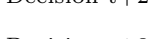 & $(1.679)$ & $(1.616)$ & (1.115) & $(1.646)$ & $\begin{array}{l}(1.289) \\
\text { (1.289 }\end{array}$ & (1.323) & $\begin{array}{l}(1.229) \\
\text { (1.22) }\end{array}$ & $(1.524)$ & $\begin{array}{l}(2.332) \\
\text { (2. }\end{array}$ & $(1.801)$ & $\begin{array}{l}(1.417) \\
\text { (1) }\end{array}$ \\
\hline Decision $t+3$ & $\begin{array}{l}-0.629 \\
(1.695)\end{array}$ & $\begin{array}{l}-0.470 \\
(1.629)\end{array}$ & $\begin{array}{l}-0.315 \\
(1.121)\end{array}$ & $\begin{array}{l}-0.550 \\
(1.658)\end{array}$ & $\begin{array}{c}0.331 \\
(1.289)\end{array}$ & $\begin{array}{l}-0.519 \\
(1.346)\end{array}$ & $\begin{array}{c}-0.00566 \\
(1.234)\end{array}$ & $\begin{array}{l}-0.405 \\
(1.546)\end{array}$ & $\begin{array}{l}-0.820 \\
(2.359)\end{array}$ & $\begin{array}{l}-0.822 \\
(1.818)\end{array}$ & $\begin{array}{l}-0.970 \\
(1.425)\end{array}$ \\
\hline BIDASK & $-2.753^{* * *}$ & $-3.016^{* * *}$ & 0.672 & $-3.058^{* * *}$ & 3.679 & $\begin{array}{l}(1.040) \\
-4.216\end{array}$ & 0.376 & $\begin{array}{l}(1.040) \\
-0.571\end{array}$ & $-3.599 * * *$ & $-3.162^{* * * *}$ & $-3.079 * * *$ \\
\hline & $(0.676)$ & $(0.670)$ & (1.034) & $(0.691)$ & $(8.823)$ & (15.68) & $(9.265)$ & $(1.104)$ & $(0.704)$ & $(0.677)$ & $(0.677)$ \\
\hline$\triangle$ EONIA & 0.284 & 0.468 & 2.082 & -0.196 & $\begin{array}{l}-0.786 \\
-6250\end{array}$ & -1.782 & -1.356 & $5.067^{*}$ & -0.839 & 0.968 & $\begin{array}{l}-0.213 \\
-(2000\end{array}$ \\
\hline & $(2.997)$ & $\begin{array}{l}(2.895) \\
\text { (2. }\end{array}$ & (1.993) & $(2.933)$ & $(2.359)$ & $(2.425)$ & $(2.250)$ & $(2.753)$ & $(4.123)$ & $(2.946)$ & $(2.929)$ \\
\hline VIX & $0.217^{* * *}$ & $0.183^{* * *}$ & 0.0122 & $0.199^{* * * *}$ & -0.0376 & -0.0346 & $\begin{array}{l}-0.0269 \\
-(0.02303)\end{array}$ & $\begin{array}{l}-0.0125 \\
0.0392\end{array}$ & $0.310^{* * * *}$ & $0.206^{* * * *}$ & $0.196^{* * * *}$ \\
\hline & $(0.0360)$ & $(0.0425)$ & $(0.0300)$ & $(0.0445)$ & $(0.0321)$ & $(0.0321)$ & $(0.0303)$ & $(0.0392)$ & $(0.0643)$ & $(0.0442)$ & $(0.0442)$ \\
\hline ECB & $\begin{array}{c}1.137 \\
(1.009)\end{array}$ & $\begin{array}{l}-0.0451 \\
(1.050)\end{array}$ & $\begin{array}{c}-1.343^{*} \\
(0.718)\end{array}$ & $\begin{array}{r}-0.0959 \\
(1.069)\end{array}$ & $\begin{array}{c}-1.675^{* *} \\
(0.838)\end{array}$ & $\begin{array}{c}-2.419 * * * \\
(0.858)\end{array}$ & $\begin{array}{c}-2.101 * * * \\
(0.799)\end{array}$ & $\begin{array}{l}-0.432 \\
(1.001)\end{array}$ & $\begin{array}{l}1.630 \\
(1519)\end{array}$ & $\begin{array}{l}-0.114 \\
(1.064\end{array}$ & $\begin{array}{l}-0.108 \\
(1.068\end{array}$ \\
\hline$\triangle \mathrm{STOCK}$ & $-0.0240^{* * *}$ & $-0.0211^{* * *}$ & $-0.0163^{* * *}$ & $-0.0207^{* * * *}$ & $-0.0258^{* * *}$ & $-0.0131^{* * *}$ & $-0.0149 * * *$ & $-0.0662^{* * *}$ & $-0.0743^{* * *}$ & $-0.0215^{* * *}$ & $\begin{array}{c}(1.068) \\
-0.0214^{* * *}\end{array}$ \\
\hline 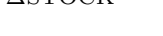 & $(0.000827)$ & $(0.000833)$ & $(0.000559)$ & $(0.000842)$ & $(0.00125)$ & $(0.000572)$ & $(0.000630)$ & $(0.00316)$ & $(0.00450)$ & $(0.000828)$ & $(0.000829)$ \\
\hline MOODY & -0.224 & -0.499 & -1.465 & -2.831 & 2.741 & 0.702 & 1.748 & -2.384 & -4.7 & -2.857 & -2.833 \\
\hline DEFICIT & $(3.175)$ & $(3.120)$ & $(1.789)$ & $(3.108)$ & $(2.866)$ & (3.897) & $(2.478)$ & $(2.549)$ & $(4.111)$ & (3.047) & $(3.056)$ \\
\hline DEFICIT & & $\begin{array}{l}0.653 \\
(2.238)\end{array}$ & $\begin{array}{l}-0.219 \\
(1.552)\end{array}$ & $\begin{array}{l}0.967 \\
(2.275)\end{array}$ & $\begin{array}{l}1.205 \\
(1.803)\end{array}$ & $\begin{array}{l}1.524 \\
(1846\end{array}$ & 1.499 & -2.780 & -0.0107 & 0.549 & 0.412 \\
\hline BAILOUT & & $\begin{array}{l}2.230) \\
-1.662\end{array}$ & $\begin{array}{l}(1.552) \\
-0.444\end{array}$ & $\begin{array}{l}(2.275) \\
-1.791\end{array}$ & (1.803) & $(1.846)$ & $(1.718)$ & $(2.131)$ & $(3.228)$ & $(2.253)$ & $(2.267)$ \\
\hline & & $(3.094)$ & $\begin{array}{l}-0.444 \\
(2.082)\end{array}$ & $\begin{array}{l}-1.791 \\
(3.141)\end{array}$ & $\begin{array}{l}0.728 \\
(2.398)\end{array}$ & $\begin{array}{l}2.047 \\
(2.458)\end{array}$ & $\begin{array}{l}1.731 \\
(2.286)\end{array}$ & $\begin{array}{l}-4.617 \\
(3.014)\end{array}$ & $\begin{array}{l}-5.379 \\
(4.628)\end{array}$ & $\begin{array}{l}-2.046 \\
(3.124)\end{array}$ & $\begin{array}{l}-1.750 \\
(3.135\end{array}$ \\
\hline WIT & & $-35.98^{* * *}$ & $-24.53^{* * *}$ & $-35.25^{* * *}$ & $-47.00^{* * *}$ & $-34.33 * * *$ & $\begin{array}{r}(2.286) \\
-42.02^{* * *}\end{array}$ & 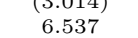 & $\begin{array}{l}(4.628) \\
-29.34^{*}\end{array}$ & $\begin{array}{r}(3.124) \\
-34.99^{* * *}\end{array}$ & $\begin{array}{l}(3.135) \\
-35.15^{* * *}\end{array}$ \\
\hline & & $(10.44)$ & $(6.533)$ & (10.57) & $(7.590)$ & $(7.745)$ & $(7.219)$ & $(10.92)$ & $(17.46)$ & $(10.51)$ & $\begin{array}{r}-30.15 \\
(10.56)\end{array}$ \\
\hline WIT period & & $-1.932 * * *$ & $-1.453^{* * * *}$ & $-1.929 * * *$ & $-1.170^{* *}$ & $-1.037^{* *}$ & $-1.092^{* *}$ & $-1.748^{* * * *}$ & $-2.661^{* * * *}$ & $-2.002^{* * * *}$ & $-1.937^{* * * *}$ \\
\hline & & $(0.650)$ & $(0.459)$ & $(0.682)$ & $(0.491)$ & $(0.525)$ & $(0.469)$ & $(0.656)$ & $(1.024)$ & $(0.678)$ & $(0.678)$ \\
\hline APP & & $-18.29^{*}$ & -3.946 & $-17.78^{*}$ & -3.277 & 0.234 & -1.980 & $\begin{array}{l}-3.452 \\
\end{array}$ & $-27.13^{* *}$ & $-17.67^{*}$ & $-17.73^{*}$ \\
\hline & & $(9.392)$ & $(6.503)$ & $(9.537)$ & $(7.576)$ & $(7.756)$ & $(7.223)$ & $(8.772)$ & $(13.26)$ & $(9.477)$ & $(9.528)$ \\
\hline APP period & & $-1.283^{* *}$ & $-0.860^{* *}$ & $-1.250^{* *}$ & $-0.842^{*}$ & -0.761 & $-0.771^{*}$ & $-1.274^{* *}$ & $-1.823^{* *}$ & $-1.294^{* *}$ & $-1.280^{* * *}$ \\
\hline & & $(0.577)$ & $(0.412)$ & $(0.605)$ & $(0.464)$ & $(0.482)$ & $(0.435)$ & $(0.613)$ & $(0.911)$ & $(0.601)$ & $(0.600)$ \\
\hline Two pack & & $-18.61^{*}$ & $\begin{array}{l}-7.322 \\
6530\end{array}$ & $-23.87^{* *}$ & $\begin{array}{l}-11.86 \\
(7557)\end{array}$ & $\begin{array}{l}3.552 \\
(7730\end{array}$ & $\begin{array}{l}-3.771 \\
-7\end{array}$ & $\begin{array}{l}-12.72 \\
(10.91\end{array}$ & $-52.82^{* * *}$ & $-23.94^{* *}$ & $-23.10^{* *}$ \\
\hline & & $(10.44)$ & $(6.532)$ & $(10.56)$ & $(7.557)$ & $(7.730)$ & $(7.202)$ & (10.91) & (17.47) & (10.51) & $(10.55)$ \\
\hline SSM & & $-20.86^{* *}$ & $-26.69^{* * *}$ & $-20.17^{*}$ & $-40.95^{* * *}$ & $-23.25^{* * * *}$ & $-33.30^{* * * *}$ & $\begin{array}{l}-12.24 \\
(10.93\end{array}$ & $\begin{array}{l}-4.161 \\
(1755)\end{array}$ & $\begin{array}{l}-19.86^{*} \\
(10.54\end{array}$ & $\begin{array}{l}-19.94^{*} \\
(10.59)\end{array}$ \\
\hline $\mathrm{BICC}$ & & $\begin{array}{l}(10.45) \\
-9.004\end{array}$ & $\begin{array}{l}(6.555) \\
-8.616\end{array}$ & & & $\begin{array}{l}(7.763) \\
-16.71^{* * *}\end{array}$ & $\begin{array}{l}(7.219) \\
-1205^{*}\end{array}$ & (10.93) & $\begin{array}{l}(17.55) \\
-8777\end{array}$ & $(10.54)$ & $(10.59)$ \\
\hline & & $(9.343)$ & $\begin{array}{l}-8.010 \\
(6.469)\end{array}$ & $\begin{array}{l}-1.29 \\
(9.488)\end{array}$ & $\begin{array}{l}-9.368 \\
(7.544)\end{array}$ & $\begin{array}{r}-16.71 * * \\
(7.762)\end{array}$ & $\begin{array}{l}-12.05^{\wedge} \\
(7.200)\end{array}$ & $\begin{array}{l}-6.987 \\
(8.723)\end{array}$ & $\begin{array}{l}-8.747 \\
(13.19)\end{array}$ & $\begin{array}{l}-10.35 \\
(9.428)\end{array}$ & $\begin{array}{l}-10.26 \\
(9.479)\end{array}$ \\
\hline SRM & & $15.66^{*}$ & 8.386 & $15.79^{*}$ & $14.99^{* *}$ & 12.11 & $13.67^{*}$ & 4.918 & 16.86 & $15.85^{*}$ & $15.77^{*}$ \\
\hline & & $(9.345)$ & $(6.470)$ & $(9.489)$ & $(7.545)$ & $(7.717)$ & $(7.191)$ & $(8.725)$ & $(13.19)$ & $(9.429)$ & $(9.479)$ \\
\hline EDIS & & 3.557 & -0.472 & 3.760 & -1.318 & -4.681 & -2.965 & 0.587 & 8.698 & 3.789 & 3.620 \\
\hline S & & (9.343) & $(6.470)$ & $(9.488)$ & $(7.537)$ & $(7.715)$ & $(7.187)$ & $(8.721)$ & $\begin{array}{l}(13.19) \\
\text { (13. }\end{array}$ & $(9.428)$ & $(9.478)$ \\
\hline EMF & & 3.948 & 2.285 & 3.160 & 3.759 & 1.116 & 2.327 & 2.268 & 4.277 & 3.490 & 2.764 \\
\hline PR5 & & $\begin{array}{c}(9.485) \\
-27.94^{* * *}\end{array}$ & $\begin{array}{l}(6.566) \\
-3.374\end{array}$ & $\begin{array}{c}(9.635) \\
-28.08^{* * *}\end{array}$ & $\begin{array}{c}(7.655) \\
1.342\end{array}$ & $\begin{array}{c}(7.836) \\
0.951\end{array}$ & $\begin{array}{l}(7.299) \\
-0.295\end{array}$ & $\begin{array}{l}(8.862) \\
-6.683\end{array}$ & $\begin{array}{c}(13.40) \\
-44.25^{* * *}\end{array}$ & $\begin{array}{c}(9.602) \\
-27.89^{* * *}\end{array}$ & $\begin{aligned} &(9.586) \\
&-27.87 * * *\end{aligned}$ \\
\hline & & $(9.358)$ & $(6.494)$ & $(9.519)$ & (7.563) & $(7.751)$ & $(7.207)$ & $(8.723)$ & $(13.25)$ & $(9.458)$ & $(9.507)$ \\
\hline Constant & $\begin{array}{c}-2.725^{* * *} \\
(0.919)\end{array}$ & $\begin{array}{c}-141.7^{* * * *} \\
(9.657)\end{array}$ & $\begin{array}{c}-73.04^{* * *} \\
(6.690)\end{array}$ & $\begin{array}{c}-135.5^{* * *} \\
(9.832)\end{array}$ & - & - & $\begin{array}{c}-35.33 * * * \\
(7.451)\end{array}$ & $\begin{array}{c}-102.2 * * * \\
(9.003)\end{array}$ & $\begin{array}{c}-178.2^{* * * *} \\
(13.71)\end{array}$ & $\begin{array}{l}-1.564 \\
(1.352)\end{array}$ & $\begin{array}{l}-1.583 \\
(1.352)\end{array}$ \\
\hline LAG & $\mathrm{NC}$ & $\mathrm{NC}$ & & & $\mathrm{YE}$ & $\mathrm{YE}$ & $\mathrm{Yl}$ & $\mathrm{Y} 1$ & $\mathrm{YF}$ & YES & $\mathrm{YE}$ \\
\hline Observations & 12,384 & 12,384 & 9,844 & 12,187 & 2,519 & 2,516 & 5,035 & 4,655 & 7,152 & 12,162 & 12,182 \\
\hline Countries & 5 & 5 & 4 & 5 & 1 & 1 & 2 & 2 & 3 & 5 & 5 \\
\hline R-squared & 0.018 & 0.029 & 0.096 & 0.036 & 0.220 & 0.219 & 0.200 & 0.134 & 0.052 & 0.027 & 0.025 \\
\hline
\end{tabular}

***, ${ }^{* *},{ }^{*}$ : significance at the 1 percent, 5 percent and 10 percent levels. Robust standard errors in parenthesis.

Notes: (1) Limited subset of control variables; (2) All controls; (3) No Greece; (4) Introduction of 1 lag of the dependent variable; (5) Only Spain; (6) Only Italy; (7) Spain and Italy; (8) Ireland and Portugal; (9) Ireland, Portugal and Greece; (10) Events are only the financial-banking subset; (11)

CMU decisions are added to the baseline decisions. Columns 1 to 9 include 39 relevant decisions on financial and fiscal integration for the euro area as a whole; column 10 contains 32 decisions (only financial integration) and column 11 includes 54 decisions. 
Table 7: Results: placebo exercise, when $t=0$ corresponds to the meeting prior to the decision (dependent variable: 10-year spread with respect to OIS).

\begin{tabular}{|c|c|c|c|c|c|c|c|c|c|c|c|}
\hline VARIABLES & (1) & (2) & (3) & (4) & (5) & (6) & (7) & (8) & (9) & (10) & (11) \\
\hline$t-3$ & -1.974 & -1.876 & -1.230 & -1.900 & -1.705 & -0.992 & -1.332 & -1.673 & -2.636 & -1.408 & -0.236 \\
\hline & $\begin{array}{l}(2.010) \\
3.00 *\end{array}$ & $(1.946)$ & (1.344) & $(1.970)$ & (1.488) & $(1.518)$ & $(1.410)$ & $(1.827)$ & $(2.817)$ & $(2.228)$ & (1.503) \\
\hline$t-2$ & $\begin{array}{l}3.802^{*} \\
(2.052)\end{array}$ & $\begin{array}{l}3.796^{*} \\
(1.987\end{array}$ & -0.0994 & $\begin{array}{l}3.665^{*} \\
(2.010)\end{array}$ & $\begin{array}{l}0.241 \\
(1522)\end{array}$ & $\begin{array}{l}0.321 \\
(1558)\end{array}$ & $\begin{array}{c}0.262 \\
(1.444)\end{array}$ & -0.0843 & $6.174^{* *}$ & $\begin{array}{l}0.852 \\
(2.292)\end{array}$ & 2.232 \\
\hline & $\begin{array}{c}(2.052) \\
2.290\end{array}$ & $\begin{array}{l}(1.987) \\
2.319\end{array}$ & $\begin{array}{l}(1.372) \\
1.314\end{array}$ & $\begin{array}{l}(2.010) \\
2.492\end{array}$ & $\begin{array}{c}(1.522) \\
0.869\end{array}$ & $\begin{array}{c}(1.558) \\
0.753\end{array}$ & $\begin{array}{l}(1.444) \\
0.971\end{array}$ & $\begin{array}{l}(1.867) \\
1.569\end{array}$ & $\begin{array}{l}(2.878) \\
3.773)\end{array}$ & $\begin{array}{l}(2.292) \\
6.146 * * *\end{array}$ & $\begin{array}{l}(1.521) \\
-0.177\end{array}$ \\
\hline$t-1$ & $\begin{array}{l}2.290 \\
(2.060)\end{array}$ & $\begin{array}{l}2.319 \\
(1.993)\end{array}$ & $\begin{array}{l}1.314 \\
(1.376)\end{array}$ & $\begin{array}{c}2.492 \\
(2.017)\end{array}$ & $\begin{array}{l}\text { (1.869 } \\
(1.533)\end{array}$ & $\begin{array}{l}0.753 \\
(1.566)\end{array}$ & $\begin{array}{l}0.971 \\
(1.451)\end{array}$ & $\begin{array}{l}1.569 \\
(1.872)\end{array}$ & $\begin{array}{l}3.886) \\
(2.873\end{array}$ & 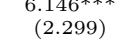 & $\begin{array}{l}-0.177 \\
(1.527)\end{array}$ \\
\hline $\mathrm{t}$ & $-5.762 * * *$ & -0.361 & 0.124 & -0.380 & -1.087 & 0.213 & -0.309 & 0.608 & -1.284 & 0.733 & $2.748^{*}$ \\
\hline & $(2.007)$ & $(2.028)$ & $(1.400)$ & (2.099) & (1.590) & (1.628) & (1.509) & (1.953) & (3.008) & $(2.355)$ & (1.538) \\
\hline$t+1$ & $-6.141^{* * * *}$ & $\begin{array}{l}-0.995 \\
01085\end{array}$ & -0.538 & -1.394 & 1.663 & 0.933 & 1.338 & -2.163 & $\begin{array}{l}-3.953 \\
\end{array}$ & -0.183 & -1.148 \\
\hline & $\begin{array}{l}(2.010) \\
-2.284\end{array}$ & $\begin{array}{l}(1.985) \\
-2484\end{array}$ & $\begin{array}{l}(1.370) \\
-1.689\end{array}$ & $\begin{array}{l}(2.008) \\
-2819\end{array}$ & $(1.523)$ & $\begin{array}{l}(1.558) \\
-1296\end{array}$ & $(1.444)$ & $(1.867)$ & $(2.875)$ & $(2.288)$ & $(1.520)$ \\
\hline$t+2$ & $\begin{array}{l}-2.284 \\
(2.020)\end{array}$ & $\begin{array}{l}-2.484 \\
(1.955)\end{array}$ & $\begin{array}{l}-1.689 \\
(1.351)\end{array}$ & $\begin{array}{l}-2.819 \\
(1.979)\end{array}$ & $\begin{array}{l}-0.861 \\
(1.505)\end{array}$ & $\begin{array}{l}-1.296 \\
(1.535)\end{array}$ & -1.138 & $\begin{array}{l}-1.203 \\
(1.839)\end{array}$ & $\begin{array}{l}-4.109 \\
(2.32)\end{array}$ & $\begin{array}{l}-1.970 \\
(2.237\end{array}$ & $\begin{array}{l}-1.391 \\
(1508)\end{array}$ \\
\hline & $\begin{array}{l}(2.020) \\
-1.647\end{array}$ & $\begin{array}{l}(1.955) \\
-1.572\end{array}$ & $\begin{array}{l}(1.351) \\
-1.076\end{array}$ & $\begin{array}{l}(1.979) \\
-1.723\end{array}$ & $\begin{array}{l}(1.505) \\
-0.694\end{array}$ & $\begin{array}{l}(1.535) \\
-0.283\end{array}$ & $\begin{array}{l}(1.424) \\
-0.590\end{array}$ & $\begin{array}{l}(1.839) \\
-0.525\end{array}$ & $\begin{array}{l}(2.832) \\
-1.970\end{array}$ & $\begin{array}{l}(2.237) \\
-1.345\end{array}$ & $\begin{array}{r}(1.508) \\
-0.0885\end{array}$ \\
\hline tas & $\begin{array}{l}-1.047 \\
(2.015)\end{array}$ & $(1.952)$ & $(1.349)$ & $(1.976)$ & $\begin{array}{l}-0.094 \\
(1.492)\end{array}$ & $\begin{array}{l}-0.280 \\
(1.522)\end{array}$ & (1.414) & $\begin{array}{l}-0.020 \\
(1.833)\end{array}$ & $\begin{array}{l}-1.820 \\
(2.827)\end{array}$ & $\begin{array}{l}-1.040 \\
(2.231)\end{array}$ & $\begin{array}{l}-0.0800 \\
(1.500)\end{array}$ \\
\hline BIDASK & $-2.655^{* * * *}$ & $-2.781^{* * * *}$ & 0.734 & -2.776 **** & 3.692 & 0.389 & 1.612 & -0.726 & $-3.328 * * *$ & $-2.775 * * *$ & $-2.783^{* * * *}$ \\
\hline & $(0.693)$ & $(0.686)$ & (1.027) & $(0.701)$ & $(8.492)$ & (14.76) & (8.833) & (1.110) & $(0.719)$ & $(0.686)$ & $(0.686)$ \\
\hline$\triangle$ EONIA & $\begin{array}{l}-0.443 \\
(3.055)\end{array}$ & $\begin{array}{l}-0.383 \\
(2.975)\end{array}$ & $\begin{array}{l}0.607 \\
(2.060)\end{array}$ & $\begin{array}{l}-0.627 \\
(3.005)\end{array}$ & $\begin{array}{l}-1.554 \\
(2.324)\end{array}$ & $\begin{array}{l}-2.560 \\
(2.376)\end{array}$ & $\begin{array}{l}-2.169 \\
(2.202)\end{array}$ & $\begin{array}{l}3.675 \\
(2809)\end{array}$ & $\begin{array}{l}-0.655 \\
(4.266)\end{array}$ & $\begin{array}{l}-0.541 \\
(2.973)\end{array}$ & $\begin{array}{r}-0.649 \\
(2.961)\end{array}$ \\
\hline VIX & $0.227 * * *$ & $0.189^{* * *}$ & $\begin{array}{l}(2.060) \\
0.0150\end{array}$ & $0.200^{* * *}$ & $\begin{array}{l}(2.324) \\
-0.0374\end{array}$ & $\begin{array}{l}(2.376) \\
-0.0361\end{array}$ & $\begin{array}{l}(2.202) \\
-0.0279\end{array}$ & $\begin{array}{l}(2.809) \\
-0.0116\end{array}$ & $\begin{array}{l}(4.266) \\
0.321^{* * *}\end{array}$ & $0.184^{* * *}$ & $\left.0.1867^{* * *}\right)$ \\
\hline (1) & $(0.0366)$ & $(0.0431)$ & $(0.0304)$ & $(0.0444)$ & $(0.0311)$ & $(0.0310)$ & $\begin{array}{l}-0.0279 \\
(0.0292)\end{array}$ & $\begin{array}{l}-0.0116 \\
(0.0398)\end{array}$ & $\begin{array}{l}.321 \\
(0.0651)\end{array}$ & $\begin{array}{l}(0.0430) \\
(0.043\end{array}$ & $(0.0429)$ \\
\hline $\mathrm{ECB}$ & 1.174 & 0.162 & -1.129 & 0.0867 & $-1.356^{*}$ & $-2.171^{* * * *}$ & $-1.826^{* *}$ & -0.215 & 1.727 & 0.0564 & 0.255 \\
\hline & $(1.026)$ & $(1.076)$ & $(0.739))$ & $(1.092)$ & $(0.822)$ & $(0.837)$ & $(0.779)$ & $(1.020)$ & $(1.568)$ & $(1.076)$ & $(1.074)$ \\
\hline$\triangle \mathrm{STOCK}$ & $-0.0235 * * *$ & $-0.0209^{* * *}$ & $-0.0165^{* * *}$ & $-0.0207^{* * *}$ & $-0.0260^{* * *}$ & $-0.0131^{* * *}$ & $-0.0150^{* * *}$ & $-0.0664 * * *$ & $-0.0720^{* * *}$ & $-0.0208^{* * *}$ & $\begin{array}{c}-0.0209^{* * *} \\
(0.00848\end{array}$ \\
\hline & $(0.000841)$ & $(0.000849)$ & $(0.000571)$ & $(0.000857)$ & $(0.00122)$ & $(0.000553)$ & $(0.000609)$ & $(0.00318)$ & $(0.00464)$ & $(0.000850)$ & $(0.000848)$ \\
\hline MOODY & $\begin{array}{l}-1.592 \\
(3.271)\end{array}$ & $\begin{array}{l}-1.593 \\
(3.226)\end{array}$ & $\begin{array}{l}-2.921 \\
(1.819)\end{array}$ & $\begin{array}{l}-1.498 \\
(3.249)\end{array}$ & $\begin{array}{c}0.612 \\
(2.815)\end{array}$ & $\begin{array}{l}-1.189 \\
(3.765)\end{array}$ & $\begin{array}{l}-0.135 \\
(2.416)\end{array}$ & $\begin{array}{l}-3.644 \\
(2.580)\end{array}$ & $\begin{array}{l}-1.963 \\
(4.287)\end{array}$ & $\begin{array}{l}-1.562 \\
(3.228)\end{array}$ & $\begin{array}{l}-1.503 \\
(3.224)\end{array}$ \\
\hline WIT & & $-38.33^{* * * *}$ & $-26.76^{* * * *}$ & $-37.93^{* * * *}$ & $-49.76 * * *$ & $-36.94^{* * * *}$ & $-44.74^{* * * *}$ & 4.763 & $-32.09^{*}$ & $-38.36^{* * * *}$ & $-38.34^{* * * *}$ \\
\hline & & $(10.68)$ & $(6.681)$ & $(10.78)$ & $(7.421)$ & $(7.527)$ & $(7.010)$ & $(11.07)$ & $(18.08)$ & (10.69) & $(10.67)$ \\
\hline WIT period & & $-1.739 * * *$ & $-1.360^{* * * *}$ & $-1.617^{* *}$ & $-1.110^{* *}$ & $-0.885^{*}$ & $-1.004^{* *}$ & $-1.691^{* *}$ & $-2.148 * *$ & $-1.791^{* * *}$ & $-1.773^{* * *}$ \\
\hline & & $(0.656)$ & $(0.461)$ & $(0.676)$ & $(0.473)$ & $(0.500)$ & $(0.448)$ & $(0.659)$ & $(1.029)$ & $(0.657)$ & $(0.656)$ \\
\hline APP & & $\begin{array}{l}-9.358 \\
(9.586)\end{array}$ & $\begin{array}{l}5.362 \\
(6.667)\end{array}$ & $\begin{array}{l}-8.799 \\
(9696)\end{array}$ & 4.138 & $\begin{array}{l}7.186 \\
(7536)\end{array}$ & $\begin{array}{l}5.297 \\
(7012)\end{array}$ & $\begin{array}{l}4.847 \\
(8.904\end{array}$ & $\begin{array}{l}-17.70 \\
(1363\end{array}$ & $\begin{array}{l}-9.271 \\
\end{array}$ & $\begin{array}{l}-9.457 \\
-0.577\end{array}$ \\
\hline APP period & & $\begin{array}{l}(9.586) \\
-1.040^{*}\end{array}$ & $\begin{array}{l}(6.667) \\
-0.784^{*}\end{array}$ & $\begin{array}{l}(9.696) \\
-0.979\end{array}$ & $\begin{array}{l}(7.404) \\
-0.834 *\end{array}$ & $\begin{array}{l}(7.536) \\
-0.650\end{array}$ & $\begin{array}{l}(7.012) \\
-0.729^{*}\end{array}$ & $\begin{array}{l}(8.904) \\
-1.244 * *\end{array}$ & $\begin{array}{l}(13.63) \\
-1.306\end{array}$ & $\begin{array}{l}(9.597) \\
-1068^{*}\end{array}$ & $\begin{array}{l}(9.577) \\
-1.062^{*}\end{array}$ \\
\hline APP period & & $\begin{array}{l}-1.040^{*} \\
(0.579)\end{array}$ & $\begin{array}{l}-0.184^{*} \\
(0.412)\end{array}$ & $\begin{array}{l}-0.979 \\
(0.597)\end{array}$ & $\begin{array}{l}-0.834 * \\
(0.444)\end{array}$ & $\begin{array}{l}-0.650 \\
(0.457)\end{array}$ & $\begin{array}{l}-0.729 * \\
(0.413)\end{array}$ & $\begin{array}{c}-1.244^{* 2 *} \\
(0.614)\end{array}$ & $\begin{array}{l}-1.306 \\
(0.911)\end{array}$ & $\begin{array}{l}-1.068 \\
(0.580)\end{array}$ & $\begin{array}{l}-1.062^{*} \\
(0.581)\end{array}$ \\
\hline Two pack & & $\begin{array}{l}-12.29 \\
-129\end{array}$ & -4.235 & $\begin{array}{l}0.5913 \\
-15.13\end{array}$ & $\begin{array}{l}\text { (.4.44) } \\
-7.620\end{array}$ & 8.738 & 0.798 & $\begin{array}{l}(.014) \\
-8.150\end{array}$ & $-40.43^{* *}$ & $\begin{array}{l}(1.080) \\
-12.15\end{array}$ & $\begin{array}{l}(0.581) \\
-12.32\end{array}$ \\
\hline Wo pack & & (10.67) & $(6.681)$ & (10.78) & $(7.388)$ & $(7.513)$ & $(6.994)$ & (11.06) & $\begin{array}{l}(18.09) \\
(18.09\end{array}$ & $(10.69)$ & $(10.66)$ \\
\hline SSM & & -16.64 & $-21.72^{* * * *}$ & -16.11 & $-37.05^{* * *}$ & $-19.68^{* * *}$ & $-29.58^{* * * *}$ & -7.707 & -0.261 & -16.66 & -16.61 \\
\hline & & & $\begin{array}{l}(6.694) \\
-8.249\end{array}$ & $\begin{array}{l}(10.80) \\
-9.282\end{array}$ & $\begin{array}{l}(7.417) \\
-8.517\end{array}$ & $\begin{array}{l}(7.557) \\
-16.25 * *\end{array}$ & $\begin{array}{l}(7.019) \\
-1139\end{array}$ & $\begin{array}{l}(11.08) \\
-5.909\end{array}$ & $\begin{array}{l}(18.13) \\
-7.667\end{array}$ & & (10.68) \\
\hline BICC & & $\begin{array}{l}-8.384 \\
(9.537)\end{array}$ & $\begin{array}{l}-8.249 \\
(6.633)\end{array}$ & $\begin{array}{r}-9.282 \\
(9.646)\end{array}$ & $\begin{array}{r}-8.517 \\
(7.374)\end{array}$ & $\begin{array}{l}-16.25^{* *} \\
(7.542)\end{array}$ & $\begin{array}{l}-11.39 \\
(6.991)\end{array}$ & $\begin{array}{l}-5.909 \\
(8.854)\end{array}$ & $\begin{array}{l}-7.667 \\
(13.55)\end{array}$ & $\begin{array}{r}-8.359 \\
(9.548)\end{array}$ & $\begin{array}{r}-8.371 \\
-9\end{array}$ \\
\hline SRM & & $\begin{array}{l}\left(\begin{array}{l}9.537) \\
9.879\end{array}\right.\end{array}$ & $\begin{array}{l}(6.633) \\
2.593\end{array}$ & $\begin{array}{l}(9.646) \\
9.861\end{array}$ & $\begin{array}{c}(7.374) \\
9.469\end{array}$ & $\begin{array}{l}(7.542) \\
6.466\end{array}$ & $\begin{array}{l}(6.991) \\
8.055\end{array}$ & $\begin{array}{l}(8.854) \\
-0.857\end{array}$ & $\begin{array}{l}(13.55) \\
10.91\end{array}$ & 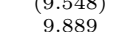 & $(9.528)$ \\
\hline SRMM & & $\begin{array}{l}9.819 \\
(9.540)\end{array}$ & $\begin{array}{l}2.593 \\
(6.634)\end{array}$ & $\begin{array}{l}9.861 \\
(9.647)\end{array}$ & $\begin{array}{r}9.469 \\
(7.375)\end{array}$ & $\begin{array}{l}6.466 \\
(7.499)\end{array}$ & $\begin{array}{l}8.055 \\
(6.982)\end{array}$ & $\begin{array}{l}-0.858 \\
(8.856)\end{array}$ & $\begin{array}{l}10.91 \\
(13.55)\end{array}$ & $\begin{array}{l}9.889 \\
(9.550)\end{array}$ & $\begin{array}{l}9.946 \\
(9.530)\end{array}$ \\
\hline EDIS & & -0.673 & -4.964 & -0.670 & -5.121 & -7.955 & -6.569 & -3.532 & 4.211 & -0.636 & -0.593 \\
\hline & & (9.537) & (6.633) & $(9.645)$ & $(7.367)$ & $(7.496)$ & $(6.978)$ & (8.851) & (13.55) & (9.547) & $(9.528)$ \\
\hline EMF & & 0.360 & -0.605 & $\begin{array}{l}-0.188 \\
(9.644\end{array}$ & 0.956 & -0.450 & 0.335 & -1.525 & -0.488 & 0.325 & 0.414 \\
\hline & & $\begin{array}{l}(9.537) \\
-36.01 * * *\end{array}$ & $\begin{array}{l}(6.633) \\
-11.47^{*}\end{array}$ & $\begin{array}{l}(9.644) \\
-36.28 * *\end{array}$ & $\begin{array}{l}(7.368) \\
-5.849\end{array}$ & $\begin{array}{l}(7.496) \\
-6.129\end{array}$ & $\begin{array}{l}(6.978) \\
-7.521\end{array}$ & $\begin{array}{l}(8.852) \\
-14.05\end{array}$ & (13.55) & $(9.547) *$ & (9.528) \\
\hline PR5 & & $\begin{array}{l}-36.01178 * \\
(9.548)\end{array}$ & $\begin{array}{l}-11.47^{*} \\
(6.649)\end{array}$ & $\begin{array}{l}-36.28 \times 2) \\
(9.662)\end{array}$ & $\begin{array}{l}-5.849 \\
(7.399)\end{array}$ & $\begin{array}{l}-6.129 \\
(7.546)\end{array}$ & $\begin{array}{l}-7.521 \\
(7.006)\end{array}$ & $\begin{array}{l}-14.05 \\
(8.854)\end{array}$ & $\begin{array}{l}-52.72^{* * *} \\
(13.58)\end{array}$ & $\begin{array}{c}-36.044^{* 2 *} \\
(9.559)\end{array}$ & $\begin{array}{l}-35.96 * 96 * \\
(9.540)\end{array}$ \\
\hline Constant & $\begin{array}{c}-2.810^{* * *} \\
(0.935)\end{array}$ & $\begin{array}{c}-131.9^{* * *} \\
(10.09)\end{array}$ & $\begin{array}{c}-62.49 * * * \\
(7.011)\end{array}$ & $\begin{array}{c}-128.0^{* * * *} \\
(10.23)\end{array}$ & $\begin{array}{l}0 \\
(0)\end{array}$ & $\begin{array}{l}0 \\
(0)\end{array}$ & $\begin{array}{c}-28.35 * * * \\
(7.435)\end{array}$ & $\begin{array}{c}-90.69 * * * * \\
(9.385)\end{array}$ & $\begin{array}{c}-171.11^{* * * *} \\
(14.42)\end{array}$ & $\begin{array}{c}-133.7^{* * * *} \\
(10.24)\end{array}$ & $\begin{array}{l}-134.7^{* * * *} \\
(9.915)\end{array}$ \\
\hline & & & & & & & & & & 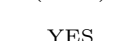 & \\
\hline $\begin{array}{l}\text { LAG } \\
\text { Observations }\end{array}$ & $\begin{array}{l}\mathrm{NO} \\
12,427\end{array}$ & No & $\begin{array}{l}\text { NO } \\
9880\end{array}$ & $\begin{array}{l}\text { YES } \\
12,240\end{array}$ & $\begin{array}{l}\text { YES } \\
2,529\end{array}$ & $\begin{array}{l}\text { YES } \\
2.528\end{array}$ & $\begin{array}{l}\text { YES } \\
5,057\end{array}$ & $\begin{array}{l}\text { YES } \\
4.677\end{array}$ & $\begin{array}{l}\text { YES } \\
7.183\end{array}$ & $\begin{array}{l}\text { YES } \\
12,427\end{array}$ & $\begin{array}{l}\text { YES } \\
12427\end{array}$ \\
\hline $\begin{array}{l}\text { Observations } \\
\text { Number of pais_numero }\end{array}$ & $\frac{12,427}{5}$ & $\frac{12,427}{5}$ & $\begin{array}{l}9,880 \\
4\end{array}$ & $\frac{12,240}{5}$ & $\begin{array}{l}2,529 \\
1\end{array}$ & $\begin{array}{l}2,528 \\
1\end{array}$ & $\begin{array}{c}5,057 \\
2\end{array}$ & $\frac{4,677}{2}$ & $\begin{array}{l}7,183 \\
3\end{array}$ & $\frac{12,427}{5}$ & $\begin{array}{l}12427 \\
5\end{array}$ \\
\hline R-squared & 0.018 & 0.027 & 0.091 & 0.029 & 0.221 & 0.224 & 0.202 & 0.128 & 0.043 & 0.027 & 0.027 \\
\hline
\end{tabular}

Robust standard errors in parenthese
$* * *$ pi $0.01, * * \mathrm{p} ; 0.05, *$ p 0.1

$*^{* *},{ }^{* *}, *$ : significance at the 1 percent, 5 percent and 10 percent levels. Robust standard errors in parenthesis.

Notes: (1) Limited subset of control variables; (2) All controls; (3) No Greece; (4) Introduction of 1 lag of the dependent variable; (5) Only Spain; (6) Only Italy; (7) Spain and Italy; (8) Ireland and Portugal; (9) Ireland, Portugal and Greece; (10) Events are only the financial-banking subset; (11) CMU decisions are added to the baseline decisions. Columns 1 to 9 include 26 decisions on financial and fiscal integration for the euro area as a whole; column 10 contains 19 decisions (only financial integration) and column 11 includes 45 decisions. 
Table 8: Results for the pool of peripheral and core euro area countries.

\begin{tabular}{|c|c|c|c|}
\hline VARIABLES & $\begin{array}{c}10 \text { year spread } \\
\text { (1) }\end{array}$ & $\begin{array}{c}5 \text { year spread } \\
(2)\end{array}$ & $\begin{array}{l}\text { OIS } \\
(3)\end{array}$ \\
\hline Decision t- $3 *$ periphery & $\begin{array}{c}2.546 \\
(1.746)\end{array}$ & $\begin{array}{c}4.523 \\
(2.792)\end{array}$ & $\begin{array}{c}4.315^{* * *} \\
(1.660)\end{array}$ \\
\hline Decision t-3 & $\begin{array}{c}0.375 \\
(0.870)\end{array}$ & $\begin{array}{c}0.610 \\
(1.582)\end{array}$ & $\begin{array}{c}-2.116^{* * *} \\
(0.804)\end{array}$ \\
\hline Decision $\mathrm{t}-2^{*}$ periphery & $\begin{array}{l}1.509 \\
(1.755)\end{array}$ & $\begin{array}{l}-2.316 \\
(2.833)\end{array}$ & $\begin{array}{c}0.390 \\
(1.673)\end{array}$ \\
\hline Decision t-2 & $\begin{array}{c}0.733 \\
(0.872)\end{array}$ & $\begin{array}{c}0.385 \\
(1.605)\end{array}$ & $\begin{array}{c}2.747^{* * *} \\
(0.807)\end{array}$ \\
\hline Decision $\mathrm{t}-1^{*}$ periphery & $\begin{array}{l}-0.854 \\
(1.763)\end{array}$ & $\begin{array}{l}5.013^{*} \\
(2.816)\end{array}$ & $\begin{array}{l}-0.752 \\
(1.680)\end{array}$ \\
\hline Decision t-1 & $\begin{array}{l}-0.195 \\
(0.876)\end{array}$ & $\begin{array}{c}0.406 \\
(1.608)\end{array}$ & $\begin{array}{l}-0.908 \\
(0.817)\end{array}$ \\
\hline Decision $t^{*}$ periphery & $\begin{array}{c}2.827 \\
(1.752)\end{array}$ & $\begin{array}{l}5.008^{*} \\
(2.815)\end{array}$ & $\begin{array}{l}3.487^{* *} \\
(1.672)\end{array}$ \\
\hline Decision $\mathrm{t}$ & $\begin{array}{c}0.805 \\
(0.870)\end{array}$ & $\begin{array}{c}0.438 \\
(1.603)\end{array}$ & $\begin{array}{l}-1.440^{*} \\
(0.812)\end{array}$ \\
\hline Decision $\mathrm{t}+1^{*}$ periphery & $\begin{array}{c}-5.021^{* * *} \\
(1.741)\end{array}$ & $\begin{array}{c}-16.49^{* * *} \\
(2.820)\end{array}$ & $\begin{array}{c}-5.116^{* * *} \\
(1.661)\end{array}$ \\
\hline Decision $\mathrm{t}+1$ & $\begin{array}{c}0.198 \\
(0.865)\end{array}$ & $\begin{array}{c}-0.0668 \\
(1.596)\end{array}$ & $\begin{array}{c}0.573 \\
(0.807)\end{array}$ \\
\hline Decision $t+2^{*}$ periphery & $\begin{array}{c}0.342 \\
(1.728)\end{array}$ & $\begin{array}{c}1.177 \\
(2.802)\end{array}$ & $\begin{array}{c}0.492 \\
(1.650)\end{array}$ \\
\hline Decision $t+2$ & $\begin{array}{l}-0.529 \\
(0.858)\end{array}$ & $\begin{array}{l}-0.316 \\
(1.586)\end{array}$ & $\begin{array}{c}0.217 \\
(0.802)\end{array}$ \\
\hline Decision $\mathrm{t}+3^{*}$ periphery & $\begin{array}{l}-0.0606 \\
(1.742)\end{array}$ & $\begin{array}{c}0.179 \\
(2.816)\end{array}$ & $\begin{array}{l}-0.181 \\
(1.661)\end{array}$ \\
\hline Decision $t+3$ & $\begin{array}{l}-0.391 \\
(0.859)\end{array}$ & $\begin{array}{l}-0.394 \\
(1.568)\end{array}$ & $\begin{array}{l}-0.361 \\
(0.801)\end{array}$ \\
\hline Constant & $\begin{array}{l}-6.338 \\
(5.187)\end{array}$ & $\begin{array}{l}-2.558 \\
(9.334)\end{array}$ & $\begin{array}{c}1.393 \\
(4.799)\end{array}$ \\
\hline LAG & $\mathrm{NO}$ & $\mathrm{NO}$ & NO \\
\hline Observations & 17,496 & 16,657 & 20,165 \\
\hline Countries & 7 & 7 & 8 \\
\hline R-squared & 0.030 & 0.051 & 0.027 \\
\hline
\end{tabular}

***, **, *: significance at the 1 percent, 5 percent and 10 percent levels. Robust standard errors in parenthesis.

Notes: The decisions included are the 39 main financial and fiscal integration decisions. All regressions include the complete set of controls and their interactions. Periphery takes value 1 if the country is Spain, Italy, Portugal, Ireland or Greece and 0 if it is Germany, France or The Netherlands. In columns 1 and 2, the interaction coefficient measures the difference between the movements of the periphery spreads with respect to Germany against the movements of the spreads of France and the Netherlands with respect to Germany. In column 3, it measures the difference between the movements of the periphery spreads with respect to the OIS against the movements of the spreads of France, Germany and the Netherfands with respect to the OIS. 


\section{References}

Afonso, A., Arghyrou, M. G., Bagdatoglou, G., and Kontonikas, A. (2015). On the timevarying relationship between emu sovereign spreads and their determinants. Economic Modelling, 44:363-371.

Afonso, A., Jalles, J. T., and Kazemi, M. (2020). The effects of macroeconomic, fiscal and monetary policy announcements on sovereign bond spreads. International Review of Law and Economics, Article 105924.

Ait-Sahalia, Y., Andritzky, J., Jobst, A., Nowak, S., and Tamirisa, N. (2012). Market response to policy initiatives during the global financial crisis. Journal of International Economics, 87(1):162-177.

Aizenman, J., Hutchison, M., and Jinjarak, Y. (2013). What is the risk of european sovereign debt defaults? fiscal space, cds spreads and market pricing of risk. Journal of International Money and Finance, 34:37-59.

Altavilla, C., Giannone, D., and Lenza, M. (2014). The financial and macroeconomic effects of omt announcements. ECB working paper, (1707).

Arghyrou, M. G. and Kontonikas, A. (2012). The emu sovereign-debt crisis: Fundamentals, expectations and contagion. Journal of International Financial Markets, Institutions and Money, 22(4):658-677.

Bahaj, S. (2020). Sovereign spreads in the euro area: Cross border transmission and macroeconomic implications. Journal of Monetary Economics, 110:116-135.

Bank of Spain (2020). Next generation eu: main characteristics and impact of its announcement on financial conditions'. Economic Bulletin, Box 5, 3/2020.

Barbosa, L., Costa, S., et al. (2010). Determinants of sovereign bond yield spreads in the euro area in the context of the economic and financial crisis. Banco de Portugal Working Paper, 22/2010.

Barrios, S., Iversen, P., Lewandowska, M., Setzer, R., et al. (2009). Determinants of intraeuro area government bond spreads during the financial crisis. Directorate General Economic and Financial Affairs (DG ECFIN), European Commission. 
Beetsma, R., Giuliodori, M., De Jong, F., and Widijanto, D. (2013). Spread the news: The impact of news on the european sovereign bond markets during the crisis. Journal of International Money and finance, 34:83-101.

Beirne, J. and Fratzscher, M. (2013). The pricing of sovereign risk and contagion during the european sovereign debt crisis. Journal of International Money and Finance, 34:60-82.

Bergman, U. M., Hutchison, M. M., and Jensen, S. E. H. (2019). European policy and markets: Did policy initiatives stem the sovereign debt crisis in the euro area? European Journal of political economy, 57:3-21.

Bhanot, K., Burns, N., Hunter, D., and Williams, M. (2012). Was there contagion in eurozone sovereign bond markets during the greek debt crisis. The University of Texas at San Antonio Working Paper Series 006FIN-73-2012.

Costantini, M., Fragetta, M., and Melina, G. (2014). Determinants of sovereign bond yield spreads in the emu: An optimal currency area perspective. European Economic Review, 70:337-349.

De Grauwe, P. (2011). Managing a fragile eurozone. In CESifo Forum, volume 12, pages 40-45. München: ifo Institut-Leibniz-Institut für Wirtschaftsforschung an der Universität München.

De Grauwe, P. and Ji, Y. (2012). Mispricing of sovereign risk and macroeconomic stability in the eurozone. JCMS: Journal of Common Market Studies, 50(6):866-880.

Dewachter, H., Iania, L., Lyrio, M., and de Sola Perea, M. (2015). A macro-financial analysis of the euro area sovereign bond market. Journal of Banking \& Finance, 50:308-325.

Georgoutsos, D. and Migiakis, P. (2018). Risk perceptions and fundamental effects on sovereign spreads. Bank of Greece Working Papers, 250.

Gerlach, S., Schulz, A., and Wolff, G. B. (2010). Banking and sovereign risk in the euro area. Bundesbank Series 1 Discussion Paper, (9).

Gros, D. (2018). Italian risk spread: Fiscal versus redenomination risk. VoxEU. org, 29.

Krishnamurthy, A., Nagel, S., and Vissing-Jorgensen, A. (2018). Ecb policies involving government bond purchases: Impact and channels. Review of Finance, 22(1):1-44. 
Mink, M. and De Haan, J. (2013). Contagion during the greek sovereign debt crisis. Journal of International Money and Finance, 34:102-113.

Missio, S. and Watzka, S. (2011). Financial contagion and the european debt crisis. CESifo Working Paper Series, (3554).

Mohl, P. and Sondermann, D. (2013). Has political communication during the crisis impacted sovereign bond spreads in the euro area? Applied Economics Letters, 20(1):48-61.

Poghosyan, T. (2014). Long-run and short-run determinants of sovereign bond yields in advanced economies. Economic Systems, 38(1):100-114.

Pynnönen, S. (2005). On regression based event study. Acta Wasaensia, 143(2):327-354.

Rivolta, G. (2014). An event study analysis of ecb unconventional monetary policy. Universita degli Studi di Milano Departmental Working Papers, 2.

Saka, O., Fuertes, A.-M., and Kalotychou, E. (2015). ECB policy and eurozone fragility: Was de grauwe right? Journal of International Money and Finance, 54:168-185.

Sgherri, S. and Zoli, E. (2009). Euro area sovereign risk during the crisis. IMF Working Papers, WP/09/222, pages 1-23.

Von Hagen, J., Schuknecht, L., and Wolswijk, G. (2011). Government bond risk premiums in the eu revisited: The impact of the financial crisis. European Journal of Political Economy, $27(1): 36-43$. 


\section{Annex A: Detailed description of the narrative}

In this section, we attempt to provide a description for each topic collected from the official reports after meetings of the European Council and the Council of the European Union (includes ECOFIN and Eurogroup) between February 11, 2010 and March 30, 2020. This will allow to present clear-cut evidence behind our conclusions regarding the effect of the statements of these institutions on the behavior of the 5 and 10 years sovereign bonds in the Eurozone member sates. Some of those meetings or documents considered as decision in this section will not be included in the final list of decisions due to the fact that they are minor modifications; ie. reviews in bailout programs (decision 6 and 8 in the Greek narrative, A.1).

Topics are classified into three typologies according to their subject. On the one hand, we have those matters related to Crisis Management and Fiscal Issues, which are events that affect disproportionately one single country, and, on the other hand, Economic Integration events, which are those events that have a significant impact on the European block as a whole. Each topic is followed up over time, highlighting the date on which a first statement is made and the date when a decision is made on the topic. A first statement is considered to be any statement that shows a clear intention to deal with an issue in depth (to mention the purpose to develop a legislative project, the creation of a commission, the time when a work team mentions its intention to move forward on a specific issue, willingness to accept requests for fiscal aid from a member country, etc.). A decision is considered to be a statement on a specific measure or a legislative change for which there is an agreement; this announcement prevails as a decision either if it occurs before the date of implementation of the measure or before the agreement is signed. Note that there may be several first statement entries for the same topic as a consequence of a review of the issue (a review also introduces possible hesitation about the continuity without changes of a bailout program). Likewise, there may also be several decision entries for the same topic, one for each significant change or closure of an issue that has been reopened. The set of statements between the first statement and the document previous to the final decision, those that served to prepare the first statement and those after the decision that only inform about the accomplishment of agreed terms constitute the monitoring group.

\section{A.1 Greece}

Decision 1 on Greece was taken by the Eurogroup in May 2, 2010 stating that "[...] euro area Ministers unanimously agreed today to activate stability support to Greece via bilateral 
loans centrally pooled by the European Commission under the conditions set out in their statement of 11 April." The first statement 1 regarding Greece corresponds to the Eurogroup meeting of April 11, 2010 where they declared that "[...] Euro area Members States have agreed upon the terms of the financial support that will be given to Greece, when needed, to safeguard financial stability in the Euro area as a whole." The monitoring group 1 for Greece comprises European Council statements of February 11, 2010; March 25, 2010 and April 23, 2010; and Eurogroup statements of November 16, 2010 (considered a fiscal issue), July 2, 2011 and October 21, 2011. Decision 2 on Greece was stated on the Euro Summit of October 26, 2011 where they declared that "Together with an ambitious reform program for the Greek economy, the PSI (Private Sector Involvement) should secure the decline of the Greek debt to GDP ratio with an objective of reaching $120 \%$ by 2020 . To this end we invite Greece, private investors and all parties concerned to develop a voluntary bond exchange with a nominal discount of $50 \%$ on notional Greek debt held by private investors. The Eurozone Member States would contribute to the PSI package up to 30 bn euro. On that basis, the official sector stands ready to provide additional program financing of up to 100 bn euro until 2014, including the required recapitalization of Greek banks." The first statement 2 regarding Greece corresponds to the Eurogroup meeting of June 20, 2011 where they declared that "On these conditions, Ministers decided to define by early July the main parameters of a clear new financing strategy." The monitoring group 2 for Greece comprises the European Council statement of June 24, 2011 and the Euro Summit of July 21, 2011. Decision 3 on Greece was taken by the Eurogroup in November 27, 2012 stating that "The Eurogroup recalls that a full staff-level agreement has been reached between Greece and the Troika on updated program conditionality [...]. The Eurogroup noted with satisfaction that the updated program conditionality includes the adoption by Greece of new instruments to enhance the implementation of the program, notably by means of correction mechanisms to safeguard the achievement of both fiscal and privatization targets, and by stronger budgeting and monitoring rules." The first statement 3 regarding Greece corresponds to the European Council meeting of January 30, 2012 where they declared that "Concerning Greece, we note progress made in the negotiations with the private sector to reach an agreement in line with the parameters agreed upon in October. We urge the Greek authorities and all parties involved to finalize negotiations on the new program in the coming days." Decision 4 on Greece was taken by the Eurogroup in June 27, 2015 stating that "Regrettably, despite efforts at all levels and full support of the Eurogroup, this proposal has been rejected by the 
Greek authorities who broke off the program negotiations late on the 26 June unilaterally." The first statement 4 regarding Greece corresponds to the Eurogroup meeting of February 20, 2015 where they declared that "The Eurogroup notes, in the framework of the existing arrangement, the request from the Greek authorities for an extension of the Master Financial Assistance Facility Agreement (MFFA), which is underpinned by a set of commitments." The monitoring group 4 for Greece comprises Eurogroup statements of February 24, 2015; April 24, 2015; May 11, 2015; June 18, 2015; June 24, 2015; and June 25, 2015; the Euro Summit of May 16, 2015; the European Council statement of June 26, 2015; and the Council of the European Union statement of March 20, 2015. Decision 5 on Greece was taken by the Eurogroup in August 14, 2015 stating that "The Eurogroup welcomes the agreement that has been reached between Greece and the European Institutions, with input from the IMF, on the policy conditionality underlying the new ESM macroeconomic adjustment program. [...] The Eurogroup welcomes the wide scope of the policy measures contained in the Memorandum of Understanding (MoU), which, if implemented with determination, will address the main challenges facing the Greek economy." The first statement 5 regarding Greece corresponds to the Euro Summit of July 12, 2015 where they declared that "The Euro Summit stresses the crucial need to rebuild trust with the Greek authorities as a prerequisite for a possible future agreement on a new ESM program. [...] Given the need to rebuild trust with Greece, the Euro Summit welcomes the commitments of the Greek authorities to legislate without delay a first set of measures." The monitoring group 5 for Greece comprises Eurogroup statements of July 13, 2015; July 16, 2015; July 17, 2015; August 19, 2015; October 5, 2015; November 9, 2015; November 17, 2015; November 21, 2015; November 23, 2015; December 7, 2015; January 14, 2016; April 22, 2016; May 9, 2016; May 25, 2016; September 9, 2016; October 10, 2016; and November 7, 2016. Decision 6 on Greece was taken by the Eurogroup in June 15, 2017 stating that "We welcomed the ambitious policy package that was agreed between Greece and the institutions and the satisfactory adoption of the agreed prior actions for the second review. [...] We addressed the ESM proposal for the terms of the third tranche and signaled political agreement thereto." The first statement 6 regarding Greece corresponds to the Eurogroup meeting of December 5, 2016 where they declared that "In order to ensure compliance with the fiscal targets in a sustainable manner after the completion of the program, the Greek authorities commit to agree with the institutions on a mechanism and structural measures that would ensure this." The monitoring group 6 for Greece comprises Eurogroup statements of January 26, 2017; 
February 20, 2017; March 20, 2017; April 7, 2017; May 22, 2017; and September 15, 2017. Decision 7 on Greece was taken by the Council of the European Union in September 25, 2017, as part of the fiscal decision subset stating that "The Council has closed the excessive deficit procedure for Greece. It confirmed that the country's deficit is now below $3 \%$ of GDP, the EU's reference value for government deficits." Decision 8 on Greece was taken by the Eurogroup in March 12, 2018 stating that "The European institutions debriefed the Eurogroup on the closure of the third review and on the state of play of the fourth review of the ESM stability support program. We welcomed that Greece has finalized the third review, which will pave the way for the first disbursement of the fourth tranche after the finalization of national procedures expected in the second half of March." The first statement 8 regarding Greece corresponds to the Eurogroup meeting of November 6, 2017 where they declared that "The institutions debriefed us on their recent mission to Greece in the context of the third review. [...] The objective remains to complete the third review of the ESM program ideally by the end of 2017 . The Eurogroup will have a more in depth discussion at the December meeting." The monitoring group 8 for Greece comprises Eurogroup statements of December 4, 2017, February 19, 2018 and March 2, 2018. Decision 9 on Greece was taken by the Eurogroup in June 21, 2018 stating that "We welcomed the confirmation by the Institutions that the Greek authorities have completed all the prior actions under the fourth and final review. This allowed us to conclude that Greece has successfully completed the ESM program. We agreed on a statement that welcomes the post program commitments by the Greek authorities and lays out the agreed debt measures and the final disbursement [...]." The first statement 9 regarding Greece corresponds to the Eurogroup meeting of April 27, 2018 where they declared that "The institutions debriefed the Eurogroup on the state of play of the fourth review of the ESM stability support program and announced the return of the mission teams to Athens on 14 May. The aim is to reach a staff level agreement ahead of the May Eurogroup. We also held an exchange of views on some key elements to support the successful exit of Greece from the program." The monitoring group 9 for Greece comprises the Eurogroup statement of May 24, 2018. Decision 10 on Greece was taken by the Eurogroup in April 5, 2019 stating that "We were informed by the European Institutions that Greece has taken the necessary actions to achieve all specific reform commitments for end 2018 and that the necessary conditions are in place to confirm the release of the first tranche of policy-contingent debt measures." The monitoring group 10 for Greece comprises the Eurogroup statement of December 4, 2019. 


\section{A.2 EFSM}

Decision 1 on EFSM was taken by the Council of the European Union in May 9, 2010 stating that "The Council and the Member States have decided today on a comprehensive package of measures to preserve financial stability in Europe, including a European Financial Stabilization mechanism with a total volume of up to EUR 500 billion." The first statement 1 regarding EFSM corresponds to the European Council meeting of May 7, 2010 where they declared that "[...] taking into account the exceptional circumstances, the Commission will propose a European stabilization mechanism to preserve financial stability in Europe." Decision 2 on EFSM was taken by the Council of the European Union in August 4, 2015 stating that "[...] the Council approved a regulation amending the European Financial Stabilization Mechanism (EFSM) in order to protect non-euro area member states from any risk arising from financial assistance given to a euro area country."

\section{A.3 EFSF}

Decision 1 on EFSF was taken by the Eurogroup in June 7, 2010 stating that "In line with the decisions taken on 9 May within the framework of the ECOFIN Council to safeguard financial stability in Europe, Ministers have established the European Financial Stability Facility as a limited liability company under Luxembourg law (Société Anonyme). [...] The objective of the EFSF is to collect funds and provide loans in conjunction with the IMF to cover the financing needs of euro area Member States in difficulty, subject to strict policy conditionality." The first statement 1 regarding EFSF corresponds to the Council of the European Union meeting of May 9, 2010 where they declared that "[...] euro area Member States stand ready to complement such resources through a Special Purpose Vehicle that is guaranteed on a pro rata basis by participating Member States in a coordinated manner and that will expire after three years, respecting their national constitutional requirements, up to a volume of EUR 440 billion." Decision 2 on EFSF was taken by the European Council in March 11, 2011 stating that "[...] Pricing of the EFSF should be lowered to better take into account debt sustainability of the recipient countries, while remaining above the funding costs of the facility, with an adequate mark up for risk, and in line with the IMF pricing principles." The first statement 2 regarding EFSF corresponds to the European Council meeting of February 4, 2011 where they declared that "[...] they agreed on the following steps as part of the global package to be finalized in March: [... Concrete proposals by the Eurogroup on the strengthening of the EFSF so as to ensure the necessary effectiveness to provide adequate 
support." The monitoring group 2 for EFSF comprises the European Council statement of June 24, 2011. Decision 3 on EFSF was stated on the Euro Summit of July 21, 2011 where they declared that "To improve the effectiveness of the EFSF and of the ESM and address contagion, we agree to increase their flexibility linked to appropriate conditionality, allowing them to: act on the basis of a precautionary program; finance recapitalization of financial institutions through loans to governments including in non-program countries; intervene in the secondary markets on the basis of an ECB analysis recognizing the existence of exceptional financial market circumstances and risks to financial stability and on the basis of a decision by mutual agreement of the EFSF/ESM Member States, to avoid contagion." The first statement 3 regarding EFSF corresponds to the Eurogroup meeting of July 11, 2011 where they declared that "[...] Ministers stand ready to adopt further measures that will improve the euro area's systemic capacity to resist contagion risk, including enhancing the flexibility and the scope of the EFSF, lengthening the maturities of the loans and lowering the interest rates, including through a collateral arrangement where appropriate. Proposals to this effect will be presented to Ministers shortly." The monitoring group 3 for EFSF comprises the Euro Summits of October 26, 2011 and December 9, 2011. Decision 4 on EFSF was taken by the Eurogroup on July 9, 2012 stating that "[...] the ECB and the EFSF have signed today a technical agency agreement creating the possibility of an efficient conduct of market operations by the EFSF. As soon as the ESM has been established, a similar agreement will be concluded between the ECB and ESM." Decision 5 on EFSF was taken by the Eurogroup in March 30, 2012 stating that "[...] The EFSF will, as a rule, only remain active in financing programs that have started before that date. For a transitional period until mid-2013, it may engage in new programs in order to ensure a full fresh lending capacity of EUR 500 billion. The current overall ceiling for ESM/EFSF lending, as defined in the ESM Treaty, will be raised to EUR 700 billion such that the ESM and the EFSF will be able to operate, if needed, as described above."

\section{A.4 Excessive Deficit}

Decision 1 on Excessive Deficit was taken by the Council of the European Union in July 13, 2010 stating that "The Council also opened excessive deficit procedures in relation to Bulgaria, Cyprus, Denmark and Finland, making recommendations on the measures to be taken to reduce their deficits to below the reference value of $3 \%$ of gross domestic product." Decision 2 on Excessive Deficit was taken by the Eurogroup in November 16, 2010 stating 
that "Based on methodology fully in line with European standards, there is, as expected, a substantial revision in government deficit and debt data for 2009". Decision 3 on Excessive Deficit was taken by the Council of the European Union in July 12, 2011 stating that "The Council also decided to close the excessive deficit procedure with regard to Finland, concluding that its excessive deficit has been corrected." Decision 4 on Excessive Deficit was taken by the Council of the European Union in June 22, 2012 stating that "The Council adopted decision closing excessive deficit procedures for Germany and Bulgaria, thus confirming that they have reduced their deficits below $3 \%$ of GDP, the EU's reference value for government deficits." Decision 5 on Excessive Deficit was taken by the Eurogroup in October 9, 2012 stating that "The Council adopted decision modifying the conditions underpinning financial assistance to Portugal, granting it an additional year, until 2014, to correct its excessive deficit." Decision 6 on Excessive Deficit was taken by the Council of the European Union in June 21, 2013 stating that "The Council closed excessive deficit procedures for Italy, Latvia, Lithuania, Hungary and Romania [...]." Decision 7 on Excessive Deficit was taken by the Council of the European Union in June 21, 2013 stating that "[... extended the deadlines for Spain, France, the Netherlands, Poland, Portugal and Slovenia to correct their deficits [...]." Decision 8 on Excessive Deficit was taken by the Council of the European Union in January 28, 2014 stating that "The Council adopted a decision establishing the existence of an excessive government deficit in Croatia." Decision 9 on Excessive Deficit was taken by the Council of the European Union in June 20, 2014 stating that "The Council closed excessive deficit procedures for Belgium, the Czech Republic, Denmark, the Netherlands, Austria and Slovakia, confirming that these countries have reduced their deficits below $3 \%$ of GDP, the EU's reference value for government deficits." Decision 10 on Excessive Deficit was taken by the Council of the European Union in March 10, 2015 stating that "The Council granted France two extra years to bring its government deficit below $3 \%$ of GDP, the reference value for deficits set by the EU's Stability and Growth Pact." Decision 11 on Excessive Deficit was taken by the Council of the European Union in June 19, 2015 stating that "The Council closed excessive deficit procedures for Malta and Poland [...]." Decision 12 on Excessive Deficit was taken by the Council of the European Union in July 12, 2015 stating that "The Council on 13 July 2015 imposed on Spain a fine of EUR 18.93 million for the manipulation of deficit data in the Autonomous Community of Valencia (Comunidad Valenciana)." Decision 13 on Excessive Deficit was taken by the Council of the European Union in June 17, 2016 stating that "The Council closed excessive deficit procedures for Cyprus, Ireland and 
Slovenia." Decision 14 on Excessive Deficit was taken by the Council of the European Union in July 12, 2016 stating that "The Council adopted decisions establishing that Portugal and Spain had not taken effective action in response to its recommendations on measures to correct their excessive deficits. [...] The Council's decisions will trigger sanctions under article 126(8) of the Treaty on the Functioning of the European Union." The first statement 14 regarding Excessive Deficit corresponds to the Eurogroup meeting of July 11, 2016 where they declared that "The Commission presented its recommendations to the Council under article 126.8 TFEU establishing that no effective action has been taken by Spain and Portugal to correct their excessive deficits. It also clarified the next steps under the SGP procedure. If the Council adopts the two decisions on lack of effective action the following day, only then the various steps of the sanctions procedures would be triggered." Decision 15 on Excessive Deficit was taken by the Council of the European Union in August 9, 2016 stating that "[...] the Council agreed not to impose fines on Portugal and Spain for their failure to take effective action to correct their excessive deficits. It also stepped up the excessive deficit procedure for both countries, setting new correction deadlines and giving notice of measures to be taken." Decision 16 on Excessive Deficit was taken by the Council of the European Union in June 16, 2017 stating that "It closed excessive deficit procedures for Croatia and Portugal, confirming they have reduced their deficits below $3 \%$ of GDP, the EU's reference value for government deficits." Decision 17 on Excessive Deficit was taken by the Council of the European Union in September 25, 2017 stating that "The Council has closed the excessive deficit procedure for Greece[...]." Decision 18 on Excessive Deficit was taken by the Council of the European Union in December 5, 2017 stating that "Abrogating its 2008 decision on the existence of an excessive deficit in the UK, the Council confirmed that the UK has reduced its government deficit to below 3\% of GDP, the EU's reference value for government deficits." Decision 19 on Excessive Deficit was taken by the Council of the European Union in June 22, 2018 stating that "It closed the excessive deficit procedure for France, confirming that it has reduced its deficit below 3\% of GDP, the EU's reference value for government deficits." Decision 20 on Excessive Deficit was taken by the Council of the European Union in June 14, 2019 stating that "The Council closed the excessive deficit procedure for Spain, confirming that it has reduced its deficit below the EU's 3\% of GDP reference value. The Council thereby abrogated its decision of April 2009 on the existence of an excessive deficit in Spain. As a consequence, no member state remains subject to an excessive deficit procedure. Procedures were open for 24 member states in 2010-11 at the height of the euro crisis." 


\section{A.5 European Semester}

Decision 1 on European Semester was taken by the European Council in October 21, 2010 stating that "One of the earliest Task Force recommendations to reinforce policy coordination, the so-called European Semester, has already been decided and will be implemented as of 1st January 2011. Each spring, it will allow a simultaneous assessment of both budgetary measures and structural reforms fostering growth and employment. This will contribute to ensure that the EU/euro area dimension is better taken into account when countries prepare budgets and reform programs." The first statement 1 regarding European Semester corresponds to the Council of the European Union meeting of September 7, 2010 where they declared that "The Council also endorsed changes to the manner in which the EU's stability and growth pact is implemented in order to allow a "European semester" to be introduced, as from 2011, as part of a reform of EU provisions on economic policy coordination." The monitoring group 1 for European Semester comprises the Council of the European Union statement of July 13, 2010.

\section{A.6 Supervision of the Financial System}

Decision 1 on Supervision of the Financial System was taken by the Council of the European Union in November 17, 2010 stating that "The Council adopted legal texts underpinning a reform of the EU framework for supervision of the financial system, aimed at eliminating deficiencies that were exposed during the financial crisis. It adopted regulations establishing: a European Systemic Risk Board (ESRB), which will provide macro-prudential oversight of the financial system (39/10); a European Banking Authority (EBA) for the supervision of banking services (doc. 40/10); a European Insurance and Occupational Pensions Authority (EIOPA) for the supervision of insurance services (41/10); a European Securities and Markets Authority (ESMA) for the supervision of the securities industry (42/10)." The first statement 1 regarding Supervision of the Financial System corresponds to the Council of the European Union meeting of July 13, 2010 where they declared that "The Council adopted a political guideline for continuing negotiations with the European Parliament on the reform of financial supervision in Europe." The monitoring group 1 for Supervision of the Financial System comprises the Council of the European Union statement of September 7, 2010. Decision 2 on Supervisory Framework for Financial Institutions was taken by the Council of the European Union in December 2, 2019 stating that "The EU will have an improved supervisory framework for European financial institutions in place by the end of the year. 
The Council adopted today a first fundamental review of the functioning of the European system of financial supervision (ESFS)."

\section{A.7 Ireland}

Decision 1 on Ireland was taken by the Council of the European Union in November 28, 2010 stating that "Ministers unanimously agreed today to grant financial assistance in response to the Irish authorities' request on November 22, 2010." The first statement 1 regarding Ireland corresponds to the Council of the European Union meeting of November 21, 2010 where they declared that "Ministers welcome the request of the Irish Government for financial assistance from the European Union and euro-area Member States. Ministers concur with the Commission and the ECB that providing assistance to Ireland is warranted to safeguard financial stability in the EU and in the euro area." The monitoring group 1 for Ireland comprises the Eurogroup statement of November 16, 2010. Decision 2 on Ireland was taken by the Council of the European Union in April 12, 2013 stating that "The Eurogroup and ECOFIN Ministers discussed today the state of play of the adjustment programs in Ireland and in Portugal. [...] To support their efforts to regain full market access and successfully exit their programs, we agreed in principle, subject to national procedures, to lengthen the maturities of the EFSM and EFSF loans to Ireland and Portugal by increasing the weighted average maturity limit by 7 years [...]." The first statement 2 regarding Ireland corresponds to the Eurogroup meeting of March 16, 2013 where they declared that "The Eurogroup ministers are determined to support Ireland's and Portugal's efforts to regain full market access and successfully exit their well-performing programs, in the context of continued strong program implementation and compliance. They have agreed to an adjustment of the maturities of the EFSF loans to both countries in order to smooth the debt redemption profiles of those countries. The technical details will be put forward [...]." The monitoring group 2 for Ireland comprises Eurogroup statements of October 14, 2013; November 14, 2013 and December 9, 2013.

\section{A.8 Article 136}

Decision 1 on Article 136 was taken by the European Council in March 25, 2011 stating that "The European Council has decided to add to Article 136 of the Treaty the following paragraph: "The Member States whose currency is the euro may establish a stability mechanism to be activated if indispensable to safeguard the stability of the euro area as a whole. 
The granting of any required financial assistance under the mechanism will be made subject to strict conditionality"." The first statement 1 regarding Article 136 corresponds to the Eurogroup meeting of March 15, 2010 where they declared that "[... ] the Eurogroup stands ready to explore the possibilities of Article 136 of the new Treaty as a basis for clearer and more ambitious recommendations and measures addressed to the euro area Member States to ensure a smooth functioning of EMU." The monitoring group 1 for Article 136 comprises the European Council statement of March 26, 2010.

\section{A.9 Euro Plus Pact}

Decision 1 on Euro Plus Pact was taken by the European Council in March 25, 2011 stating that "The Euro Plus Pact as agreed by the euro area Heads of State or government and joined by Bulgaria, Denmark, Latvia, Lithuania, Poland, Romania (see annex I) will further strengthen the economic pillar of EMU and achieve a new quality of economic policy coordination, with the objective of improving competitiveness and thereby leading to a higher degree of convergence reinforcing our social market economy." The first statement 1 regarding Euro Plus Pact corresponds to the European Council meeting of March 11, 2011 where they declared that "The Pact for the Euro which establishes a stronger economic policy coordination for competitiveness and convergence (attached) has been endorsed. This Pact will be presented to the European Council of 24/25 March 2011 with a view for non-euro area Member States to indicate whether they intend to participate in the Pact. At the same time Euro area Member States shall indicate first measures they pledge to implement under the Pact for the next year." The monitoring group 1 for Euro Plus Pact comprises the Council of the European Union statement of November 30, 2011.

\section{A.10 Portugal}

Decision 1 on Portugal was taken by the Council of the European Union in May 16, 2011 stating that "Euro area and EU financial support will be provided on the basis of a program which has been negotiated with the Portuguese authorities by the Commission, in liaison with the ECB, and the IMF." The first statement 1 regarding Portugal corresponds to the Council of the European Union meeting of April 8, 2011 where they declared that "Ministers acknowledged the Portuguese authorities' request for financial assistance. Ministers invited the Commission, the ECB, the IMF and Portugal to set up a program and take appropriate action to safeguard financial stability." The monitoring group 1 for Portugal comprises 
Eurogroup statements of September 30, 2010; November 16, 2010; September 14, 2012 and October 8, 2012. Decision 2 on Portugal was taken by the Eurogroup in October 9, 2012 stating that "The Council adopted decision modifying the conditions underpinning financial assistance to Portugal, granting it an additional year, until 2014, to correct its excessive deficit." Decision 3 on Portugal was taken by the Council of the European Union in April 12, 2013 stating that "The Eurogroup and ECOFIN Ministers discussed today the state of play of the adjustment programs in Ireland and in Portugal. [...] To support their efforts to regain full market access and successfully exit their programs, we agreed in principle, subject to national procedures, to lengthen the maturities of the EFSM and EFSF loans to Ireland and Portugal by increasing the weighted average maturity limit by 7 years." The first statement 3 regarding Portugal corresponds to the Eurogroup meeting of March 16, 2013 where they declared that "The Eurogroup ministers are determined to support Ireland's and Portugal's efforts to regain full market access and successfully exit their well-performing programs, in the context of continued strong program implementation and compliance. They have agreed to an adjustment of the maturities of the EFSF loans to both countries in order to smooth the debt redemption profiles of those countries. The technical details will be put forward to the Eurogroup by the Troika [...]." The monitoring group 3 for Portugal comprises Eurogroup statements of April 1, 2014 and May 5, 2014. Decision 4 on Portugal was taken by the Council of the European Union in June 12, 2014 stating that "We take note that the government has decided not to present replacement measures until concrete views on the constitutionality of such measures are obtained from the Constitutional Court, therefore forgoing the disbursement of the remaining EUR 1.7 bn of EFSM funds available until the end of June."

\section{A.11 ESM}

Decision 1 on ESM was taken by the Eurogroup in July 11, 2011 stating that "On July 11, 2011, a first Treaty establishing the European Stability Mechanism was signed in Brussels by the 17 Member States in the euro area." The first statement 1 regarding ESM corresponds to the European Council meeting of October 29, 2010 where they declared that "Further

to the report of the Task Force, and in order to ensure balanced and sustainable growth, Heads of State or Government agree on the need for Member States to establish a permanent crisis mechanism to safeguard the financial stability of the euro area as a whole and invite the President of the European Council to undertake consultations with the members 
of the European Council on a limited treaty change required to that effect, not modifying article 125 TFEU (no bail-out clause)." The monitoring group 1 for ESM comprises the Eurogroup statement of November 28, 2010; the Council of the European Union declaration of December 7, 2010; and the European Council statements of December 17, 2010; February 4, 2011; March 11, 2011 and March 25, 2011. Decision 2 on ESM was taken by the European Council in January 30, 2012 stating that "The Treaty establishing the European Stability Mechanism is ready for signature, and the objective is that it enters into force in July 2012. This permanent crisis mechanism will contribute to raising confidence, solidarity and financial stability in the euro area. It will have a wide range of tools available and a strong financial basis." The first statement 2 regarding ESM corresponds to the Euro Summit of December 9, 2011 where they declared that "We agree on the following adjustments to the ESM Treaty to make it more effective: Concerning the involvement of the private sector, we will strictly adhere to the well-established IMF principles and practices. [...] Concerning the involvement of the private sector, we will strictly adhere to the well-established IMF principles and practices [...]." The monitoring group 2 for ESM comprises the Euro Summit of September 27, 2012; the Eurogroup statement of February 11, 2013; and the European Council statements of February 2, 2012 and March 2, 2012. Decision 3 on ESM was taken by the Eurogroup in June 20, 2013 stating that "ESM direct bank recapitalization instrument. Main features of the operational framework and way forward." The first statement 3 regarding ESM corresponds to the European Council meeting of October 19, 2012 where they declared that "The Eurogroup will draw up the exact operational criteria that will guide direct bank recapitalizations by the European Stability Mechanism [...]." The monitoring group 3 for ESM comprises the European Council statement of December 14, 2012. Decision 4 on ESM was taken by the Eurogroup in June 10, 2014 stating that "Euro area Member States have today reached a political understanding on the operational framework of the ESM direct recapitalization instrument, [...] The instrument may be activated in case a bank fails to attract sufficient capital from private sources and if the ESM Member concerned is unable to recapitalize it, [...]." The first statement 4 regarding ESM corresponds to the Eurogroup meeting of February 17, 2014 where they declared that "we have reviewed the operational framework of the future ESM direct recap instrument. We have made progress towards resolving the last outstanding issues and I am confident that we will come to a political agreement in time for our meeting in March." The monitoring group 4 for ESM comprises the Eurogroup statement of May 5, 2014. Decision 5 on ESM was taken 
by the Eurogroup in December 4, 2018 stating that "We agreed on a term sheet, annexed to this statement, for the further development of the ESM." The monitoring group 5 for ESM comprises the Eurogroup statements of June 15, 2019, March 16, 2020 and March 24, 2020; and the Euro Summit of June 21, 2019.

\section{A.12 Six-Pack}

Decision 1 on Six-Pack was taken by the Council of the European Union in October 4, 2011 stating that "The Council also approved the text recently voted by the European Parliament on a package of six legislative measures designed to strengthen economic governance in the EU." The first statement 1 regarding Six-Pack corresponds to the European Council meeting of October 29, 2010 where they declared that "The European Council endorses the report of the Task Force on economic governance. Its implementation will allow us to increase fiscal discipline, broaden economic surveillance, deepen coordination, and set up a robust framework for crisis management and stronger institutions. The European Council calls for a fast track approach to be followed on the adoption of secondary legislation needed for the implementation of many of the recommendations." The monitoring group 1 for Six-Pack comprises European Council statements of December 17, 2010, February 4, 2011, March 11, 2011 and March 25, 2011; Council of the European Union statements of February 15, 2011, March 15, 2011, May 17, 2011 and June 20, 2011; the Eurogroup statement of July 11, 2011; and Euro Summits of July 21, 2011 and September 28, 2011.

\section{A.13 Fiscal Compact}

Decision 1 on Fiscal Compact was taken by the European Council in January 30, 2012 stating that "The Treaty on stability, coordination and governance in the Economic and Monetary Union has been finalized. It will be signed in March." The monitoring group 1 for Fiscal Compact comprises the European Council statement of March 2, 2012.

\section{A.14 Spain}

Decision 1 on Spain was taken by the Eurogroup in July 20, 2012 stating that "Ministers unanimously agreed today to grant financial assistance for the recapitalization of financial institutions in response to the Spanish authorities' request on 25 June 2012." The first statement 1 regarding Spain corresponds to the Eurogroup meeting of June 9, 2012 where they declared that "The Eurogroup has been informed that the Spanish authorities will 
present a formal request shortly and is willing to respond favorably to such a request. The financial assistance would be provided by the EFSF/ESM for recapitalization of financial institutions." The monitoring group 1 for Spain comprises the Eurogroup statements of June 27, 2012, July 9, 2012, August 31, 2012, October 14, 2013 and November 14, 2013.

\section{A.15 Cyprus}

Decision 1 on Cyprus was taken by the Eurogroup in April 12, 2013 stating that "The Eurogroup has reached an agreement with the Cypriot authorities on the key elements necessary for a future macroeconomic adjustment program. [...] The Eurogroup welcomes the plans for restructuring the financial sector as specified in the annex. [...] financial assistance to Cyprus is warranted to safeguard financial stability in Cyprus and the euro area as a whole by providing financial assistance for an amount of up to EUR 10bn. [...] The Eurogroup expects that the ESM Board of Governors will be in a position to formally approve the proposal for a financial assistance facility agreement by the third week of April 2013 subject to the completion of national procedures." The first statement 1 regarding Cyprus corresponds to the Eurogroup meeting of March 4, 2013 where they declared that "the Eurogroup reiterates its readiness to assist Cyprus in its adjustment effort, including of its banking sector, in order to bring the economy to a sustainable growth path with sound public finances and to safeguard financial stability." The monitoring group 1 for Cyprus comprises the Eurogroup statements of June 27, 2012, December 13, 2012, March 16, 2013, March 18, 2013, March 19, 2013, March 21, 2013, March 24, 2013, May 13, 2013, September 12, 2013, September 13, 2013, February 17, 2014, March 10, 2014, June 19, 2014, October 13, 2014, November 6, 2014, February 16, 2015, June 18, 2015, September 12, 2015, January 14, 2016 and March 7, 2016.

\section{A.16 Two Pack}

Decision 1 on Two Pack was taken by the Council of the European Union in May 13, 2013 stating that "It adopted conclusions on: macroeconomic imbalances, in the light of in-depth reviews of imbalances in 13 member states; SEPA, the single euro payments area; the fast-start financing of climate change measures." The first statement 1 regarding Two Pack corresponds to the Council of the European Union meeting of October 19, 2012 where they declared that "The European Council invites the legislators to find an agreement with a view to adopting the two-pack by the end of 2012 at the latest." The monitoring group 
1 for Cyprus comprises the Council of the European Union statements of January 24, 2012 and November 13, 2012.

\section{A.17 SSM}

Decision 1 on SSM was taken by the Council of the European Union in October 15, 2013 stating that "The Council today adopted regulations creating a single supervisory mechanism for the oversight of banks and other credit institutions, thus establishing one of the main elements of Europe's banking union." The first statement 1 regarding SSM corresponds to the Eurogroup meeting of July 9, 2012 where they declared that "The Eurogroup welcomes the Commission's intention to present proposals in early September, notably on the basis of article 127(6) TFEU, for a single supervisory mechanism involving the ECB. We expect the Council to consider these proposals as a matter of urgency by the end of 2012." The monitoring group 1 for SSM comprises the European Council statement of October 19, 2012; and the Council of the European Union statements of November 13, 2012 and December 13, 2012 .

\section{A.18 Single Mortgage Credit}

Decision 1 on Single Mortgage Credit was taken by the Council of the European Union in January 28, 2014 stating that "The Council adopted a directive aimed at creating a single market for mortgage credits. This follows an agreement reached with the European Parliament at first reading."

\section{A.19 Deposit Guarantee Schemes}

Decision 1 on Deposit Guarantee Schemes was taken by the Council of the European Union in March 3, 2014 stating that "In order to make it easier to take up and pursue the business of credit institutions, it is necessary to eliminate certain differences between the laws of the Member States as regards the rules on deposit guarantee schemes (DGSs) to which those credit institutions are subject." The first statement 1 regarding Deposit Guarantee Schemes corresponds to the European Council meeting of October 23, 2011 where they declared that "Strengthening financial regulation remains a key priority at the EU and the global level. $[\ldots]$ calls for the speedy adoption of other important legislative proposals such as those relating to OTC derivatives and deposit guarantee schemes by the end of this year, and the ones on capital requirements by summer 2012." The monitoring group 1 for Deposit 
Guarantee Schemes comprises the Council of the European Union statement of February 18, 2014 .

\section{A.20 SRM}

Decision 1 on SRM was taken by the Council of the European Union in July 14, 2014 stating that "The Permanent Representatives Committee today approved, on behalf of the Council, a compromise agreed with the European Parliament on a regulation establishing a single resolution mechanism for failing banks. This enables the Parliament to approve the text at first reading [...]." The first statement 1 regarding SRM corresponds to the European Council meeting of October 19, 2012 where they declared that "The European Council notes the Commission's intention to propose a single resolution mechanism for Member States participating in the SSM once the proposals for a Recovery and Resolution Directive and for a Deposit Guarantee Scheme Directive have been adopted." The monitoring group 1 for SRM comprises the Council of the European Union statements of December 13, 2012, November 15, 2013; December 18, 2013; February 18, 2014; March 11, 2014; March 20, 2014; March 27, 2014 and December 19, 2014; and the European Council statements of October 25, 2013 and March 21, 2014.

\section{A.21 Bank Recovery and Resolution}

Decision 1 on Bank Recovery and Resolution was taken by the Council of the European Union in May 6, 2014 stating that "The Council today adopted a directive harmonizing national rules on bank recovery and resolution (PE-CONS 14/14)." The first statement 1 regarding Bank Recovery and Resolution corresponds to the Council of the European Union meeting of November 13, 2012 where they declared that "The two proposed regulations one conferring specific supervisory tasks on the European Central Bank, the other modifying regulation 1093/2010 establishing the European Banking Authority - are a key element of a broader plan to establish a banking union for the euro area. The plan also provides for a common resolution authority and a common deposit guarantee scheme." The monitoring group 1 for Bank Recovery and Resolution comprises the European Council statements of December 14, 2012, October 25, 2013 and December 11, 2013; and the Council of the European Union statements of May 14, 2013, May 21, 2014 and October 14, 2014. Decision 2 on Bank Recovery and Resolution was taken by the Council of the European Union in May 14, 2019 stating that "The Council today adopted a comprehensive legislative package 
which will reduce risks in the banking sector and further reinforce banks' ability to withstand potential shocks. The package contains amendments to the capital requirement legislation (regulation 575/2013 and directive 2013/36/EU) which reinforce the capital and liquidity positions of banks, and strengthens the framework for the recovery and resolution of banks in difficulty (directive 2014/59/EU and regulation 806/2014)." The first statement 2 regarding Bank Recovery and Resolution corresponds to the Council of the European Union meeting of December 5, 2017 where they declared that "Progress has been achieved on the banking package, and preliminary agreement has been reached on a broad range of issues. A number of political issues are still to be resolved, however, and these are identified in a progress report." The monitoring group 2 for Bank Recovery and Resolution comprises the Council of the European Union statements of January 23, 2018 and May 25, 2018.

\section{A.22 ELTIFs}

Decision 1 on ELTIFs was taken by the Council of the European Union in April 20, 2015 stating that "The Council adopted on April 20, 2015 a regulation aimed at increasing the pool of capital available for long-term investment in the EU economt by creating a new form of fund vehicle" The first statement regarding ELTIFs corresponds to the Council of the EU meeting of June 25, 2014 stating that " The Permanent Representatives Committee today agreed, on behalf of the Council, its position on a proposed regulation aimed at increasing the pool of capital available for long-term investment into the EU economy by creating a new form of fund vehicle".

\section{A.23 Rules on Capital shortfalls and Burdens}

Decision 1 on Rules on Capital shortfalls and Burdens was taken by the Council of the European Union in July 8, 2014 stating that "Building on the November 2013 ECOFIN statement, the current Terms of Reference aim at setting out a common understanding on process, principles on addressing capital shortfalls and applicable rules for burden sharing in the light of the outcome of the European Central Bank's comprehensive assessment and the EBA EU wide stress test, the results of which are to be published in the autumn of this year."

\section{A.24 Central Securities Depositories}

Decision 1 on Central Securities Depositories was taken by the Council of the European Union in July 23, 2014 stating that "The Council today adopted a regulation aimed at 
improving safety in the securities settlement system and opening the market for central securities depositories (CSD) services [...]." The first statement regarding Central Securities Depositories corresponds to the Council of the European Union meeting of February 26, 2014 where they declared that "The Permanent Representatives Committee today approved, on behalf of the Council, an agreement reached with the European Parliament on new rules aimed at improving safety in the securities settlement system and at opening the market for central securities depositories (CSD) services [...]."

\section{A.25 Transparency for Securities Financing Transac- tions}

Decision 1 on Transparency for Securities Financing Transactions was taken by the Council of the European Union in November 16, 2015 stating that "A regulation that will improve the transparency of securities lending and repurchase transactions was adopted by the Council on November 16, 2015". The first statement regarding Transparency for Securities Financing Transactions corresponds to the Council of the European Union meeting of November 10, 2014 where they declared that "The Council adopted a regulation aimed at improving market transparency for retail investors."

\section{A.26 SRF}

Decision 1 on SRF was taken by the Council of the European Union in November 30, 2015 stating that "Under the IGA, the fund will be built up over eight years, reaching a target level of at least $1 \%$ of the amount of covered deposits of all credit institutions authorized in all the participating member states. It is estimated that this will amount to about EUR 55bn." The first statement 1 regarding SRF corresponds to the Council of the European Union meeting of November 15, 2013 where they declared that "The Council discussed a draft regulation aimed at establishing a single decision-making authority and a single fund for the resolution of failing banks." The monitoring group 1 for SRF comprises the Council of the European Union statements of February 18, 2014, March 11, 2014, March 20, 2014, December 9, 2014, March 10, 2015 and June 19, 2015; the Eurogroup statements of February 17, 2014 and April 24, 2015; and the European Council statement of March 21, 2014. Decision 2 on SRF was taken by the Council of the European Union in December 8, 2015 stating that "The Single Resolution Board (SRB) welcomes the endorsement of the public bridge financing arrangement for the Single Resolution Fund (SRF) at today's meeting of the Economic and 
Financial Affairs Council (ECOFIN)." The first statement 2 regarding SRF corresponds to the Eurogroup meeting of July 13, 2015 where they declared that "Today we also looked at the issue of bridge financing, because of course there are urgent needs. And this process of finalizing the agreement will take time. This is very complex. We looked at a number of possibilities. There are technical, legal, financial and political issues to consider. So we have tasked an ad hoc working group, technical experts to look into that. They have already started today and they will continue their work tonight. Tomorrow, the Euro Working Group will look at it." The monitoring group 2 for SRF comprises the Council of the European Union statement of September 12, 2015. Decision 3 on SRF was taken by the Eurogroup in December 4, 2018 stating that "We agreed on the main details for the operationalization of the common backstop to the SRF [...]." The first statement 3 regarding SRF corresponds to the Council of the European Union meeting of December 18, 2013 where they declared that "Eurogroup and ECOFIN ministers also adopted a statement on the design of a backstop to the single resolution fund [...]." The monitoring group 3 for SRF comprises the Eurogroup statements of October 9, 2017, February 19, 2018, March 12, 2018, May 24, 2018, September 7, 2018, November 5, 2018 and November 19, 2018; and the Euro Summits of June 29, 2018 and December 14, 2018. Decision 4 on SRF was taken by the Eurogroup in December 4, 2019 stating that "[...] we have agreed on the ESM legal framework on the common backstop." The first statement 4 regarding SRF corresponds to the Eurogroup meeting of November 7 , 2019 where they declared that "We also took note of the good progress made on the common backstop, and more specifically on the Guideline and Board of Governors resolutions on the main features of the backstop. Views are converging on the nominal cap, i.e., the absolute limit to the amount that the ESM could lend to the SRF [...]." The monitoring group 4 for SRF comprises the Eurogroup statement of January 20, 2020.

\section{A.27 Insurance Distribution Rules}

Decision 1 on Insurance Distribution Rules was taken by the Council of the European Union in December 14, 2015 stating that "The Council adopted on 14 December 2015 a directive establishing new rules on insurance distribution, improving consumer protection for insurance products." The first statement 1 regarding Insurance Distribution Rules corresponds to the Council of the European Union meeting of November 5, 2014 where they declared that "The Permanent Representatives Committee agreed today, on behalf of the Council, its position on a draft directive establishing new improved rules on insurance mediation (14791/14)." 
The monitoring group 4 for SRF comprises the Council of the European Union statements of June 30, 2015, July 22, 2015, May 29, 2017, February 14, 2018, March 9, 2018 and March 20, 2018.

\section{A.28 Occupational Pension Funds}

Decision 1 on Occupational Pension Funds was taken by the Council of the European Union in December 8, 2016 stating that "On 8 December 2016, the Council adopted a directive on institutions for occupational retirement provision (IORPs), aimed at facilitating their development and better protecting pension scheme members and beneficiaries." The first statement 1 regarding Occupational Pension Funds corresponds to the Council of the European Union meeting of December 10, 2014 where they declared that "The Permanent Representatives Committee agreed, on behalf of the Council, its negotiating stance on a draft directive aimed at facilitating the development of occupational retirement savings and better protecting pension scheme members and beneficiaries (15901/2/14 REV 2)." The monitoring group 1 for Occupational Pension Funds comprises the Council of the European Union statement of June 30, 2016.

\section{A.29 Structural Reforms}

Decision 1 on Structural Reforms was taken by the Council of the European Union in May 11, 2017 stating that "On 11 May 2017, the Council approved a EUR 142.8 million program to help member states implement structural reforms." The first statement 1 regarding Structural Reforms corresponds to the Council of the European Union meeting of April 28, 2016 where they declared that "On 28 April 2016, the Permanent Representatives Committee (Coreper) agreed, on behalf of the Council, its negotiating stance on a proposed regulation establishing a structural reform program to help member states implement reforms." The monitoring group 1 for Structural Reforms comprises the Council of the European Union statement of February 8, 2017.

\section{A.30 New Prospectus Rules}

Decision 1 on New Prospectus Rules was taken by the Council of the European Union in May 16, 2017 stating that "On 16 May 2017, the Council adopted new rules on prospectuses for the issuing and offering of securities." The first statement 1 regarding New Prospectus Rules corresponds to the Council of the European Union meeting of June 8, 2016 where 
they declared that "On 8 June 2016, the Permanent Representatives Committee (Coreper) agreed, on behalf of the Council, a negotiating stance on new rules on prospectuses for the issuing and offering of securities." The monitoring group 1 for New Prospectus Rules comprises the Council of the European Union statements of June 17, 2016, December 8, 2016 and December 20, 2016.

\section{A.31 Money Market Funds}

Decision 1 on Money Market Funds was taken by the Council of the European Union in May 16, 2017 stating that "[.. ] the Council adopted a regulation to ensure the smooth operation of the short-term financing market." The first statement 1 regarding Money Market Funds corresponds to the Council of the European Union meeting of June 15, 2016 where they declared that "On 15 June 2016, the Permanent Representatives Committee (Coreper) agreed, on behalf of the Council, a negotiating stance on a draft regulation on money market funds, aimed at making such products more robust." The monitoring group 1 for Money Market Funds comprises the Council of the European Union statements of June 17, 2016, November 15, 2016 and December 7, 2016.

\section{A.32 Venture Capital Rules}

Decision 1 on Venture Capital Rules was taken by the Council of the European Union in October 9, 2017 stating that "On 9 October 2017, the Council adopted new venture capital rules aimed at boosting investment in start-ups and innovation." The first statement 1 regarding Venture Capital Rules corresponds to the Council of the European Union meeting of December 16, 2016 where they declared that "The Council has agreed its negotiating stance on amendments to EU rules aimed at boosting investment in venture capital and social enterprises."

\section{A.33 Securitization Rules}

Decision 1 on Securitization Rules was taken by the Council of the European Union in November 20, 2017 stating that "On 20 November 2017, the Council adopted rules aimed at facilitating the development of a securitization market in Europe." The first statement 1 regarding Securitization Rules corresponds to the Council of the European Union meeting of December 2, 2015 where they declared that "On 2 December 2015, the Permanent Representatives Committee (Coreper) approved, on behalf of the Council, a negotiating stance on 
proposals aimed at facilitating the development of a securitization market in Europe." The monitoring group 1 for Securitization Rules comprises the Council of the European Union statements of May 30, 2017 and July 13, 2018.

\section{A.34 Procedures to reduce banking risk}

Decision 1 on Risk Reduction was taken by the Council of the European Union in December 7, 2017 stating that "These two legislative acts were fast-tracked from amongst a series of measures aimed at reducing risk in the banking industry", said Toomas Tõniste, minister for finance of Estonia, which currently holds the Council presidency." The first statement 1 regarding Risk Reduction corresponds to the Council of the European Union meeting of June 16, 2017 where they declared that "The Council agreed its stance on part of a package of proposals aimed at reducing risk in the banking sector, $[\ldots]$." The monitoring group 1 for Risk Reduction comprises the Council of the European Union statement of November 15, 2017, December 4, 2018 and February 15, 2019; and the Eurogroup statement of November 19, 2018. Decision 2 on Risk Reduction was taken by the Council of the European Union in May 14, 2019 stating that "The EU will soon have in place simplified rules for non-financial counterparties, small financial counterparties and pension funds using financial derivative products."

\section{A.35 Payment Services}

Decision 1 on Payment Services was taken by the Council of the European Union in March 4, 2019 stating that "The Council today adopted a regulation on aligning the costs of crossborder payments in euros between euro and non-euro countries and increasing the transparency of charges related to currency conversion services across the EU." The first statement 1 regarding payment services corresponds to the Council of the EU in May 5, 2015 stating that "The Latvian presidency of the Council and the European Parliament on 5 May 2015 reached a tentative agreement on a draft directive aimed at further developing an EU-wide market for electronic payments." The monitoring group 1 for payment services comprises the Council of the EU meetings of June 4, 2015 and December 19, 2018.

\section{A.36 NPLs}

Decision 1 on NPLs was taken by the Council of the European Union in April 9, 2019 stating that "The Council today adopted a new framework for dealing with banks' bad loans. The 
new rules set capital requirements applying to banks with non-performing loans (NPLs) on their balance sheets." The first statement 1 regarding NPLs corresponds to the Council of the European Union meeting of June 16, 2017 where they declared that "Actions are envisaged in the following areas: [...] structural reforms to enable the banking industry better manage the timely resolution of NPLs." The monitoring group 1 for NPLs comprises the Council of the European Union statements of June 16, 2017, July 11, 2017, December 5, 2017, January 23, 2018, October 31, 2018, December 4, 2018, December 18, 2018 and March 27, 2019; the Eurogroup statements of November 7, 2016, July 10, 2017, November 5, 2018 and November 19, 2018; and the Euro Summit of December 14, 2018.

\section{A.37 Financial Derivative Products and Cleaning}

Decision 1 on Financial Derivative Products and Cleaning was taken by the Council of the European Union in May 142019 stating that "The Council today adopted a regulation improving the existing regulatory framework applying to the over-the-counter (OTC) derivative market." The first statement 1 regarding Financial Derivative Products and Cleaning corresponds to the Council of the European Union meeting of February 5, 2019 where they declared that "The Romanian presidency of the Council and the European Parliament reached today a preliminary agreement on improving the existing regulatory framework applying to the over-the-counter (OTC) derivative market."

\section{A.38 BICC}

Decision 1 on BICC was taken by the Council of the European Union in June 13, 2019 stating that "[...] we agreed on the main features of the budgetary instrument for convergence and competitiveness (BICC) for the euro area, [... ]." The first statement 1 regarding BICC corresponds to the Council of the European Union meeting of May 14, 2013 where they declared that "The Council took note of the presentation by the Commission of two communications on the further development of the EU's economic and monetary union (EMU). It held a brief exchange of views. The communications relate to: introduction of a "convergence and competitiveness instrument" (6849/13); [...]." The monitoring group 1 for BICC comprises the Eurogroup statements of November 19, 2018, December 4, 2018, January 21, 2019; February 11, 2019; March 19, 2019; April 5, 2019; May 16, 2019; September 13, 2019; November 7, 2019; December 4, 2019; January 20, 2020 and February 17, 2020; and the Euro Summits of December 14, 2018 and June 21, 2019. Decision 2 on BICC was taken by the Eurogroup in 
October 9, 2019 stating that "[...] ministers agreed on all the critical elements of governance and financing, which make BICC an innovative tool, different from other EU funds[...]."

\section{A.39 Pension Products}

Decision 1 on Pension Products was taken by the Council of the European Union in June 14, 2019 stating that "The Council today adopted two key reforms in the framework of the Capital Markets Union. They adopted: a regulation providing greater choice for people who wish to save for their retirement and expanding the market for personal pensions through the creation of a 'pan-European pension product' (PEPPs)." The first statement 1 regarding Pension Products corresponds to the Council of the European Union meeting of February 13, 2019 where they declared that "EU institutions agreed new rules that will make it easier for people to put money aside for their retirement. EU ambassadors today endorsed the agreement reached between the presidency and the European Parliament on 13 December on the proposed 'pan-European pension product' (PEPP), a new class of personal pension scheme."

\section{A.40 Cross Border Investment Funds}

Decision 1 on Cross Border Investment Funds was taken by the Council of the European Union in June 14, 2019 stating that "The Council today adopted two key reforms in the framework of the Capital Markets Union. They adopted: [...] a package of measures aimed at removing existing barriers to the cross-border distribution of investment funds." The first statement 1 regarding Cross Border Investment Funds corresponds to the Council of the European Union meeting of February 5, 2019 where they declared that "The Romanian presidency of the Council and the European Parliament reached today a preliminary agreement on a package of measures aimed at removing existing barriers to the cross-border distribution of investment funds. The deal will now be submitted for endorsement by EU ambassadors."

\section{A.41 Clearing House Rules}

Decision 1 on Clearing House Rules was taken by the Council of the European Union in October 15, 2019 stating that "The new framework sets out how EU and third country clearing houses should be supervised in the future, taking particular account of the effects of Brexit on the European financial system." The first statement 1 regarding Clearing House Rules 
corresponds to the Council of the European Union meeting of March 13, 2019 where they declared that "The Presidency and the Parliament reached today a provisional agreement on how EU and third country clearing houses should be supervised in the future, taking into particular account the effects of Brexit on the European financial system."

\section{A.42 Set of CMU legislative reforms}

Decision 1 on a set of CMU legislative reforms was taken by the Council of the EU in November 8, 2019 stating that "The council adopted a set of legislative reforms which are part of progress towards the capital markets union. The text concerns the creation of a new category of benchmarks contributing to sustainable finance, transparency obligations for sustainable investments, a new prudential framework for investment firms, a harmonised framework for covered bonds and rules promoting access to SME growth markets." The monitoring group for the set of CMU legislative reforms comprises the Council of the EU meetings of November 28, 2018; January 7, 2019; February 25, 2019; February 27, 2019; March 7, 2019 and March 20, 2019.

\section{A.43 General Escape Clause of the EU Fiscal Frame- work}

Decision 1 on General Escape Clause of the EU Fiscal Framework was taken by the Council of the European Union in March 23, 2020 stating that " [.. ] taking into account the significant negative impact that COVID-19 virus has on the stability of Member States' public finances, "the ministers agreed that the conditions for the use of general escape clause of the EU fiscal framework are fulfilled. This will give Member States the opportunity to go further with providing fiscal incentives to their economies" said deputy prime minister and minister of finance Zdravko Marić. The first statement 1 regarding General Escape Clause of the EU Fiscal Framework corresponds to the Eurogroup meeting of March 4, 2020 where they declared that "We are prepared to take further policy action. This includes fiscal measures - where appropriate, as they may be needed to support growth. Our fiscal rules framework provides for flexibility to cater for "unusual events outside the control of government". It is up for the European Commission to implement these rules and assess requests from member states. To be specific, in the rules of the Stability and Growth Pact, this clause allows for a temporary deviation from the adjustment path, while preserving fiscal sustainability." The monitoring group 1 for General Escape Clause of the EU Fiscal Framework comprises the European Council statements of March 10, 2020, March 17, 2020 and March 26, 2020. 
Table A.1: The narrative

\begin{tabular}{|c|c|c|c|c|c|}
\hline \multicolumn{2}{|c|}{ One Country } & \multicolumn{3}{|c|}{ Whole area } & $\frac{\text { None }}{\text { Topics not included }}$ \\
\hline \multirow{2}{*}{ Greek 1st bailout } & deficit & & Depositories & & on exit strategies of \\
\hline & & & & & the EERP \\
\hline \multirow{3}{*}{ Irish bailout } & & financial system: & & & investment funds \\
\hline & & ESMA, ESRB, EBA & & & \\
\hline & & and EIPA & & & \\
\hline \multirow[t]{2}{*}{ Portuguese bailout } & EDP Denmark & EFSM & Transparency rules & Euro Plus Pact & Task Force \\
\hline & & & transaction & & \\
\hline \multirow{3}{*}{$\begin{array}{l}\text { Greek debt } \\
\text { restructuration }\end{array}$} & Excessive deficit & EFSF & Insurance & Two-pack & Harmonisation \\
\hline & Cyprus & & Distribution rules & & short-selling and \\
\hline & & & & & credit default swaps \\
\hline \multirow[t]{2}{*}{ Greek 2nd bailout } & EDP Finland & Bank capital & Occupational & Fiscal Compact & Tax Policy \\
\hline & & requirements & pension funds & & coordination \\
\hline
\end{tabular}


Table A.1: (continued)

\begin{tabular}{|c|c|c|c|c|c|}
\hline \multicolumn{2}{|c|}{ One Country } & \multicolumn{4}{|c|}{ Whole area } \\
\hline Bailout & Deficit & Financial & $C M U$ & Fiscal & None \\
\hline \multirow[t]{2}{*}{ Spanish bailout } & Irish excessive & National deposit & New prospectus & $\mathrm{BICC}$ & SGP into national \\
\hline & deficit & guarantee schemes & rules & & legislation \\
\hline \multirow[t]{3}{*}{ Cypriot bailout } & Portuguese & ESM & Money market funds & - & Report "Towards a \\
\hline & excessive deficit & & & & stronger Economic \\
\hline & & & & & Union" \\
\hline \multirow{3}{*}{$\begin{array}{c}\text { Extension maturity } \\
\text { Ireland and } \\
\text { Portugal }\end{array}$} & Spanish excessive & OTC & Venture capital & - & Eurobonds \\
\hline & deficit & & rules & & \\
\hline & & & & & \\
\hline \multirow[t]{2}{*}{ Greek 3rd bailout } & Italian excessive & Bank recovery and & Securitisation rules & - & $4 \mathrm{PR}$ \\
\hline & deficit & resolution & & & \\
\hline \multirow{4}{*}{$\begin{array}{c}\text { Greek } \\
\text { policy-contingent } \\
\text { debt measures }\end{array}$} & EDP Germany & SSM & Reducing charges & - & Multiannual \\
\hline & & & and increasing & & Financial \\
\hline & & & transparency in & & Framework \\
\hline & & & payment services & & \\
\hline
\end{tabular}


Table A.1: (continued)

\begin{tabular}{|c|c|c|c|c|c|}
\hline \multicolumn{2}{|c|}{ One Country } & \multicolumn{4}{|c|}{ Whole area } \\
\hline \multirow[t]{3}{*}{ Bailout } & Deficit & Financial & $C M U$ & Fiscal & None \\
\hline & \multirow{2}{*}{ EDP Latvia } & \multirow{2}{*}{$\mathrm{SRF}$} & financial derivatives & \multirow{2}{*}{-} & \multirow{2}{*}{ EU budgets } \\
\hline & & & products and & & \\
\hline & EDP Lithuania & SRM & Pension products & - & Insurance mediation \\
\hline \multirow{3}{*}{\multicolumn{2}{|c|}{ EDP Hungary }} & \multirow[t]{3}{*}{ SRB } & Cross-border & \multirow[t]{3}{*}{-} & Transparency rules \\
\hline & & & investment funds & & for retail investment \\
\hline & & & & & products \\
\hline \multirow{2}{*}{\multicolumn{2}{|c|}{ EDP France }} & Bank resolution & New benchmarks for & - & EFSI \\
\hline & & fund & sustainable finance & & \\
\hline & \multirow[t]{3}{*}{ EDP Poland } & Rules on capital & New prudential & \multirow[t]{3}{*}{-} & \multirow[t]{3}{*}{$5 \mathrm{PR}$} \\
\hline & & shortfalls and & framework for & & \\
\hline & & burden sharing & financial institutions & & \\
\hline
\end{tabular}


Table A.1: (continued)

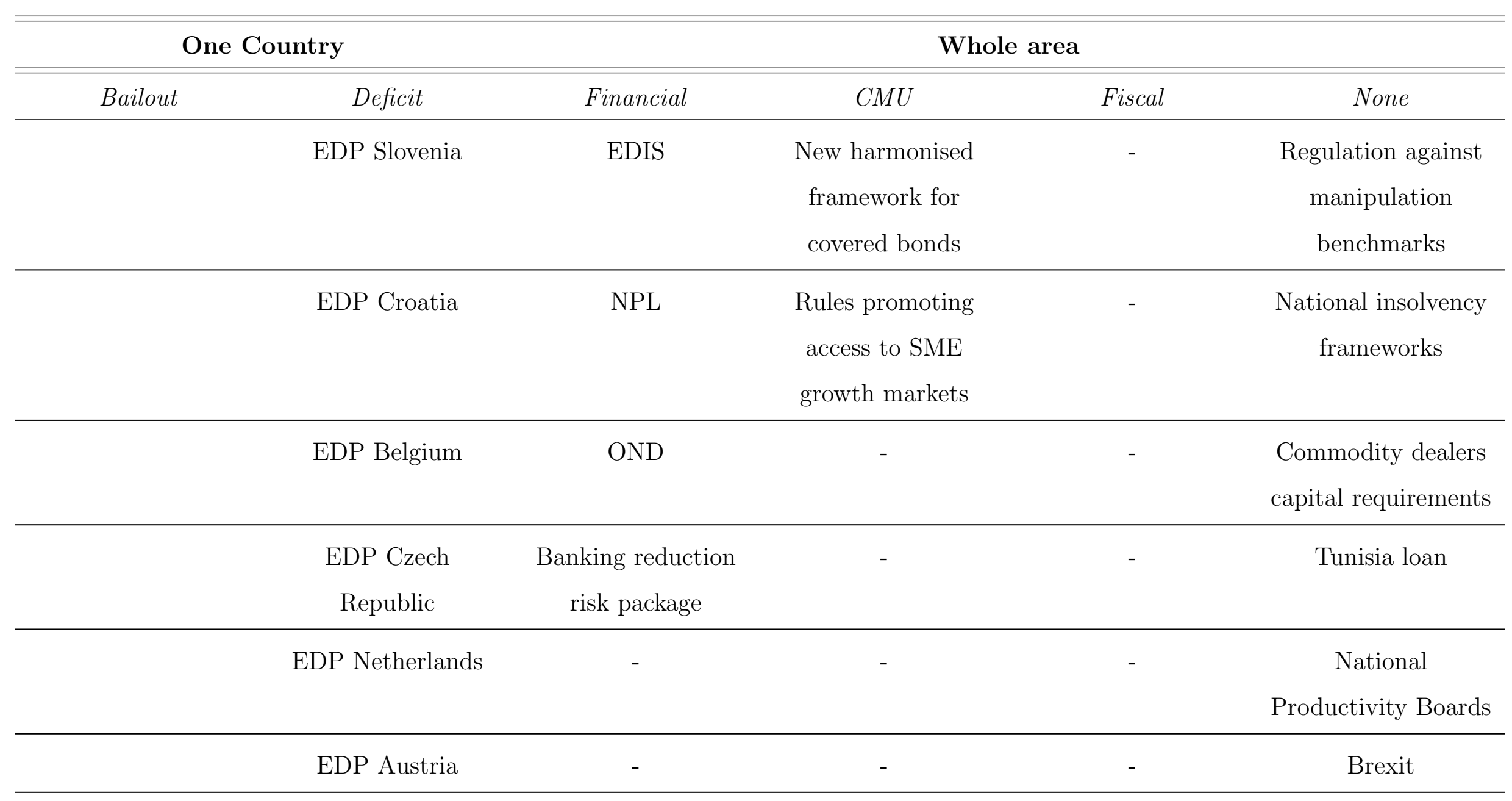


Table A.1: (continued)

\begin{tabular}{|c|c|c|c|c|c|}
\hline \multicolumn{2}{|c|}{ One Country } & \multicolumn{4}{|c|}{ Whole area } \\
\hline Bailout & Deficit & Financial & $C M U$ & Fiscal & None \\
\hline \multirow{4}{*}{\multicolumn{2}{|c|}{ EDP Slovakia }} & - & - & - & Consumer \\
\hline & & & & & involvement in \\
\hline & & & & & financial service \\
\hline & & & & & policy-making \\
\hline \multicolumn{2}{|r|}{ EDP Malta } & - & - & - & Moldova loan \\
\hline \multirow{2}{*}{\multicolumn{2}{|c|}{$\begin{array}{c}\text { Spain and Portugal } \\
\text { fines }\end{array}$}} & - & - & - & Insurance agreement \\
\hline & & & & & with US \\
\hline \multirow{2}{*}{\multicolumn{2}{|c|}{$\begin{array}{l}\text { EDP United } \\
\text { Kingdom }\end{array}$}} & - & - & - & Regulation against \\
\hline & & & & & tax avoidance \\
\hline \multicolumn{2}{|r|}{$\begin{array}{c}\text { Escape clause fiscal } \\
\text { framework }\end{array}$} & - & - & - & $\mathrm{EMF}$ \\
\hline \multirow{2}{*}{\multicolumn{2}{|c|}{$\begin{array}{c}\text { Structural reform } \\
\text { program }\end{array}$}} & - & - & - & Georgia loan \\
\hline & & & & & \\
\hline & - & - & - & - & Omnibus regulation \\
\hline & - & - & - & - & Invest EU \\
\hline
\end{tabular}


Table A.1: (continued)

\begin{tabular}{|c|c|c|c|c|c|}
\hline \multicolumn{2}{|c|}{ One Country } & \multicolumn{4}{|c|}{ Whole area } \\
\hline \multirow[t]{5}{*}{ Bailout } & Deficit & Financial & $C M U$ & Fiscal & None \\
\hline & - & - & - & - & Macroeconomic \\
\hline & & & & & dialogues \\
\hline & - & - & - & - & Crowfunding \\
\hline & & & & & platforms regulation \\
\hline
\end{tabular}

Notes: The last column highlights some of the not included topics, either because they did not end up with a final decision or because we consider the decision is not relevant. Regarding the latter, the benchmark we use to discard the decisions is that they do not fit in any of the three categories we propose (fiscal, crisis management or integration) or because they are not a decision per se (for instance, the 5 Presidents Report). 


\section{Annex B: Detailed description of our decisions}

Table B.1: Fiscal decisions

\begin{aligned} & \multicolumn{1}{l}{ Date } Main decisions \\ & \hline $7 / 13 / 10$ EDP Bulgaria, Denmark, Cyprus and Finland opened \\ & \hline $11 / 16 / 10$ Greek deficit \\ & \hline $7 / 12 / 11$ EDP Finland closed \\ & \hline $6 / 22 / 12$ EDP Germany and Bulgaria closed \\ & \hline $10 / 9 / 12$ Portugal 1 year more to correct deficit \\ & \hline $6 / 21 / 13$ EDP Italy, Latvia, Lithuania, Hungary and Romania closed \\ & \hline $6 / 21 / 13$ Deadlines for Spain, France, Poland and Slovenia, Netherlands and Portugal for \\ & \hline $1 / 28 / 14$ EDP Croatia opened \\ & \hline $6 / 20 / 14$ EDP Belgium, Czech Republic, Denmark, Netherlands, Austria and Slovakia closed \\ & \hline $3 / 10 / 15$ France has 2 more years to reduce deficit \\ & \hline $6 / 19 / 15$ EDP Malta and Poland closed \\ & \hline $7 / 12 / 15$ Spain fined for misreporting \\ & \hline $6 / 17 / 16$ EDP Cyprus, Ireland and Slovenia closed \\ & \hline $7 / 12 / 16$ Fines approved for not meeting deficit objective Portugal and Spain \\ & \hline $8 / 9 / 16$ Agreement to cancel fines to Spain and Portugal and extend deadline \\ & \hline $5 / 11 / 17$ Structural reform support programme \\ & \hline $6 / 16 / 17$ EDP Croatia and Portugal closed \\ & \hline $6 / 25 / 17$ EDP Greece closed \\ & \hline $12 / 5 / 17$ EDP United Kingdom closed \\ & \hline $6 / 22 / 18$ EDP France closed \\ & \hline $3 / 23 / 20$ Escape clause EU fiscal framework \\ & \hline \\ & \hline $6 / 19$ EDP Spain closed \\ & \hline 6\end{aligned}


Table B.2: Bailout decisions

\begin{aligned} & \multicolumn{1}{c}{ Date } Main decisions \\ & \hline $5 / 2 / 10$ Bailout Greece \\ & \hline $11 / 28 / 10$ Bailout Ireland \\ & \hline $5 / 16 / 11$ Bailout Portugal \\ & \hline $10 / 26 / 11$ Greek debt removal \\ & \hline $2 / 21 / 12$ Succesor program Greece \\ & \hline $7 / 20 / 12$ Bailout Spain \\ & \hline $11 / 27 / 12$ Updated program conditionality Greece \\ & \hline $4 / 12 / 13$ Agreement to lengthen the maturity of loans Portugal and Ireland and bailout Cyprus \\ & \hline $6 / 12 / 14$ Portugal foregoes last tranche of bailout \\ & \hline $6 / 27 / 15$ Rejection 3rd program Greece \\ & \hline $8 / 14 / 15$ Agreement on the policy conditionality of the ESM programme Greece \\ & \hline $6 / 21 / 18$ Greece successful program \\ & \hline $4 / 5 / 19$ Policy-contingent program Greece \\ & \hline\end{aligned}

Table B.3: CMU decisions

\begin{aligned} & \multicolumn{1}{l}{ Date } Main decisions \\ & \hline $7 / 23 / 14$ Central securities depositories \\ & \hline $4 / 20 / 15$ ELTIF \\ & \hline $11 / 16 / 15$ Transparency rules on securities financing transaction \\ & \hline $12 / 14 / 15$ Insurance distribution rules \\ & \hline $12 / 8 / 16$ Occupational pension funds \\ & \hline $5 / 16 / 17$ New prospectus rules \\ & \hline $5 / 16 / 17$ Money market funds \\ & \hline $10 / 9 / 17$ Venture capital rules \\ & \hline $3 / 4 / 19 / 17$ Securitisation rules \\ & \hline $5 / 14 / 19$ Reducing charges and increasing transparency in payment services \\ & \hline $6 / 14 / 19$ Pension products \\ & \hline $6 / 14 / 19$ Cross border investment funds \\ & \hline $10 / 15 / 19$ Clearing house rules \\ & \hline $11 / 8 / 19$ Set of CMU legislative reforms \\ & \hline\end{aligned}




\section{Annex C: Additional material and Robustness checks}

Table C.1: Robustness exercise. 1-day window for the event analysis. 10-year spread with respect to the OIS risk-free reference.

\begin{tabular}{|c|c|c|c|c|c|c|c|c|c|c|c|}
\hline VARIABLES & (1) & (2) & (3) & (4) & (5) & (6) & (7) & (8) & (9) & (10) & (11) \\
\hline Decision t-1 & -2.002 & -1.605 & -0.862 & -1.470 & -1.174 & -0.119 & -0.433 & -2.100 & -3.106 & -1.886 & -0.329 \\
\hline & $(1.671)$ & $\begin{array}{l}(1.634) \\
1.861\end{array}$ & $\begin{array}{l}(1.151) \\
2493 * *\end{array}$ & $(1.680)$ & $(1.296)$ & $(1.324)$ & $(1.228)$ & $(1.585)$ & $(2.387)$ & $(1.811)$ & $(1.436)$ \\
\hline Decision $t$ & $\begin{array}{l}-1.965 \\
(1.668)\end{array}$ & $\begin{array}{c}1.861 \\
(1.631)\end{array}$ & $\begin{array}{l}2.493^{* *} \\
(1.150)\end{array}$ & $\begin{array}{c}1.689 \\
(1.654)\end{array}$ & $\begin{array}{c}1.478 \\
(1.271)\end{array}$ & $\begin{array}{c}1.402 \\
(1.308)\end{array}$ & $\begin{array}{c}1.467 \\
(1.207)\end{array}$ & $\begin{array}{l}3.276^{* *} \\
(1.558)\end{array}$ & $\begin{array}{c}0.899 \\
(2.353)\end{array}$ & $\begin{array}{c}1.916 \\
(1.779)\end{array}$ & $\begin{array}{c}1.817 \\
(1.419)\end{array}$ \\
\hline Decision $t+1$ & $-4.096 * *$ & $-4.432^{* * *}$ & $-3.105^{* * *}$ & $-4.431^{* * *}$ & $-2.257^{*}$ & $\begin{array}{l}(1.5006 \\
-1.306\end{array}$ & $\begin{array}{l}-1.772 \\
-1.201\end{array}$ & $-5.481^{* * *}$ & $-6.483^{* * *}$ & $-3.113^{*}$ & $\begin{array}{l}1.419 * \\
-2.908^{* *}\end{array}$ \\
\hline & $(1.666)$ & $(1.617)$ & $(1.139)$ & $(1.638)$ & $(1.261)$ & $(1.292)$ & $(1.197)$ & $(1.546)$ & $(2.326)$ & $(1.782)$ & $(1.407)$ \\
\hline BIDASK & $\begin{array}{l}-0.342 \\
(2.143)\end{array}$ & $-2.870^{* * * *}$ & 0.689 & $-2.929^{* * * *}$ & $\begin{array}{l}4.296 \\
8.46\end{array}$ & 0.905 & 2.207 & -0.518 & $-3.524^{* * *}$ & $-3.067 * * *$ & $-2.983^{* * * *}$ \\
\hline & $\begin{array}{c}(2.143) \\
-2.693^{* * *}\end{array}$ & $\begin{array}{l}(0.660) \\
-0.791\end{array}$ & $\begin{array}{l}(1.024) \\
0.681\end{array}$ & $\begin{array}{l}(0.679) \\
-1.470\end{array}$ & $\begin{array}{l}(8.486) \\
-1.944\end{array}$ & $\begin{array}{l}(14.77) \\
-2.931\end{array}$ & $(8.826)$ & $(1.106)$ & (0.696) & $(0.668)$ & $(0.667)$ \\
\hline$\triangle \mathrm{EONIA}$ & $\begin{array}{c}-2.6933 \\
(0.670)\end{array}$ & $\begin{array}{l}-0.791 \\
(2.884)\end{array}$ & $\begin{array}{l}0.681 \\
(2.034)\end{array}$ & $\begin{array}{l}-1.470 \\
(2.912)\end{array}$ & $\begin{array}{l}-1.944 \\
(2.293)\end{array}$ & $\begin{array}{l}-2.931 \\
(2.353)\end{array}$ & $\begin{array}{l}-2.527 \\
(2.177)\end{array}$ & $\begin{array}{l}3.874 \\
(2.773)\end{array}$ & $\begin{array}{l}-2.016 \\
(4.106)\end{array}$ & $\begin{array}{c}0.137 \\
(2.898)\end{array}$ & $\begin{array}{l}-1.465 \\
(2.908)\end{array}$ \\
\hline VIX & -0.974 & $0.181^{* * *}$ & 0.0112 & $0.197^{* * *}$ & -0.0383 & -0.0357 & -0.0279 & -0.0164 & $0.312^{* * *}$ & $0.203^{* * * *}$ & $0.193^{* * *}$ \\
\hline & $(2.971)$ & $(0.0419)$ & $(0.0301)$ & $(0.0436)$ & $(0.0309)$ & $(0.0308)$ & $(0.0291)$ & $(0.0394)$ & $(0.0636)$ & $(0.0435)$ & $(0.0435)$ \\
\hline $\mathrm{ECB}$ & $\begin{array}{l}0.219 * * * \\
(0.0357)\end{array}$ & $\begin{array}{l}0.251 \\
(1.045)\end{array}$ & $\begin{array}{l}-1.077 \\
(0.732)\end{array}$ & $\begin{array}{c}0.190 \\
(1.061)\end{array}$ & $\begin{array}{c}-1.372^{*} \\
(0.815)\end{array}$ & $\begin{array}{c}-2.145 * * * \\
(0.832)\end{array}$ & $\begin{array}{c}-1.811^{* * *} \\
(0.773)\end{array}$ & $\begin{array}{l}-0.181 \\
(1.008)\end{array}$ & $\begin{array}{r}1.898 \\
(1.511)\end{array}$ & 0.213 & 0.172 \\
\hline$\triangle$ STOCK & 1.329 & $-0.0208^{* * *}$ & $-0.0164^{* * *}$ & $-0.0205^{* * *}$ & $-0.0256^{* * *}$ & $\begin{array}{r}(.832) * \\
-0.0129 * * *\end{array}$ & $-0.0148^{* * *}$ & $\begin{array}{r}(1.0085 * * * \\
-0.0655^{* *}\end{array}$ & $-0.0720^{* * *}$ & $\begin{array}{r}(1.052) * * \\
-0.0214^{* * *}\end{array}$ & $\begin{aligned} &(1.0600) \\
&-0.0212^{* * *}\end{aligned}$ \\
\hline & $(1.001)$ & $(0.000834)$ & $(0.000570)$ & $(0.000841)$ & $(0.00122)$ & $(0.000555)$ & $(0.000610)$ & $(0.00318)$ & $(0.00448)$ & $(0.000826)$ & $(0.000828)$ \\
\hline MOODY & $-0.0235 * * *$ & -1.153 & -2.786 & -3.415 & 1.041 & -1.472 & -0.000385 & -3.374 & -4.814 & -3.559 & -3.535 \\
\hline & $(0.000825)$ & (3.116) & (1.811) & (3.101) & $(2.788)$ & (3.771) & $(2.406)$ & $(2.564)$ & (4.103) & $(3.036)$ & $(3.048)$ \\
\hline DEFICIT & -0.914 & 1.539 & 0.631 & 1.827 & 1.785 & 2.037 & 2.046 & -1.875 & 1.104 & 1.425 & 1.511 \\
\hline & (3.166) & $(2.229)$ & $(1.581)$ & $(2.256)$ & $(1.754)$ & $(1.792)$ & (1.663) & $(2.147)$ & $(3.212)$ & $(2.231)$ & $(2.250)$ \\
\hline BAILOUT & & $\begin{array}{l}-3.243 \\
(3.073\end{array}$ & -2.396 & -3.434 & -0.934 & 0.403 & 0.0504 & $-6.287^{* *}$ & -6.764 & -3.490 & -3.430 \\
\hline & & $\begin{array}{c}(3.073) \\
-38 * * *\end{array}$ & $\begin{array}{c}(2.116) \\
(2.65 * * *\end{array}$ & $\begin{array}{l}(3.108) \\
-3.02 * * *\end{array}$ & $\begin{array}{c}(2.329) \\
\end{array}$ & $(2.381)$ & $(2.209)$ & $(3.029)$ & $\begin{array}{l}(4.593) \\
21\end{array}$ & $(3.084)$ & $(3.106)$ \\
\hline WIT & & $\begin{array}{c}-38.48^{* * *} \\
(10.38)\end{array}$ & $\begin{array}{c}-26.85^{* * *} * \\
(6.645)\end{array}$ & $\begin{array}{c}-37.82^{* * *} \\
(10.48)\end{array}$ & $\begin{array}{c}-49.79^{* * *} \\
(7.386)\end{array}$ & $\begin{array}{c}-37.01^{* * *} \\
(7.516)\end{array}$ & $\begin{array}{c}-44.76^{* * *} \\
(6.988)\end{array}$ & $\begin{array}{l}4.199 \\
(10.97)\end{array}$ & $\begin{array}{l}-31.87^{*} \\
(17.38)\end{array}$ & $\begin{array}{c}-37.50^{* * *} \\
(10.40)\end{array}$ & $\begin{array}{c}-37.63^{* * *} \\
(10.47)\end{array}$ \\
\hline WIT period & & $-1.891 * * *$ & $-1.426^{* * * *}$ & $-1.799 * * *$ & $-1.082^{* *}$ & $-0.862^{*}$ & $-0.973^{* *}$ & $-1.658^{* *}$ & $-2.523^{* *}$ & $-1.892^{* * *}$ & $-1.816^{* * * *}$ \\
\hline & & $(0.640)$ & $(0.459)$ & $\begin{array}{l}(0.668) \\
-8732\end{array}$ & $(0.473)$ & $(0.501)$ & $(0.448)$ & $(0.658)$ & $(1.012)$ & $(0.665)$ & $(0.667)$ \\
\hline APP & & $\begin{array}{l}-9.614 \\
(9.359)\end{array}$ & $\begin{array}{l}5.254 \\
(6.633)\end{array}$ & $\begin{array}{l}-8.732 \\
(9.470)\end{array}$ & $\begin{array}{l}4.147 \\
(.370)\end{array}$ & $\begin{array}{l}7.163 \\
(7.525)\end{array}$ & $\begin{array}{l}5.297 \\
(.990)\end{array}$ & $\begin{array}{l}4.775 \\
(8.846)\end{array}$ & $\begin{array}{l}-17.69 \\
(13.21)\end{array}$ & $\begin{array}{l}-8.598 \\
(9.392)\end{array}$ & $\begin{array}{l}-8.625 \\
(9.463)\end{array}$ \\
\hline APP period & & $-1.285^{* *}$ & $-0.883^{* *}$ & $-1.233^{* *}$ & $-0.839^{*}$ & -0.645 & $-0.723^{*}$ & $-1.260^{* * *}$ & $\begin{array}{l}\left(1.808^{* *}\right. \\
-1\end{array}$ & $\begin{aligned} &(.3932) \\
&-1.313^{* *}\end{aligned}$ & $\begin{array}{l}(9.463) \\
-1.219^{* *}\end{array}$ \\
\hline & & $(0.568)$ & $(0.412)$ & $(0.592)$ & $(0.446)$ & $(0.460)$ & $(0.415)$ & $(0.615)$ & $(0.899)$ & $(0.590)$ & $\begin{array}{c}-1.219 \text { * } \\
(0.590)\end{array}$ \\
\hline Two pack & & -12.65 & -4.316 & $-18.15^{*}$ & -7.568 & 8.724 & 0.802 & -8.569 & $-47.35^{* * *}$ & $-19.46^{*}$ & $-18.07^{*}$ \\
\hline & & (10.37) & $(6.644)$ & (10.47) & $(7.353)$ & $(7.502)$ & $(6.972)$ & $(10.96)$ & $(17.39)$ & $(10.40)$ & $(10.46)$ \\
\hline SSM & & -16.84 & $-21.91 * * *$ & -16.12 & $-37.26^{* * *}$ & $-19.78^{* * *}$ & $-29.71^{* * *}$ & -8.442 & -0.294 & -15.85 & -15.86 \\
\hline & & (10.39) & (6.658) & $(10.50)$ & $(7.380)$ & $(7.544)$ & (6.995) & (10.98) & (17.45) & (10.43) & (10.49) \\
\hline BICC & & -8.305 & -8.158 & -9.413 & -8.356 & $-16.11^{* *}$ & -11.24 & -5.852 & -7.891 & -9.459 & -9.350 \\
\hline & & (9.311) & $(6.600)$ & $(9.422)$ & $(7.340)$ & $(7.532)$ & (6.969) & (8.796) & (13.14) & $(9.344)$ & $(9.415)$ \\
\hline SRM & & 9.715 & 2.506 & 9.718 & 9.279 & 6.407 & 7.944 & -1.043 & 10.72 & 9.729 & 9.730 \\
\hline & & $(9.314)$ & (6.601) & $(9.423)$ & $(7.341)$ & $(7.489)$ & $(6.961)$ & $(8.799)$ & (13.14) & $(9.345)$ & $(9.416)$ \\
\hline EDIS & & $\begin{array}{l}-0.572 \\
(9.311\end{array}$ & $\begin{array}{l}-4.829 \\
6.599\end{array}$ & $\begin{array}{l}-0.516 \\
-(9.416\end{array}$ & $\begin{array}{l}-4.965 \\
(7.333\end{array}$ & $\begin{array}{l}-7.861 \\
(7.486\end{array}$ & $\begin{array}{l}-6.446 \\
-6956\end{array}$ & $\begin{array}{l}-3.351 \\
8.794\end{array}$ & $\begin{array}{l}4.405 \\
(13.13\end{array}$ & $\begin{array}{l}-0.561 \\
(9.343\end{array}$ & $\begin{array}{l}-0.593 \\
(9.414\end{array}$ \\
\hline & & (9.311) & $(6.599)$ & $\begin{array}{l}(9.421) \\
1230\end{array}$ & $\begin{array}{l}(7.333) \\
1.905\end{array}$ & $(7.486)$ & $(6.956)$ & $\begin{array}{l}(8.794) \\
0.440\end{array}$ & $\begin{array}{l}(13.13) \\
2.353\end{array}$ & $(9.343)$ & $(9.414)$ \\
\hline EMF & & $\begin{array}{l}1.973 \\
(9.453)\end{array}$ & $\begin{array}{l}0.397 \\
(.698)\end{array}$ & $\begin{array}{l}1.230 \\
(9.568)\end{array}$ & $\begin{array}{l}1.905 \\
(7.448)\end{array}$ & $\begin{array}{l}-0.677 \\
(7.605)\end{array}$ & $\begin{array}{l}0.502 \\
(7.065)\end{array}$ & $\begin{array}{l}0.440 \\
(8.937)\end{array}$ & $\begin{array}{c}2.353 \\
(13.35)\end{array}$ & $\begin{array}{l}1.642 \\
(9.516)\end{array}$ & $\begin{array}{l}0.106 \\
(9.521)\end{array}$ \\
\hline PR5 & & $-35.92^{* * * *}$ & $-11.43^{*}$ & $\begin{array}{l}-36.20^{* * * *} \\
\end{array}$ & -5.909 & -6.129 & -7.515 & -14.07 & $-52.60 * * *$ & $-35.95^{* * *}$ & $\begin{array}{l}(9.021) * * \\
-35.93^{* * *}\end{array}$ \\
\hline & & $(9.323)$ & $(6.616)$ & $(9.444)$ & $(7.363)$ & (7.533) & $(6.982)$ & $(8.797)$ & $(13.19)$ & $(9.369)$ & $(9.437)$ \\
\hline Constant & $\begin{array}{c}-2.708^{* * *} \\
(0.909)\end{array}$ & $\begin{array}{c}-134.9^{* * * *} \\
(9.618)\end{array}$ & $\begin{array}{c}-65.43^{* * * *} \\
(6.813)\end{array}$ & $\begin{array}{c}-129.4^{* * * *} \\
(9.752)\end{array}$ & 年 & - & $\begin{array}{c}-28.96^{* * * *} \\
(7.217)\end{array}$ & $\begin{array}{c}-95.72^{* * *} \\
(9.078)\end{array}$ & $\begin{array}{c}-173.0^{* * *} \\
(13.64)\end{array}$ & $\begin{array}{l}-1.457 \\
(1.329)\end{array}$ & $\begin{array}{l}-1.559 \\
(1.331)\end{array}$ \\
\hline LAG & NO & & & YES & YES & YES & YES & YES & $\mathrm{YE}$ & $\mathrm{YE}$ & YES \\
\hline Observations & 12,407 & 12,407 & 9,864 & 12,200 & 2,521 & 2,520 & 5,041 & 4,661 & 7,159 & 12,185 & 12,195 \\
\hline Countries & 5 & 5 & 4 & 5 & 1 & 1 & 2 & 2 & 3 & 5 & 5 \\
\hline R-squared & 0.018 & 0.028 & 0.092 & 0.033 & 0.219 & 0.220 & 0.200 & 0.131 & 0.048 & 0.025 & 0.023 \\
\hline
\end{tabular}

***, ${ }^{* *},{ }^{*}$ : significance at the 1 percent, 5 percent and 10 percent levels.

Notes: See Notes to Table 5 . 
Table C.2: Robustness exercise. 1-day window for the event analysis. 10-year spread with respect to the German reference.

\begin{tabular}{|c|c|c|c|c|c|c|c|c|c|c|c|}
\hline VARIABLES & (1) & (2) & (3) & (4) & (5) & (6) & (7) & (8) & (9) & (10) & (11) \\
\hline Decision $t-1$ & $\begin{array}{l}-1.426 \\
(1.684)\end{array}$ & $\begin{array}{l}-1.026 \\
(1.642)\end{array}$ & $\begin{array}{l}-0.240 \\
(1.133)\end{array}$ & $\begin{array}{l}-0.931 \\
(1.695)\end{array}$ & $\begin{array}{l}-0.694 \\
(1.334)\end{array}$ & $\begin{array}{l}0.419 \\
(1.365)\end{array}$ & $\begin{array}{l}0.0849 \\
(1.270)\end{array}$ & $\begin{array}{r}-1.619 \\
(1.573)\end{array}$ & $\begin{array}{r}-2.623 \\
(2.398)\end{array}$ & $\begin{array}{l}-1.223 \\
(1.832)\end{array}$ & $\begin{array}{l}-0.608 \\
-1.448\end{array}$ \\
\hline Decision $\mathrm{t}$ & -0.535 & $3.485^{* *}$ & $4.121^{* * *}$ & $3.305 * *$ & $3.246 * *$ & $3.091^{* *}$ & $3.205^{* *}$ & $4.769^{* * *}$ & $\begin{array}{l}(2.398) \\
2.406\end{array}$ & $\begin{array}{l}(1.832) \\
3.387^{*}\end{array}$ & $\begin{array}{l}(1.448) \\
3.004 * *\end{array}$ \\
\hline & $(1.681)$ & $(1.640)$ & $\begin{array}{l}(1.133) \\
\text { (1) }\end{array}$ & $(1.670)$ & $(1.308)$ & $(1.347)$ & $(1.248)$ & $(1.546)$ & $(2.366)$ & $(1.800)$ & $(1.431)$ \\
\hline Decision $t+1$ & $-4.442 * * *$ & $-4.690 * * *$ & $-3.306 * * *$ & $-4.655 * * *$ & $-2.653^{* *}$ & -1.718 & $-2.163^{*}$ & $-5.607^{* * * *}$ & $-6.628 * * *$ & $-3.813 * *$ & $-3.013^{* * *}$ \\
\hline & $(1.678)$ & $(1.625)$ & $(1.121)$ & $(1.653)$ & $(1.298)$ & $(1.332)$ & $(1.239)$ & $(1.535)$ & (2.337) & $(1.802)$ & (1.418) \\
\hline BIDASK & $\begin{array}{c}0.323 \\
(2.159)\end{array}$ & $\begin{array}{c}-3.020^{* * *} \\
(0.670)\end{array}$ & $\begin{array}{c}0.678 \\
(1.035)\end{array}$ & $\begin{array}{c}-3.060 * * * \\
(0.691)\end{array}$ & $\begin{array}{c}3.076 \\
(8.834)\end{array}$ & $\begin{array}{l}-4.984 \\
(15.68)\end{array}$ & $\begin{array}{l}-0.299 \\
(9.278)\end{array}$ & $\begin{array}{l}-0.557 \\
(1.105)\end{array}$ & $\begin{array}{c}-3.615^{* * *} \\
(0.705)\end{array}$ & $\begin{array}{c}-3.155^{* * *} \\
(0.678)\end{array}$ & $-3.079^{* * * *}$ \\
\hline$\triangle$ EONIA & $-2.750^{* * * *}$ & 0.610 & 2.159 & -0.0689 & $\begin{array}{l}(8.034) \\
-0.741\end{array}$ & $\begin{array}{l}-1.730 \\
\end{array}$ & -1.314 & $5.315^{*}$ & -0.586 & 1.284 & -0.103 \\
\hline & $(0.676)$ & $(2.900)$ & $(2.000)$ & $(2.938)$ & $(2.364)$ & $(2.429)$ & $(2.254)$ & $(2.756)$ & $(4.127)$ & $(2.932)$ & $(2.935)$ \\
\hline VIX & $\begin{array}{c}0.438 \\
(3.000)\end{array}$ & $\begin{array}{l}0.183^{3 * *} \\
(0.0425)\end{array}$ & $\begin{array}{l}0.0121 \\
(0.0301)\end{array}$ & $\begin{array}{l}0.199^{* * *} \\
(0.0445)\end{array}$ & $\begin{array}{l}-0.0378 \\
(0.0321)\end{array}$ & $\begin{array}{l}-0.0349 \\
(0.0322)\end{array}$ & $\begin{array}{l}-0.0272 \\
(0.0304)\end{array}$ & $\begin{array}{l}-0.0125 \\
(0.0393)\end{array}$ & $\begin{array}{l}0.312^{2 * * *} \\
(0.0644)\end{array}$ & $\begin{array}{l}0.203^{3 * * *} \\
(0.0443)\end{array}$ & $\begin{array}{l}0.195^{* * * *} \\
(0.0443)\end{array}$ \\
\hline $\mathrm{ECB}$ & $\begin{array}{l}0.2188^{* * *} \\
(0.0361)\end{array}$ & $\begin{array}{c}0.000133 \\
(1.049)\end{array}$ & $\begin{array}{c}-1.306^{*} \\
(0.719)\end{array}$ & $\begin{array}{l}-0.0413 \\
(1.069)\end{array}$ & $\begin{array}{c}-1.606^{*} \\
(0.839)\end{array}$ & $\begin{array}{c}-2.382^{* * *} \\
(0.859)\end{array}$ & $\begin{array}{c}-2.046^{* *} \\
(0.800)\end{array}$ & $\begin{array}{c}-0.419 \\
(1.001)\end{array}$ & $\begin{array}{c}1.682 \\
1.682 \\
(1.517)\end{array}$ & $\begin{array}{l}-0.0253 \\
(1.063)\end{array}$ & $\begin{array}{l}-0.0818 \\
(1.068)\end{array}$ \\
\hline$\triangle \mathrm{STOCK}$ & 1.216 & $-0.0211 * * *$ & $-0.0163^{* * *}$ & $-0.0207^{* * *}$ & $-0.0258^{* * *}$ & $-0.0130 * * *$ & $-0.0149 * * *$ & $-0.0663^{* * *}$ & $-0.0742 * * *$ & $-0.0216^{* * *}$ & $-0.0215^{* * *}$ \\
\hline MOODY & & & $(0.000561)$ & $(0.000842)$ & $(0.00125)$ & $(0.000573)$ & $(0.000631)$ & $(0.00316)$ & $(0.00450)$ & $(0.000828)$ & $(0.000829)$ \\
\hline MOODY & $\begin{array}{c}-0.0240 * * * \\
(0.000827)\end{array}$ & $\begin{array}{l}-0.551 \\
(3.121)\end{array}$ & $\begin{array}{c}-1.492 \\
(1.792)\end{array}$ & $\begin{array}{l}-2.896 \\
(3.109)\end{array}$ & $\begin{array}{r}2.689 \\
(2879)\end{array}$ & 0.535 & 1.699 & -2.397 & -4.812 & -2.914 & -2.951 \\
\hline DEFICIT & $\begin{array}{l}-0.274 \\
-10.03\end{array}$ & 0.750 & $\begin{array}{l}(1.192) \\
-0.234\end{array}$ & 1.082 & $\begin{array}{c}(2.872) \\
1.238\end{array}$ & $\begin{array}{c}(3.893) \\
1.506\end{array}$ & $\begin{array}{c}(2.483) \\
1.510\end{array}$ & $\begin{array}{l}(2.551) \\
-2.787\end{array}$ & $\begin{array}{c}(4.112) \\
0.226\end{array}$ & $\begin{array}{c}(3.048) \\
0.708\end{array}$ & $\begin{array}{l}(3.058) \\
0.617\end{array}$ \\
\hline & $(3.176)$ & $(2.238)$ & $(1.555)$ & $(2.275)$ & $(1.806)$ & (1.849) & $(1.721)$ & $(2.131)$ & $(3.225)$ & $(2.254)$ & $(2.267)$ \\
\hline BAILOUT & & $\begin{array}{l}-1.522 \\
(3089)\end{array}$ & $\begin{array}{l}-0.553 \\
(2083 \\
\end{array}$ & $\begin{array}{r}-1.663 \\
(3.137\end{array}$ & 0.645 & $\begin{array}{l}1.902 \\
(2.458)\end{array}$ & 1.615 & $\begin{array}{l}-4.542 \\
(3.008)\end{array}$ & -4.971 & -1.716 & -1.679 \\
\hline WIT & & $\begin{array}{r}(36.089) * * \\
-36.06 * *\end{array}$ & $\begin{array}{r}(24.083 * * * * \\
-24.58 *\end{array}$ & $\begin{array}{r}(35.137 * * * \\
-33^{* * *}\end{array}$ & $\begin{aligned} &(2.398) \\
&-47.04^{* * * *}\end{aligned}$ & $\begin{array}{r}(2.458) \\
-34.37^{* * *}\end{array}$ & $\begin{aligned} &(2.287) \\
&-42.05^{* * *}\end{aligned}$ & $\begin{array}{c}(3.008) \\
6.461\end{array}$ & $\begin{array}{l}(4.614) \\
-29.42^{*}\end{array}$ & $\begin{array}{c}(3.119) \\
-35.09^{* * *}\end{array}$ & $\begin{array}{c}(3.133) \\
-35.21 * * *\end{array}$ \\
\hline & & $(10.45)$ & $(6.553)$ & $(10.58)$ & $(7.607)$ & $(7.762)$ & $(7.236)$ & $(10.93)$ & $(17.46)$ & $(10.52)$ & $(10.57)$ \\
\hline WIT period & & $\begin{array}{c}-1.946 * * * \\
(0.651)\end{array}$ & $\begin{array}{c}-1.461 * * * \\
(0.460)\end{array}$ & $\begin{array}{c}-1.944^{* * * *} \\
(0.683)\end{array}$ & $\begin{array}{c}-1.194^{* *} \\
(0.492)\end{array}$ & $\begin{array}{c}-1.060 * * \\
(0.525)\end{array}$ & $\begin{array}{c}-1.115^{* *} \\
(0.470)\end{array}$ & $-1.745^{* * * *}$ & $-2.674^{* * *}$ & $-2.017^{* * * *}$ & $-1.949 * * *$ \\
\hline APP & & $-18.44^{* *}$ & -4.031 & $-17.93^{*}$ & -3.446 & 0.123 & -2.123 & $\begin{array}{l}(0.0318 \\
-3.518\end{array}$ & $\begin{array}{l}(1.020 * \\
-27.27^{* *}\end{array}$ & $-17.90^{*}$ & $-17.83^{*}$ \\
\hline & & $(9.399)$ & $(6.520)$ & $(9.546)$ & $(7.591)$ & $(7.772)$ & $(7.240)$ & $(8.778)$ & $(13.26)$ & $(9.488)$ & $(9.536)$ \\
\hline APP period & & $\begin{array}{l}-1.329^{* *} \\
(0.577)\end{array}$ & $-0.883^{* *}$ & $-1.296^{* *}$ & $-0.900^{*}$ & $-0.801 *$ & $-0.820^{*}$ & $-1.294^{* *}$ & $-1.874^{* *}$ & $-1.365^{* *}$ & $-1.291 * *$ \\
\hline & & $(0.577)$ & $(0.412)$ & $(0.605)$ & $(0.463)$ & $(0.481)$ & $(0.435)$ & $(0.613)$ & $(0.911)$ & $(0.601)$ & $(0.600)$ \\
\hline Two pack & & $\begin{array}{l}-18.82^{*} \\
(10.44)\end{array}$ & $\begin{array}{l}-7.370 \\
(6.552)\end{array}$ & $\begin{array}{c}-24.02^{* * *} \\
(10.57)\end{array}$ & $\begin{array}{l}-11.95 \\
(7.574)\end{array}$ & $\begin{array}{l}3.488 \\
(7.747)\end{array}$ & $\begin{array}{l}-3.843 \\
(7.219)\end{array}$ & $\begin{array}{l}-12.84 \\
(10.92)\end{array}$ & $\begin{array}{c}-53.76^{\text {*** }} \\
(17.47)\end{array}$ & $\begin{array}{c}-24.24^{* *} \\
(10.52)\end{array}$ & $\begin{array}{c}-23.12^{* * *} \\
(10.56)\end{array}$ \\
\hline SSM & & $-20.95^{* *}$ & $-26.73 * * *$ & $-20.25^{*}$ & $-40.99^{* * *}$ & $-23.29^{* * *}$ & $-33.35 * * *$ & $\begin{array}{l}(10.92) \\
-12.31\end{array}$ & $\begin{array}{l}(17.47) \\
-4.294\end{array}$ & $\begin{array}{l}(10.52) \\
-19.98^{*}\end{array}$ & $\begin{array}{l}(10.56) \\
-19.98^{*}\end{array}$ \\
\hline & & $(10.46)$ & $(6.575)$ & $(10.61)$ & $(7.597)$ & $(7.779)$ & $(7.237)$ & (10.93) & $(17.55)$ & $(10.56)$ & $\begin{array}{l}-19.98^{*} \\
(10.60)\end{array}$ \\
\hline $\mathrm{BICC}$ & & -9.040 & -8.634 & -10.33 & -9.404 & $-16.71^{* *}$ & $-12.06^{*}$ & -7.009 & -8.783 & -10.40 & -10.31 \\
\hline & & (9.351) & $(6.487)$ & $(9.497)$ & $(7.561)$ & (7.778) & $(7.217)$ & $(8.728)$ & (13.18) & $(9.440)$ & $(9.487)$ \\
\hline SRM & & $15.60 *$ & 8.343 & $15.73^{*}$ & $14.90^{* *}$ & 12.05 & $13.60^{*}$ & 4.840 & 16.80 & $15.74^{*}$ & $15.73^{*}$ \\
\hline & & (9.353) & (6.488) & $(9.497)$ & $(7.561)$ & $(7.734)$ & $(7.208)$ & $(8.731)$ & (13.19) & $(9.441)$ & $(9.488)$ \\
\hline EDIS & & 3.423 & -0.720 & 3.533 & -1.168 & -4.370 & -2.776 & 0.600 & 8.573 & 3.484 & 3.443 \\
\hline 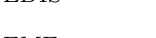 & & $(9.351)$ & $(6.486)$ & $(9.496)$ & (7.553) & $(7.731)$ & $(7.204)$ & $(8.726)$ & (13.18) & (9.439) & $(9.486)$ \\
\hline EMF & & 3.884 & 2.187 & 3.062 & 3.656 & 1.001 & 2.224 & 2.201 & 4.182 & 3.310 & 2.695 \\
\hline & & $(9.493)$ & $(6.584)$ & $(9.644)$ & $(7.672)$ & (7.853) & $(7.316)$ & (8.868) & $(13.40)$ & $(9.613)$ & $(9.594)$ \\
\hline PR5 & & $-27.94 * * *$ & $\begin{array}{l}-3.380 \\
6510\end{array}$ & $\begin{array}{c}-28.08^{* * * *} \\
(9.528)\end{array}$ & $\begin{array}{l}1.330 \\
(7.579)\end{array}$ & $\begin{array}{l}0.921 \\
(7.768)\end{array}$ & $\begin{array}{l}-0.312 \\
(7.224)\end{array}$ & $\begin{array}{r}-6.677 \\
(8.728)\end{array}$ & $\begin{array}{c}-44.24^{* * *} \\
(13.25)\end{array}$ & $-27.88 * * *$ & $-27.88^{* * * *}$ \\
\hline Constant & $\begin{array}{c}-2.692 * * * \\
(0.919)\end{array}$ & $\begin{array}{c}-141.7^{* * * *} \\
(9.665)\end{array}$ & $\begin{array}{c}\left(72.97^{* * *}\right. \\
(6.708)\end{array}$ & $\begin{array}{c}(19.528) * \\
-13.5 * * * \\
(9.841)\end{array}$ & (7.579) & $\begin{array}{c}(7.768) \\
-\end{array}$ & $\begin{array}{c}(7.224) \\
-35.25 * * * \\
(7.468)\end{array}$ & $\begin{array}{c}(8.728) \\
-102.2^{* * *} \\
(9.009)\end{array}$ & $\begin{array}{c}(13.25) \\
-178.0^{* * *} \\
(13.71)\end{array}$ & $\begin{array}{l}(9.471) \\
-1.434 \\
(1.352)\end{array}$ & $\begin{array}{l}(9.515) \\
-1.534 \\
(1.352)\end{array}$ \\
\hline LAG & NO & NO & NO & YES & YES & YES & YES & YES & YES & YES & YES \\
\hline Observations & 12,384 & 12,384 & 9,844 & 12,187 & 2,519 & 2,516 & 5,035 & 4,655 & 7,152 & 12,172 & 12,182 \\
\hline $\begin{array}{l}\text { Countries } \\
\text { R-squared }\end{array}$ & $\begin{array}{c}5 \\
0.018\end{array}$ & $\begin{array}{c}5 \\
0.029\end{array}$ & $\begin{array}{c}4 \\
0.095\end{array}$ & $\begin{array}{c}5 \\
0.036\end{array}$ & $\begin{array}{c}1 \\
0.217\end{array}$ & $\begin{array}{c}1 \\
0.216\end{array}$ & $\begin{array}{c}2 \\
0.197\end{array}$ & $\begin{array}{c}2 \\
0.134\end{array}$ & $\begin{array}{c}3 \\
0.052\end{array}$ & $\begin{array}{c}5 \\
0.027\end{array}$ & $\begin{array}{c}5 \\
0.025\end{array}$ \\
\hline R-squared & 0.018 & & & & & 0.216 & & & 0.052 & & 0.025 \\
\hline
\end{tabular}

${ }^{* * *},{ }^{* *},{ }^{*}$ : significance at the 1 percent, 5 percent and 10 percent levels.

Notes: See Notes to Table 6 


\section{BANCO DE ESPAÑA PUBLICATIONS}

\section{WORKING PAPERS}

1940 MYROSLAV PIDKUYKO: Heterogeneous spillovers of housing credit policy.

1941 LAURA ÁLVAREZ ROMÁN and MIGUEL GARCÍA-POSADA GÓMEZ: Modelling regional housing prices in Spain.

1942 STÉPHANE DÉES and ALESSANDRO GALESI: The Global Financial Cycle and US monetary policy

in an interconnected world.

1943 ANDRÉS EROSA and BEATRIZ GONZÁLEZ: Taxation and the life cycle of firms.

1944 MARIO ALLOZA, JESÚS GONZALO and CARLOS SANZ: Dynamic effects of persistent shocks.

1945 PABLO DE ANDRÉS, RICARDO GIMENO and RUTH MATEOS DE CABO: The gender gap in bank credit access.

1946 IRMA ALONSO and LUIS MOLINA: The SHERLOC: an EWS-based index of vulnerability for emerging economies.

1947 GERGELY GANICS, BARBARA ROSSI and TATEVIK SEKHPOSYAN: From Fixed-event to Fixed-horizon Density Forecasts: Obtaining Measures of Multi-horizon Uncertainty from Survey Density Forecasts.

1948 GERGELY GANICS and FLORENS ODENDAHL: Bayesian VAR Forecasts, Survey Information and Structural Change in the Euro Area.

2001 JAVIER ANDRÉS, PABLO BURRIEL and WENYI SHEN: Debt sustainability and fiscal space in a heterogeneous Monetary Union: normal times vs the zero lower bound.

2002 JUAN S. MORA-SANGUINETTI and RICARDO PÉREZ-VALLS: ¿Cómo afecta la complejidad de la regulación a la demografía empresarial? Evidencia para España

2003 ALEJANDRO BUESA, FRANCISCO JAVIER POBLACIÓN GARCÍA and JAVIER TARANCÓN: Measuring the procyclicality of impairment accounting regimes: a comparison between IFRS 9 and US GAAP.

2004 HENRIQUE S. BASSO and JUAN F. JIMENO: From secular stagnation to robocalypse? Implications of demographic and technological changes.

2005 LEONARDO GAMBACORTA, SERGIO MAYORDOMO and JOSÉ MARÍA SERENA: Dollar borrowing, firm-characteristics, and FX-hedged funding opportunities.

2006 IRMA ALONSO ÁLVAREZ, VIRGINIA DI NINO and FABRIZIO VENDITTI: Strategic interactions and price dynamics in the global oil market.

2007 JORGE E. GALÁN: The benefits are at the tail: uncovering the impact of macroprudential policy on growth-at-risk.

2008 SVEN BLANK, MATHIAS HOFFMANN and MORITZ A. ROTH: Foreign direct investment and the equity home bias puzzle.

2009 AYMAN EL DAHRAWY SÁNCHEZ-ALBORNOZ and JACOPO TIMINI: Trade agreements and Latin American trade (creation and diversion) and welfare.

2010 ALFREDO GARCÍA-HIERNAUX, MARÍA T. GONZÁLEZ-PÉREZ and DAVID E. GUERRERO: Eurozone prices: a tale of convergence and divergence.

2011 ÁNGEL IVÁN MORENO BERNAL and CARLOS GONZÁLEZ PEDRAZ: Sentiment analysis of the Spanish Financial Stability Report. (There is a Spanish version of this edition with the same number).

2012 MARIAM CAMARERO, MARÍA DOLORES GADEA-RIVAS, ANA GÓMEZ-LOSCOS and CECILIO TAMARIT: External imbalances and recoveries.

2013 JESÚS FERNÁNDEZ-VILLAVERDE, SAMUEL HURTADO and GALO NUÑO: Financial frictions and the wealth distribution.

2014 RODRIGO BARBONE GONZALEZ, DMITRY KHAMETSHIN, JOSÉ-LUIS PEYDRÓ and ANDREA POLO: Hedger of last resort: evidence from Brazilian FX interventions, local credit, and global financial cycles.

2015 DANILO LEIVA-LEON, GABRIEL PEREZ-QUIROS and EYNO ROTS: Real-time weakness of the global economy: a first assessment of the coronavirus crisis.

2016 JAVIER ANDRÉS, ÓSCAR ARCE, JESÚS FERNÁNDEZ-VILLAVERDE and SAMUEL HURTADO: Deciphering the macroeconomic effects of internal devaluations in a monetary union.

2017 FERNANDO LÓPEZ-VICENTE, JACOPO TIMINI and NICOLA CORTINOVIS: Do trade agreements with labor provisions matter for emerging and developing economies' exports?

2018 EDDIE GERBA and DANILO LEIVA-LEON: Macro-financial interactions in a changing world.

2019 JAIME MARTÍNEZ-MARTÍN and ELENA RUSTICELLI: Keeping track of global trade in real time.

2020 VICTORIA IVASHINA, LUC LAEVEN and ENRIQUE MORAL-BENITO: Loan types and the bank lending channel.

2021 SERGIO MAYORDOMO, NICOLA PAVANINI and EMANUELE TARANTINO: The impact of alternative forms of bank consolidation on credit supply and financial stability.

2022 ALEX ARMAND, PEDRO CARNEIRO, FEDERICO TAGLIATI and YIMING XIA: Can subsidized employment tackle long-term unemployment? Experimental evidence from North Macedonia. 
2023 JACOPO TIMINI and FRANCESCA VIANI: A highway across the Atlantic? Trade and welfare effects of the EU-Mercosur agreement.

2024 CORINNA GHIRELLI, JAVIER J. PÉREZ and ALBERTO URTASUN: Economic policy uncertainty in Latin America: measurement using Spanish newspapers and economic spillovers.

2025 MAR DELGADO-TÉLLEZ, ESTHER GORDO, IVÁN KATARYNIUK and JAVIER J. PÉREZ: The decline in public investment: "social dominance" or too-rigid fiscal rules?

2026 ELVIRA PRADES-ILLANES and PATROCINIO TELLO-CASAS: Spanish regions in Global Value Chains: How important? How different?

2027 PABLO AGUILAR, CORINNA GHIRELLI, MATÍAS PACCE and ALBERTO URTASUN: Can news help measure economic sentiment? An application in COVID-19 times.

2028 EDUARDO GUTIÉRREZ, ENRIQUE MORAL-BENITO, DANIEL OTO-PERALÍAS and ROBERTO RAMOS: The spatial distribution of population in Spain: an anomaly in European perspective.

2029 PABLO BURRIEL, CRISTINA CHECHERITA-WESTPHAL, PASCAL JACQUINOT, MATTHIAS SCHÖN and NIKOLAI STÄHLER: Economic consequences of high public debt: evidence from three large scale DSGE models.

2030 BEATRIZ GONZÁLEZ: Macroeconomics, Firm Dynamics and IPOs.

2031 BRINDUSA ANGHEL, NÚRIA RODRÍGUEZ-PLANAS and ANNA SANZ-DE-GALDEANO: Gender Equality and the Math Gender Gap.

2032 ANDRÉS ALONSO and JOSÉ MANUEL CARBÓ: Machine learning in credit risk: measuring the dilemma between prediction and supervisory cost.

2033 PILAR GARCÍA-PEREA, AITOR LACUESTA and PAU ROLDAN-BLANCO: Raising Markups to Survive: Small Spanish Firms during the Great Recession.

2034 MÁXIMO CAMACHO, MATÍAS PACCE and GABRIEL PÉREZ-QUIRÓS: Spillover Effects in International Business Cycles.

2035 ÁNGEL IVÁN MORENO and TERESA CAMINERO: Application of text mining to the analysis of climate-related disclosures.

2036 EFFROSYNI ADAMOPOULOU and ERNESTO VILLANUEVA: Wage determination and the bite of collective contracts in Italy and Spain: evidence from the metal working industry.

2037 MIKEL BEDAYO, GABRIEL JIMÉNEZ, JOSÉ-LUIS PEYDRÓ and RAQUEL VEGAS: Screening and Loan Origination Time: Lending Standards, Loan Defaults and Bank Failures.

2038 BRINDUSA ANGHEL, PILAR CUADRADO and FEDERICO TAGLIATI: Why cognitive test scores of Spanish adults are so low? The role of schooling and socioeconomic background

2039 CHRISTOPH ALBERT, ANDREA CAGGESE and BEATRIZ GONZÁLEZ: The Short- and Long-run Employment Impact of COVID-19 through the Effects of Real and Financial Shocks on New Firms.

2040 GABRIEL JIMÉNEZ, DAVID MARTíNEZ-MIERA and JOSÉ-LUIS PEYDRÓ: Who Truly Bears (Bank) Taxes? Evidence from Only Shifting Statutory Incidence.

2041 FELIX HOLUB, LAURA HOSPIDO and ULRICH J. WAGNER: Urban air pollution and sick leaves: evidence from social security data.

2042 NÉLIDA DÍAZ SOBRINO, CORINNA GHIRELLI, SAMUEL HURTADO, JAVIER J. PÉREZ and ALBERTO URTASUN: The narrative about the economy as a shadow forecast: an analysis using Banco de España quarterly reports.

2043 NEZIH GUNER, JAVIER LÓPEZ-SEGOVIA and ROBERTO RAMOS: Reforming the individual income tax in Spain.

2101 DARÍO SERRANO-PUENTE: Optimal progressivity of personal income tax: a general equilibrium evaluation for Spain.

2102 SANDRA GARCÍA-URIBE, HANNES MUELLER and CARLOS SANZ: Economic uncertainty and divisive politics: evidence from the Dos Españas.

2103 IVÁN KATARYNIUK, VÍCTOR MORA-BAJÉN and JAVIER J. PÉREZ: EMU deepening and sovereign debt spreads: using political space to achieve policy space. 